Master thesis report

October 16, 2012

\title{
Assessing the MR compatibility of dental retainer wires at 7 tesla
}

Author:

Joep Wezel
Supervisors:

Dr. ir. B.J. Kooij ${ }^{1}$

Prof. dr. A.G. Webb ${ }^{2}$

Dr. ir. R. F. Remis ${ }^{1}$

\section{TUDelft}

${ }^{1}$ Microwave Technology and Systems for Radar Department of Electrical Engineering

Faculty of Electrical Engineering, Mathematics and Computer Science Delft University of Technology

\section{$\stackrel{\mathrm{LU}}{\mathrm{MC}}$}

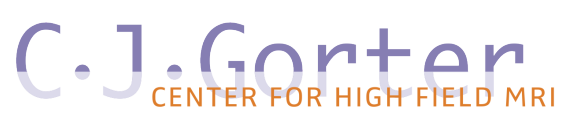

${ }^{2}$ C.J. Gorter Center for High Field Magnetic Resonance

Department of Radiology

Leiden University Medical Center 


\section{Thesis abstract}

The radio frequent $(\mathrm{RF})$ safety of dental retainer wires at 7 tesla has been investigated. Previous studies on metal implant wires at lower field strengths indicate possible SAR increase and directly related a temperature rise of the tissue around the wire. In the Netherlands up to $40 \%$ of the younger adults have such a dental retainer wire, which at the moment is listed as a contraindication for $7 \mathrm{~T}$ head scans.

The radio frequent $(\mathrm{RF})$ field transmitted by the MR excitation head coil is responsible for heating of the wire and its surroundings, of which the electric fields directly contribute to tissue heating. A range of retainer wire lengths of 0 to $70 \mathrm{~mm}$ have been tested in a gelled saline phantom in the 7T head coil. Temperature measurements on these wires followed the ASTM F2182-02a standard test method for measurement of radio frequency induced heating near passive implants. The temperature results after 15 minutes of excitation are compared to electromagnetic and temperature simulations. By confirming that phantom simulation and measurement results agree well and therefore are reliable we can make valid conclusions extending to a head model.

Head model electromagnetic simulations provided results in which a retainer wire of common wire length did not create a 1 gram SAR higher than than the maximum $1 \mathrm{~g}$ SAR present in the head without the wire. It could be argued that due to the fact that the wire is not creating the highest SAR, this wire can be rated MR compatible. However, the phantom study showed a 10 fold increase in the 1 gram SAR for a resonant wire. A long retainer wire in the head could become resonant and therefore unsafe, although depending on the surrounding tissues most of the common wire lengths are below resonance length. A solution to this problem has been investigated which resulted in an electrically non-conductive spacer around the wire that lowers the local SAR. The spacer, which is fully surrounding the wire, does not allow conduction currents, induced by the incident field of the MR head coil, to flow from the tissue towards the wire. An experimentally verified spacer with a layer thickness of 0.38 reduced the 1 gram SAR of a resonant wire by a factor of 8 . The head model with a $3 \mathrm{~mm}$ spacing between tongue and wire had no local SAR increase compared to the model without wire, making the retainer wire with spacer MR compatible.

Concluding, small retainer wires up to $32 \mathrm{~mm}$ of length are MR compatible for a head scan. Longer wires could be safe, depending on the tissues present around the wire. To be certain, the longer wires can be made safe when a non-conductive spacer is applied, covering the whole wire. A practical solution would be to use a silicone sports mouthguard. As a consequence of these outcomes, people with a retainer wire have the possibility of going into the 7 tesla MR scanner. 


\section{Preface}

The work for this thesis has been carried out at the site of the 7 tesla MR scanner of the C.J. Gorter center for high field MRI at the LUMC in Leiden. This exceptionally powerful scanner enables exploration of new areas of brain research through the highly detailed images. This provides for instance the possibility of identifying brain related diseases at an earlier stage compared to the conventional 1.5 or 3 tesla systems.

The thesis topic is assessing the safety of having a dental retainer wire in the 7 tesla scanner. These metal wires are accepted in the 1.5 and 3 tesla clinical systems, however, the wires are a contra-indication when undertaking a $7 \mathrm{~T}$ head scan. This difference is stemming from the fact that the higher RF frequency of the $7 \mathrm{~T}$ scanner could induce a resonant current in the metal wire. The contra-indication affects a large group of people. In the Netherlands up to $40 \%$ of the younger population around 21 years of age are carrying a retainer wire. The goal of this study is to assess the safety of the retainer wires and to find a solution when the level of safety is questioned such that the new $7 \mathrm{~T}$ scans are available to more people.

This work has been performed in the partial fulfillment of the requirements for the MSc degree in Electrical Engineering at the Technical University Delft.

\section{Acknowledgement}

I would like to thank the colleagues at the C.J. Gorter center for their enthusiasm, the fun we had while running, swimming and dancing and for explaining how MRI really works. Also for the MR related trips to the meetings in Germany and to the ISMRM BeNeLux meeting in Leuven, where I could present my preliminary work for the first time to a larger audience. I am very grateful to have been part of the global ISMRM conference in Australia, thanks to the guidance of Andrew Webb and with financial support from the Telecommunications department by Alexander Yarovoy and Bert Jan Kooij, the University Fund Delft and the Kivi Niria travel fund. Spending a full week together with young MR enthusiasts was inspiring.

I would like to thank my supervisor Andrew Webb at the Gorter Center for his advice from the start of the project and for welcoming me to the group. I am grateful to my supervisors in Delft, Bert Jan Kooij and Rob Remis for their theoretical input to the project. Also Paul de Heer and Wyger Brink for many helpful comments during the thesis. Furthermore a big thank you to my friends and family for their support around the deadlines. And especially to Josien for all her care and energy during the the last months of the thesis.

Joep Wezel

Amsterdam, October 2012 


\section{Notation and conventions}

\begin{tabular}{|c|c|c|c|}
\hline Symbol & Quantity name & Units & \\
\hline $\mathrm{S}$ & second & & \\
\hline $\mathrm{m}$ & meter & & \\
\hline V & volt & & \\
\hline A & ampere & & \\
\hline $\mathrm{C}$ & coulomb & & \\
\hline $\mathrm{T}$ & tesla & & \\
\hline $\mathrm{Hz}$ & herz & & \\
\hline $\mathrm{H}$ & henry & & \\
\hline $\mathrm{F}$ & farad & & \\
\hline $\mathbf{E}$ & Electric field strength & $\mathrm{V} / \mathrm{m}$ & \\
\hline $\mathbf{H}$ & Magnetic field strength & $\mathrm{A} / \mathrm{m}$ & \\
\hline $\mathbf{J}$ & Volume density electric current & $\mathrm{A} / \mathrm{m}^{2}$ & \\
\hline $\mathbf{K}$ & Volume density magnetic current & $\mathrm{V} / \mathrm{m}^{2}$ & \\
\hline $\mathbf{P}$ & Electric polarization & $\mathrm{C} / \mathrm{m}^{2}$ & \\
\hline M & Magnetization & $\mathrm{A} / \mathrm{m}$ & \\
\hline D & Electric flux density & $\mathrm{C} / \mathrm{m}^{2}$ & \\
\hline B & Magnetic flux density & $\mathrm{T}$ & \\
\hline$c_{0}$ & Speed of light in vacuum & $\mathrm{m} / \mathrm{s}$ & $299.79 \mathrm{~m} / \mathrm{s}$ \\
\hline$\epsilon_{0}$ & Permittivity of vacuum & $\mathrm{F} / \mathrm{m}$ & $8.85 \cdot 10^{-12} \mathrm{~F} / \mathrm{m}$ \\
\hline$\epsilon_{r}$ & relative permittivity & & dimensionless \\
\hline$h$ & Plank's constant & $J \cdot s$ & $6.63 \cdot 10^{-34} \mathrm{~J} \cdot \mathrm{s}$ \\
\hline$\gamma$ & Gyromagnetic ratio & $\mathrm{Hz} / \mathrm{T}$ & \\
\hline$k$ & Bolzmann constant & $\mathrm{J} / \mathrm{K}$ & $1.38 \cdot 10^{-23} \mathrm{~J} / \mathrm{K}$ \\
\hline$f$ & Frequency & $\mathrm{Hz}$ & \\
\hline$\lambda$ & Wavelength & $\mathrm{m}$ & \\
\hline$\mu_{0}$ & Permeability of vacuum & $\mathrm{H} / \mathrm{m}$ & $4 \pi \cdot 10^{-7} \mathrm{H} / \mathrm{m}$ \\
\hline$\mu_{r}$ & Relative permeability & & dimensionless \\
\hline$\sigma$ & Conductivity & $\mathrm{S} / \mathrm{m}$ & \\
\hline
\end{tabular}

MRI specific symbols:

\begin{tabular}{lll}
\hline$\alpha$ & Flip angle & degrees \\
$\boldsymbol{B}_{0}$ & Static main magnetic field & $\mathrm{T}$ \\
$\boldsymbol{B}_{1}^{+}, \boldsymbol{B}_{1}^{-}$ & RF circularly polarized magnetic fields & $\mathrm{T}$ \\
$\boldsymbol{M}_{0}$ & Initial magnetization vector & $\mathrm{A} / \mathrm{m}$ \\
$\mathrm{SAR}$ & Specific Absorption Rate & $\mathrm{W} / \mathrm{kg}$ \\
$\mathrm{T}_{1}$ & Spin-lattice relaxation time constant & $\mathrm{s}$ \\
$\mathrm{T}_{2}$ & Spin-spin relaxation time constant & $\mathrm{s}$
\end{tabular}




\section{Contents}

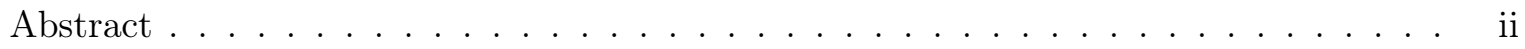

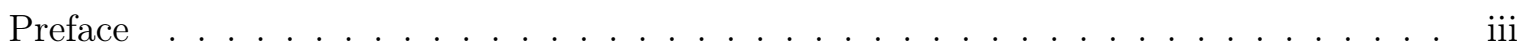

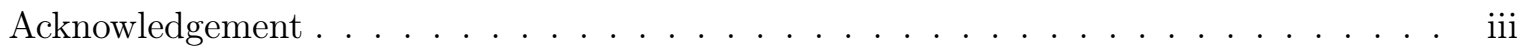

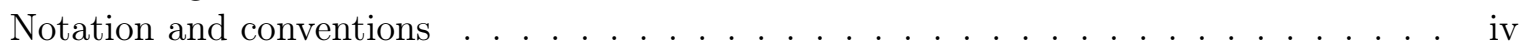

1 Introduction $\quad 1$

1.1 Problem statement . . . . . . . . . . . . . . . . . . . . . . 1

1.2 Project goal and approach . . . . . . . . . . . . . . . . . . . 1

2 MRI 3

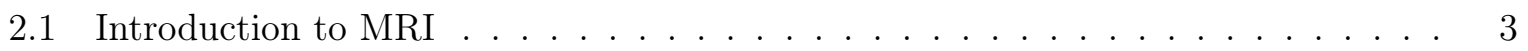

2.2 Imaging . . . . . . . . . . . . . . . . . . . . . . . . 9

2.3 MR Safety . . . . . . . . . . . . . . . . . . . . . . . . 10

2.3.1 Dissipated energy and Specific Absorption Rate . . . . . . . . . . . . . 11

3 Mathematical description of the RF field in MRI $\quad 13$

3.1 Basic electromagnetic equations . . . . . . . . . . . . . . . . 13

3.2 Electromagnetic field equations and constitutive equations . . . . . . . . . . . 13

3.3 The boundary conditions . . . . . . . . . . . . . . . . . . . . . 14

3.4 Time Fourier-transform domain field reciprocity theorem . . . . . . . . . . . 15

3.5 The electromagnetic RF field in the excitation state . . . . . . . . . . 17

3.6 The electromagnetic RF field in the receiving state . . . . . . . . . . . 20

4 Tissue heating around implants $\quad 23$

4.1 Cause of possible heating around a metal implant . . . . . . . . . . . . . 23

4.1.1 Temperature rise due to dissipated energy . . . . . . . . . . . . . 23

4.1.2 Electromagnetic resonators . . . . . . . . . . . . . . . . . 24

4.1.3 Previous studies on wire heating . . . . . . . . . . . 25

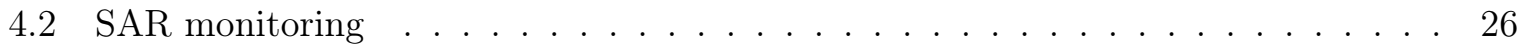

4.2 .1 SAR calorimetry . . . . . . . . . . . . . . . . 26

4.3 Spacer around the retainer wire for SAR reduction . . . . . . . . . . . . 27

4.4 SAR reduction around the wire . . . . . . . . . . . . . . . 27

4.4 Bare wire in tissue . . . . . . . . . . . . . . . . 28

4.4 Wire surrounded by spacer . . . . . . . . . . . . . . . 28

4.4 .3 Wire partially surrounded by spacer . . . . . . . . . . . . . . . . 29

4.4 .4 High E field in the spacer . . . . . . . . . . . . . . . . . . . . . . 29

4.5 Concluding remarks on tissue heating around a wire . . . . . . . . . 30

5 Temperature simulations and measurements 31

5.1 Magnetic deflection test . . . . . . . . . . . . . . . . . . . 31 
5.1.1 Magnetic deflection test setup . . . . . . . . . . . . . . . . . . . 31

5.1.2 Magnetic deflection test results and discussion . . . . . . . . . . . . . . 32

5.2 MRI simulations and measurements . . . . . . . . . . . . . . . . . . 32

5.2.1 Temperature measurements methods and test setup . . . . . . . . . . . 32

5.2 .2 Electromagnetic simulation methods . . . . . . . . . . . . . . . 34

5.2.3 Linking MR transmitted power to simulated transmitted power . . . . . . 34

$5.2 .4 \mathrm{~B}_{1}^{+}$Measurement and transmit efficiency . . . . . . . . . . . 35

5.3 Measurements and simulation results . . . . . . . . . . . . 36

5.3.1 Measured and simulated temperature rise: one wire . . . . . . . . . 36

5.3 .2 Measured and simulated temperature: wires 0 to $70 \mathrm{~mm} \ldots \ldots$. . . . . 38

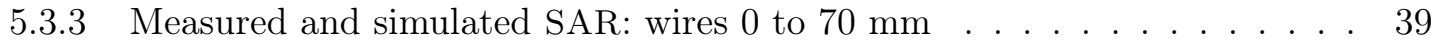

5.3 .4 Wire with spacer . . . . . . . . . . . . . . . . . . 41

5.3.5 Discussion on simulated and measured SAR and temperature rise . . . . . 41

6 EM simulations of phantom and head model in MRI birdcage 43

6.1 Electromagnetic simulation methods . . . . . . . . . . . . . 43

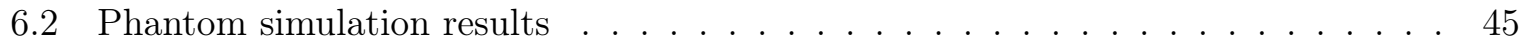

6.2 .1 SAR reduction and spacer thickness . . . . . . . . . . . . 45

6.2 .2 Spacer material selection . . . . . . . . . . . . . . . . . . . 46

6.2.3 Phantom simulations bended wire vs straight wire . . . . . . . . . . . 48

6.3 Head model simulations . . . . . . . . . . . . . . . . . . . . . . 49

6.4 Simulation data discussion and preliminary conclusions . . . . . . . . . . . 51

7 Discussion and conclusions $\quad 53$

7.1 Discussion . . . . . . . . . . . . . . . . . . . . 53

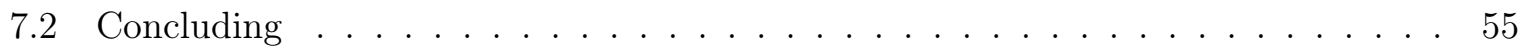

$\begin{array}{ll}\text { References } & 57\end{array}$ 


\section{Chapter 1}

\section{Introduction}

\subsection{Problem statement}

The safety of scanning a person with a retainer wire at 7 tesla is unclear in relation to the specific absorption rate (SAR ) increase around the wire and tissue heating. Previous studies on metal implant wires at lower field strengths indicate possible SAR increase and directly related a temperature rise of the tissue around the wire. Both could exceed the safety regulations. The safety limit on temperature rise for the head is $1^{\circ} \mathrm{C}$ and the SAR limit is $3.2 \mathrm{~W} / \mathrm{kg}$ whole head average, or $8 \mathrm{~W} / \mathrm{kg}$ for any 1 gram of tissue in the head as stated by the International Electrotechnical Commission (IEC).

Retainer wires are allowed at the lower field strength scanners of 1.5 and 3 tesla. The retainer wire is due to the RF safety issue a contra-indication at $7 \mathrm{~T}$ when performing a head scan.

\section{$1.2 \quad$ Project goal and approach}

Aim of the project is to shed light on the RF safety issues around heating of a dental retainer wire at $7 \mathrm{~T}$. This is done by quantifying the increase in tissue SAR caused by a metal retainer wire in the MR scanner.

Tissue SAR is the consequence of induced currents in the human body by the RF field. A wire will concentrate these currents and produce intense SAR at the tips. The current in the wire can be worsened for resonance conditions, amplifying the SAR at the tips. This resonance condition is variant to the surrounding tissue parameters, wire length and RF field operating frequency. The heterogeneous and time variant (tongue movement) nature of the retainer wire surroundings make the assessment of a worst case scenario not straight forward.

The safety of the retainer wire is assessed by numerical electromagnetic simulations both for a head model and for a phantom model. These simulations are validated by means of temperature measurements during SAR intense MR scans on a phantom with different lengths of wires. The temperature measurements are performed under the current guidelines of the ASTM standard for RF induced heating (F2182-11a). 


\section{Chapter 2}

\section{MRI}

This chapter includes an introduction to the extensive field of MRI. The principle of MRI is touched upon, followed by the main set of hardware components necessary for MR imaging. The introduction is concluded by a section on MR safety with a focus on RF induced temperature rise.

\section{$2.1 \quad$ Introduction to MRI}

Magnetic Resonance Imaging (MRI ) is being used in medical clinics with over 10 million scans prescribed annually (2010). Both analysis of shape anomalies in the human body and tissue identification is possible with MRI . The contrast in soft tissue images of the brain and other organs are unsurpassed by other imaging modalities. The image acquiring speed however is low compared to for example Computed Tomography (CT). The main hardware research trend in MRI is increasing the static magnetic field strength, this is beneficial for a multitude of parameters such as spatial resolution, signal to noise ratio and spectral resolution. The current clinical standard is the 3 tesla MR machine, however this study focusses on the stronger 7 tesla scanner which at the moment is still mainly used for research. The strength of the $7 \mathrm{~T}$ scanner is roughly 150,000 times greater than the Earth magnetic field strength which varies between $25 \mu T$ to $65 \mu T$. Numerous studies have investigated the safety of undergoing an MR scan, with respect to both the great strength of the static magnetic field and the use of strong radio frequent electromagnetic fields for imaging. Attention is paid especially to implanted metal devices. Comparing to the other imaging techniques of CT and X-ray does MR not show a detrimental effect on the health.

\section{MRI principle}

MRI is based on the spin property of protons in our body. The spin of a proton can be described as a spinning top, that is spinning around one axis and when placed in a static magnetic field like the earth magnetic field also starts rotating about a secondary axis. When a person is moved into an MR scanner, the proton spins will start rotating around the MRI 's strong static magnetic field. The spinning protons can be excited to a higher energy state via a radio frequency $(\mathrm{RF})$ circularly polarized clockwise oriented magnetic field $\mathrm{B}_{1}^{+}$, during which energy is stored. The energy is released as a secondary circularly polarized counterclockwise magnetic field $\mathrm{B}_{1}^{-}$again after the exciting RF field is turned off. The retransmitted magnetic field can be measured and 
provides the MR data. This in short is the concept of nuclear magnetic resonance NMR, which provides data for one sample of material. An image can be created when spatial encoding is applied, which is then called MRI . The following sections will elaborate on the details of MRI, starting with the hardware system. Then continuing on the more physics part of MRI : proton precession, proton resonance frequency and the intensity of the MRI signal coming from a large group protons.

\section{MRI hardware setup}

The MRI system in short contains the following hardware components, their use to the MR setup is explained in the following sections.

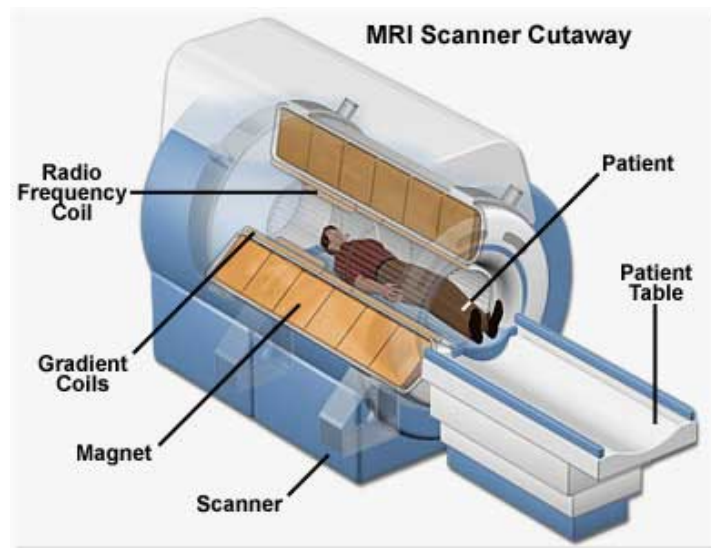

(a) MRI hardware

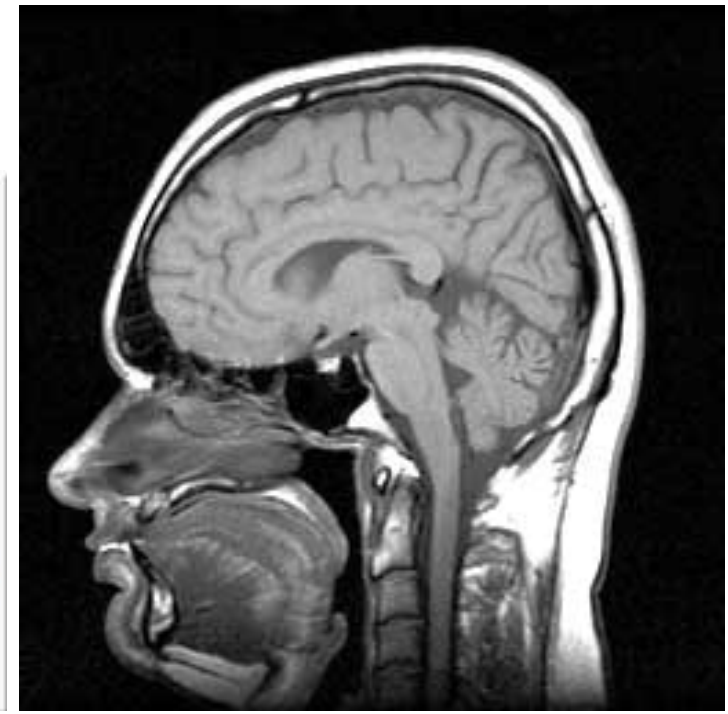

(b) Head scan

Figure 2.1: MRI hardware setup [2] and head scan [3]

The MRI scanner includes the following hardware components of which the main magnet is the most impressive. This powerful magnet creates a static magnetic field $\left(\boldsymbol{B}_{0}\right)$ which is pointing in the direction of the MR bore, by convention taken as the z-direction.

$$
\boldsymbol{B}_{0}=\mathrm{B}_{0} \boldsymbol{i}_{z}
$$

In which $\mathrm{B}_{0}$ is the magnitude of the field and $\boldsymbol{i}_{z}$ the unit vector pointing in z-direction. This field is created by a liquid hydrogen cooled super conducting magnet which is permanently in operation, day and night. This field has to be highly homogeneous in the scanner bore, a nonuniform $\boldsymbol{B}_{0}$ field will create a distorted image. Three set of gradient coils add a spatial varying magnetic field to the $\boldsymbol{B}_{0}$ field to encode spatial information. These gradient coils allow for image reconstruction. Excitation of the protons in the subject is achieved with an RF transmit antenna, commonly referred to as a coil in MRI literature. The retransmitted signal from the protons is received by either the same coil or by a separate one. The processing unit collects all data from the receive antenna after excitation which, combined with the gradient coil data, can reconstruct an MR image as depicted in Fig. 2.1b. 


\section{Proton spin}

MRI in a clinical setting is based on signals from protons in the human body. The proton rotates around its own internal axis with a given angular momentum $\mathbf{P}_{\text {ang }}$ and since the proton is a charged particle this creates a small magnetic moment $\boldsymbol{\mu}$,

$$
\left|\boldsymbol{\mu}_{\text {moment }}\right|=\gamma\left|\mathbf{P}_{\text {ang }}\right|
$$

The magnitude of the angular momentum is fixed and the magnitude of the magnetic moment is governed by the constant $\gamma$ which is the gyromagnetic ratio as described in eq. 2.1. The direction of this magnetic moment is random throughout the human body as depicted for hydrogen nuclei in Fig. 2.2a when the person is not in the MR environment. The sum of all magnetic moments is in this case zero. When placed in a strong magnetic field however, the alignment of the protons will result in a non-zero magnetization.

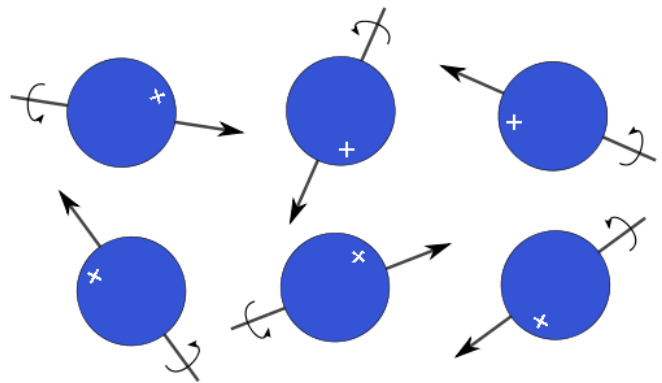

(a) Protons not aligned

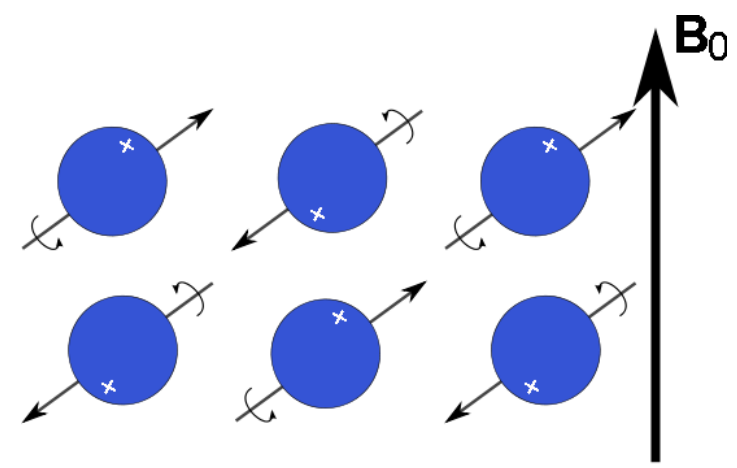

(b) Protons aligned to the $B_{0}$ field

Figure 2.2: Proton spin alignment when placed in the strong static magnetic field $B_{0}$

\section{Larmor resonance frequency}

The proton spins have a resonance frequency linearly related to the magnetic field strength $\boldsymbol{B}_{0}$ through the gyromagnetic constant. This special resonance frequency is the Larmor frequency named after the Irish physicist and mathematician Sir Joseph Larmor.

$$
\begin{aligned}
f_{\text {Larmor }} & =\bar{\gamma} \mathrm{B}_{0} \quad(H z) \\
f_{\text {hydrogen }} & =\bar{\gamma}_{\text {hydrogen }} \mathrm{B}_{0} \approx 42.58 \mathrm{~B}_{0} \quad(H z)
\end{aligned}
$$

$\bar{\gamma}$ is the gyromagnetic ratio in $\mathrm{Hz} / \mathrm{T}$ defining the ratio between magnetic field strength and the resonance frequency. The gyromagnetic ratio chosen in Eq. (2.3) is for hydrogen, which has $\bar{\gamma}_{\text {hydrogen }}=42.58 \cdot 10^{6} \mathrm{~Hz} / \mathrm{T}$. The resonant frequencies for hydrogen in a few common tissues at the standard clinical MR field strengths are given in the following table

Table 2.1: Larmor frequencies at the common MRI field strengths

\begin{tabular}{l|llll} 
Field strength $\mathrm{B}_{0}[\mathrm{~T}]$ & 1.5 & 3.0 & 7.0 & 9.4 \\
\hline Frequency $[\mathrm{MHz}]$ & 64 & 128 & 298.2 & 400
\end{tabular}

The main frequency for the 7 tesla scanner and consequently the frequency of interest in this study is $298.2 \mathrm{MHz}$. The trend in MRI is the increase in magnitude of the static magnetic 
field strength which is providing higher signal to noise ratio's of the received signals as will be described in the following section.

\section{MR sensitivity and energy state}

The sensitivity of the MR machine differs per chemical element for imaging such as Hydrogen, Fluor, Phosphor and Oxygen. Hydrogen returns the largest signal of all, both due to its large abundance[16] of $63 \%$ in the human body and its large gyromagnetic ratio. For tissue placed in the MR scanner, the magnetic moment of the spins align and start rotating about the direction of the static magnetic field $\boldsymbol{B}_{0}$ which is the z-direction. The angle between magnetic moment $\mu$ and the z-direction is fixed at 54.8 degrees either parallel or anti-parallel with the $\boldsymbol{B}_{0}$. The energy difference, $\Delta E$, between the two states is described by Eq. (2.4) where $h$ is Planck's constant $h=6.63 \cdot 10^{-34}[\mathrm{~J} \cdot \mathrm{s}]$.

$$
\begin{aligned}
& \Delta E=\Delta\left(E_{\text {anti-parallel }}, E_{\text {parallel }}\right)=\Delta\left(\frac{\gamma h \mathrm{~B}_{0}}{4 \pi},-\frac{\gamma h \mathrm{~B}_{0}}{4 \pi}\right) \\
& \Delta E=\frac{\gamma h B_{0}}{2 \pi} \quad(\mathrm{J})
\end{aligned}
$$

The ratio of protons in each state can be calculated using Boltzmann's equation in Eq. (2.5) in which an approximation for small exponent $\mathrm{x}$ can be made $e^{-x} \approx 1-x$. T is the temperature in the region of interest in Kelvin. It is noted that the parallel state is slightly more prevalent at room temperature.

$$
\frac{N_{\text {anti-parallel }}}{N_{\text {parallel }}}=e^{-\left[\frac{\Delta E}{k T}\right]}=e^{-\left[\frac{\gamma h \mathrm{~B}_{0}}{2 \pi k T}\right]} \approx 1-\left[\frac{\gamma h \mathrm{~B}_{0}}{2 \pi k T}\right]
$$

$N_{\text {total }}=$ Total number of protons in the region of interest and Bolzmann constant $k=1.38$. $10^{-23}[\mathrm{~J} / \mathrm{K}]$. The MR signal is related to difference of population sizes of the two energy states

$$
N_{\text {parallel }}-N_{\text {anti-parallel }}=N_{\text {total }} \frac{\gamma h \mathrm{~B}_{0}}{4 \pi k T}
$$

The additional number of protons in-parallel over the anti-parallel state depend on the $\mathrm{B}_{0}$ strength. As an example the difference in protons in the two states over the total number of protons can be calculated by equation 2.6. The difference in the head at $3 \mathrm{~T}$ for instance is only 10 protons more in parallel than for anti-parallel for a total of 1 million protons, which understandably leads to a small signal to pick up by the receive coil. The net magnetization in the body which the sum of the magnetic moments, see Fig. $2.2 \mathrm{~b}$ and 2.3 , is due to the parallel favoring state initially pointing in positive $\mathrm{z}$ direction. 


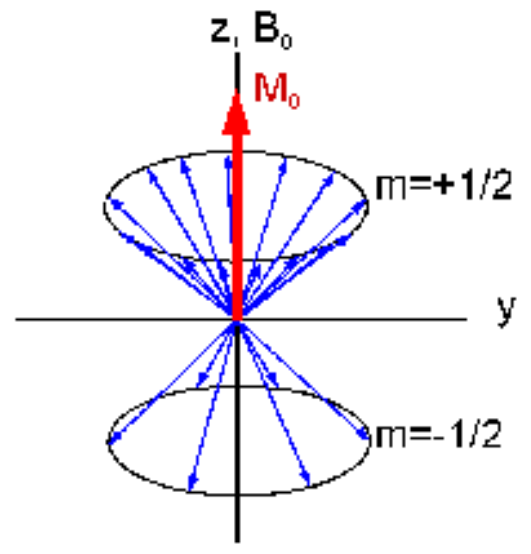

Figure 2.3: Net magnetization as function of the sum of magnetic moments

\section{Excitation state}

The magnetization vector in equilibrium is called $\boldsymbol{M}_{0}$ and is pointing in the $\boldsymbol{B}_{0}$ direction if no excitation is applied. When RF energy at the Larmor frequency is interacting with the proton spins this will flip them to a higher energy state. The transmitted magnetic field will effectively force the net magnetization to rotate from the equilibrium direction towards a desired flip angle. The magnetization vector will after excitation return to its equilibrium and retransmits a magnetic field during that process. The flip angle accounts amongst others to the strength of the received signal, it is therefore important to have equal flip angle excitation over the entire volume of interest.

The magnetic field created by the RF pulses is called the $\boldsymbol{B}_{1}$ field which has to be highly uniform in amplitude to obtain the same flip angles over the entire region of interest. In general the $\hat{\boldsymbol{B}}_{\mathbf{1}}$ in frequency domain is defined as

$$
\hat{\boldsymbol{B}}_{\mathbf{1}}=\hat{\mathrm{B}}_{1, x} \boldsymbol{i}_{x}+\hat{\mathrm{B}}_{1, y} \boldsymbol{i}_{y}+\hat{\mathrm{B}}_{1, z} \boldsymbol{i}_{z}
$$

The $\boldsymbol{B}_{1}$ field can be decomposed in three different components for which the effective $\hat{\boldsymbol{B}}_{\boldsymbol{1}}$ field for excitation is a left-handed rotating RF field in the rotation direction of the spins.

$$
\begin{aligned}
& \hat{\boldsymbol{B}}_{\mathbf{1}}=\hat{\boldsymbol{B}}_{1}^{+}+\hat{\boldsymbol{B}}_{1}^{-}+\hat{\mathrm{B}}_{1, z} \boldsymbol{i}_{z} \\
& \hat{\boldsymbol{B}}_{\mathbf{1}}=\hat{\mathrm{B}}_{1}^{+} \frac{\boldsymbol{i}_{x}+j \boldsymbol{i}_{y}}{\sqrt{2}}+\hat{\mathrm{B}}_{1}^{-} \frac{\boldsymbol{i}_{x}-j \boldsymbol{i}_{y}}{\sqrt{2}}+\hat{\mathrm{B}}_{1, z} \boldsymbol{i}_{z}
\end{aligned}
$$

The field magnitude of the left-handed circular RF field rotating around the z-axis of the MR coordinate system is termed the $\mathrm{B}_{1}^{+}$

$$
\mathrm{B}_{1}^{+}(\mathbf{x})=\frac{B_{1, x}(\mathbf{x})-j B_{1, y}(\mathbf{x})}{\sqrt{2}}
$$

A linearly polarized field will have a lower efficiency in tipping down the magnetization vector. A right-handed rotating field will have no effect at all on the flip angle. A birdcage antenna is usually chosen for excitation because its circular symmetry lends itself perfectly to create a 
symmetric rotating magnetic field. The flip angle reached is a function of pulse amplitude and excitation time length $\tau$

$$
\alpha(\mathbf{x})=\gamma \mathrm{B}_{1}^{+}(\mathbf{x}) \tau
$$

A good image requires equal flip angles at each location in the imaging region. A non uniform $\mathrm{B}_{1}^{+}$will create a distorted image because the flipangle distribution over the imaging region will not be uniform. An RF related engineering problem for higher field MR systems stems from the fact that the transmitted wavelength is in the same order of human body dimensions. Standing wave phenomena in the body are causing constructive and destructive interference, thereby creating dark and light spots in the acquired images.

The time $\tau$ it takes for the magnetization vector rotate to angle $\alpha$ is ideally instantaneous, though finite and bounded by the $\mathrm{B}_{1}^{+}$strength. High power RF pulses with peak power in the order of kilo watts are employed to reach the flip angle quickly.

\section{Receiving state}

After excitation of the magnetization vector up to an angle $\alpha$ the spins will start to return from the higher energy state towards their thermal equilibrium. Frequency and magnitude of the magnetization vector in the xy plane $\left(\boldsymbol{M}_{x y}\right)$ can be measured with pickup coils. This measurement is based on the fact that the magnetic flux through a coil induces an electric current. The magnetic field transmitted during relaxation produces a free induction decay (fid) signal at Larmor frequencies in the receive coils. The retransmitted field from the spins is rotating with reversed polarization $\mathrm{B}_{1}^{-}$as in Eq. (2.12) as opposed to the $\mathrm{B}_{1}^{+}$during excitation.

$$
\mathrm{B}_{1}^{-}(\mathbf{x})=\frac{B_{1, x}(\mathbf{x})+j B_{1, y}(\mathbf{x})}{\sqrt{2}}
$$

T1 relaxation The longitudinal magnetization $\boldsymbol{M}_{z}$, along the $\mathrm{z}$ axis, is reduced during $e x-$ citation by flipping the spins. The magnetization is returning to thermal equilibrium after excitation, back to $\boldsymbol{M}_{0}$. The recovery of the magnetization $\boldsymbol{M}_{z}$ back to $\boldsymbol{M}_{0}$ is following an exponential curve. The time it takes $M_{z}$ to reach $63 \%$ of the $\left(\left|\boldsymbol{M}_{0}\right|\right)$ is the $\mathrm{T}_{1}$.

T2 relaxation The magnitude of the transverse magnetization, $\boldsymbol{M}_{x y}$ is a summation of multiple spins whom initially after excitation are rotating having equal phase adding constructively to a large xy magnetization. These coherent phases are however spreading over time due to interaction between spins causing them to dephase. The time it takes for the xy-magnetization to decay to $37 \%$ of the initial signal is called $\mathrm{T}_{2}$-relaxation. The $\mathrm{T}_{2}$-relaxation is also called spin-spin relaxation. Both time constants $\mathrm{T}_{1}$ and $\mathrm{T}_{2}$ are tissue specific and are in the order of ms to seconds, in which the $T_{2}$ is always shorter than the $T_{1}$.

Reception of the MR signal can be performed with the same RF coil for excitation, although using different coils for excitation and reception are also possible. When the same coil for transmission/reception is used, care is taken for the receive circuitry since the output peak powers during excitation are in the order of kilo watts where as the receive circuitry has to be very sensitive to receive the low power $\mathrm{B}_{1}^{-}$field. For small signal detection surface coils for reception are often used, they are placed close to the skin to have a higher sensitivity. A mathematical description of the principle of $\mathrm{RF}$ fields in $\mathrm{MR}$ is covered in the next chapter (3). 


\subsection{Imaging}

Over the years sets of gradient coils were incorporated into the Nuclear Magnetic Resonance (NMR) setup to encode spatial information enabling MR imaging. Three sets of gradient coils add linear spatially varying magnetic fields on top of the $\boldsymbol{B}_{0}$ field making three dimensional imaging possible. A spatially varying non-RF magnetic gradient field, $\left(\mathbf{G}(x, y, z)=x G_{x}+\right.$ $\left.y G_{y}+z G_{z}\right) \boldsymbol{i}_{z}$ is applied over the body as shown in the following figure
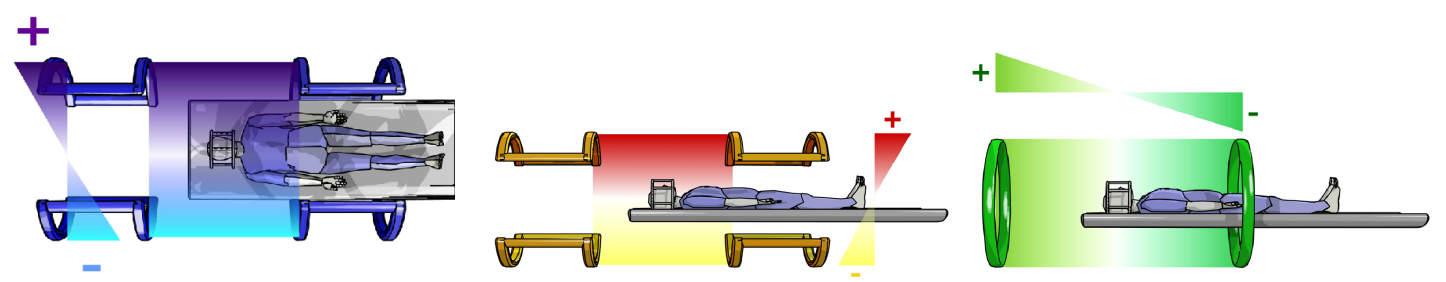

Figure 2.4: Gradient coils for spatial encoding in $\mathrm{x}, \mathrm{y}$ and $\mathrm{z}$ direction [27]

The gradient slope is desired to be as constant as possible e.g. to obtain a linearly varying field.

\section{Slice selection}

Imaging in clinical MRI is typically performed in slices (or slabs) in $\mathrm{x}, \mathrm{y}$ or $\mathrm{z}$ direction. The strength of the gradient field changes in the direction of the consecutive slices, in z direction for example as shown in the following figures

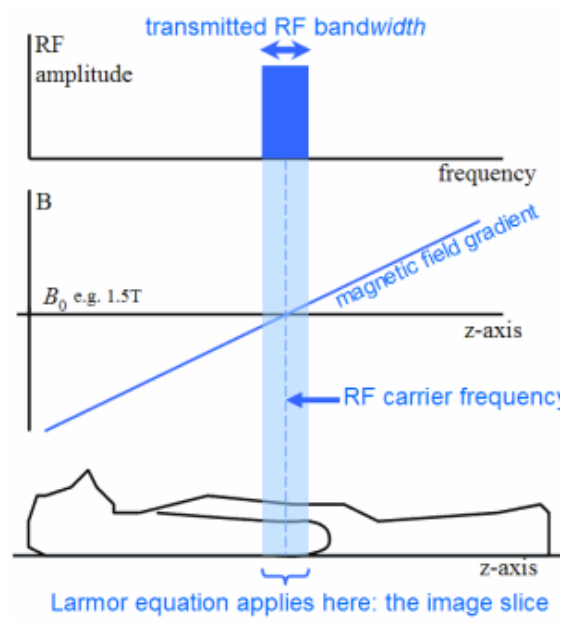

Figure 2.5: Slice selection through frequency selective pulse and gradient field

One slab is selected, as depicted in Fig. 2.5, by exciting the body with a frequency selective pulse of limited bandwidth. Only the protons spins with corresponding Larmor frequency are excited $\omega=\gamma\left(\left|\boldsymbol{B}_{0}\right|+\mathrm{zG}_{z}\right)$. The slab thickness is defined by the pulse bandwidth and gradient strength. The slice can be made thinner by either decreasing the pulse bandwidth or increasing the steepness of the slice selective gradient field. 


\section{Phase and frequency encoding}

The fid signal received from the sample after excitation contains signal contributions from all spins in the selected slice if the the other two gradients are off. The signal from the sample is picked up by receive coils, in which the signal is proportional to the proton density [28]. For the moment we consider the receive profile of the antenna $\left(b_{1}^{r e c}\right)$ to be constant over the entire imaging volume and assume the $\mathrm{T}_{2}$ to be spatially constant over the slice as well. Without xy-encoding we can write in general for the received signal from the selected slice in z-direction

$$
S\left(z_{\text {slice }}, t\right) \approx C e^{\frac{-t}{T_{2}}} \omega_{0}^{2} b_{1}^{r e c} e^{j \omega_{0} t} e^{j t \gamma G_{z} z_{\text {slice }}} \Delta z_{\text {slice }} \iint_{\text {slice }} \rho\left(x, y, z_{\text {slice }}\right) \mathrm{d} x \mathrm{~d} y
$$

When xy-encoding is applied, we obtain $k_{x}=-\mathrm{t} \gamma G_{x}$ and $\left.k_{y}=-\mathrm{t} \gamma G_{x}\right)$

$$
S\left(k_{x}, k_{y}, z_{\text {slice }}, t\right) \approx C e^{\frac{-t}{T_{2}}} \omega_{0}^{2} b_{1}^{r e c} e^{j \omega_{0} t} e^{j t \gamma G_{z} z_{\text {slice }}} \Delta z_{\text {slice }} \iint_{\text {slice }} \rho\left(x, y, z_{\text {slice }}\right) e^{-j x k_{x}-j y k_{y}} \mathrm{~d} x \mathrm{~d} y
$$

In which the received signal $\mathrm{S}$, which is defined in $\boldsymbol{k}$-space, is the 2D Fourier transform of the proton densities in the slice. Due to the assumption that $\mathrm{T}_{2}$ and the receive profile of the receive antenna are constant over all locations in the slice, we can write the integral purely over the proton densities in the xy plane. The $\mathrm{x}$ and $\mathrm{y}$ gradients are the frequency encoding and phase encoding gradient coils applying a linear varying field as depicted in Fig 2.4. A full xy-grid in the spatial frequency domain (k space) of received data points is build up step by step, line by line by cycling through the phase range and increasing the frequency gradient $G_{x}$ and $G_{y}$. In both equations of the received signal, Eqs. (2.13 and (2.14), we see that the Larmor frequency squared $\left(\omega_{0}^{2}\right)$ contributes to the power two to the received signal strength. A desire to move towards higher field strengths, thus higher Larmor frequency, is therefore explained since it provides an increase in received signal strength .

\section{$k$-space transformation}

The sampled full data space is in the spatial frequency domain ( $\boldsymbol{k}$-space). The MR image is obtained by taking the inverse Fourier transformation on this space. The resulting matrix is the MR image for example the head image such as depicted in Fig. 2.1b. The reconstructed image for a standard scan is a representation of the proton densities in the image field of view.

$$
\rho\left(x, y, z_{\text {slice }}\right) \approx \frac{1}{4 \pi^{2}} \frac{e^{\frac{t}{T_{2}}} e^{-j \omega_{0} t} e^{-j t \gamma G_{z} z_{\text {slice }}}}{C \omega_{0}^{2} b_{1}^{r e c} \Delta z_{\text {slice }}} \iint S\left(k_{x}, k_{y}, z_{\text {slice }}\right) e^{j\left(x k_{x}+y k_{y}\right)} \mathrm{d} k_{x} \mathrm{~d} k_{y}
$$

\section{$2.3 \quad$ MR Safety}

The transmitted pulsed RF peak power for excitation is in the order of $1 \mathrm{~kW}$ to $4 \mathrm{~kW}$. These high powers could lead to tissue heating and even irreversible tissue damage. To insure patient safety in the MR system, guidelines on the tissue Specific Absorption Rate (SAR) are in place. The SAR, which has units $\mathrm{W} / \mathrm{kg}$, is defined as the dissipated power in a $\mathrm{kg}$ of tissue and is derived from the dissipative term of the global form of the Poynting vector of energy balance as described in the following section. 


$$
\mathrm{SAR}=\frac{\sigma|\boldsymbol{E}|^{2}}{2 \rho}[W / k g]
$$

The SAR is directly linked to the dissipated power in a conductive material as described in the complex Poynting theorem in the following section

\subsubsection{Dissipated energy and Specific Absorption Rate}

The amount of electromagnetic power dissipated in tissue is described by the SAR in watt per kilogram of tissue. More generally, the time averaged amount of electromagnetic power flowing through a volume of matter is described by the Poynting vector $\boldsymbol{S}$, where brackets around $\langle\boldsymbol{S}\rangle$ indicate the time averaged power density of the electromagnetic fields in

$$
\langle\boldsymbol{S}\rangle=\frac{1}{2} \operatorname{Re}\left\{\boldsymbol{E} \times \boldsymbol{H}^{*}\right\} \quad W / m^{2}
$$

From this power density it can be seen that the direction of power is pointing orthogonal to both $\boldsymbol{E}$ and $\boldsymbol{H}$ fields. When we take the divergence of Poynting vector $\boldsymbol{S}$ [12] we obtain the electromagnetic power balance with the stored and dissipated EM energy. Specifically, for an isotropic material characterized by a scalar conductivity $\sigma$, permeability $\mu$ and permittivity $\epsilon$, we have

$$
\nabla \cdot\left(\boldsymbol{E} \times \boldsymbol{H}^{*}\right)=-j \omega \boldsymbol{H}^{*} \cdot \mu \boldsymbol{H}-j \omega \boldsymbol{E}^{*} \cdot \epsilon \boldsymbol{E}-\boldsymbol{E} \cdot \boldsymbol{J}^{*}
$$

This is known as the local complex Poyning theorem, without external sources. The three terms on the right side of the integral represent respectively the change in volume density of stored magnetic energy, the change in volume density of stored electric energy and the dissipated power density. This relation can be used to describe the power balance in the volume of a domain $\mathcal{D}$. The terms on both sides of the equal sign of Eq. 2.18 are integrated over the domain $\mathcal{D}$. This domain is bounded by the surface $\partial \mathcal{D}$. The power flowing out of this volume is defined by the Poynting vector in direction out of the volume orthogonal to $\partial \mathcal{D}$. Using Gauss' integral theorem also called the divergence theorem, we can write the divergence of the outer product $\left(\boldsymbol{E} \times \boldsymbol{H}^{*}\right)$ such that it represents the intantaneous power flowing from the volume $D$ to its surroundings over the surface of $\partial D$ in direction of $\boldsymbol{v}$ which is orthogonal to $\partial D$, as described in [13]

$$
\begin{aligned}
P^{\text {out }} & =\iiint_{\boldsymbol{x} \in \mathcal{D}} \boldsymbol{\nabla} \cdot(\boldsymbol{E} \times \boldsymbol{H}) d \mathrm{~V} \\
& =\iint_{\boldsymbol{x} \in \partial \mathcal{D}} \boldsymbol{v} \cdot(\boldsymbol{E} \times \boldsymbol{H}) \mathrm{d} A \\
& =\iint_{\boldsymbol{x} \in \partial \mathcal{D}} \boldsymbol{v} \cdot \boldsymbol{S} \mathrm{d} A
\end{aligned}
$$

We can write this amount of power flowing out of the volume in global form and by taking a lossy medium into account for which holds $\boldsymbol{J}=\sigma \boldsymbol{E}$, we obtain:

$$
\iint_{\boldsymbol{x} \in \partial \mathcal{D}} \boldsymbol{v} \cdot \boldsymbol{S} \mathrm{d} A=-\iiint_{\boldsymbol{x} \in \mathcal{D}} j \omega \boldsymbol{H}^{*} \mu \boldsymbol{H}+j \omega \boldsymbol{E}^{*} \epsilon \boldsymbol{E}+\boldsymbol{E} \cdot \sigma \boldsymbol{E}^{*} d \mathrm{~V}
$$

The third term in the volume integral describes the volume density of electromagnetic power that is irreversibly dissipated. The whole equation can be described, in the following way. The 
left side describes the out flowing power through the surface of $\partial \mathcal{D}$, the right side describes the change in amount of stored energy and secondly the dissipated in the volume $\mathcal{D}$. The sign in front of the the integral indicates that for a positive flow in direction of $\boldsymbol{v}$ the amount of energy in the domain decreases $\mathcal{D}$. When the dissipated power density is rewritten for a time average value, by taking the half of the real part to describe the volume density dissipated power as

$$
P_{\text {diss }}=\frac{1}{2} \sigma|\boldsymbol{E}|^{2} \quad W / m^{3},
$$

This describes the amount of power dissipated in a volume of conductive tissue as a function of the induced electric fields. From the point of thermal safety, energy dissipated per mass of tissue is more instructive than the energy in a volume since the density $(\rho)$ of tissue determines to a large extend how fast it will heat. The standards for RF safety therefore use the specific absorption rate

$$
\mathrm{SAR}=\frac{1}{2} \frac{\sigma|\boldsymbol{E}|^{2}}{\rho} W / k g
$$

This describes the amount of power dissipated per kilogram of tissue. Usually the averaged SAR over the whole body or body part is used. For smaller regions of interest generally a $10 \mathrm{~g}$ or $1 \mathrm{~g}$ SAR average is used, simulations allow for even smaller averages for local SAR in the order of milli grams.

Usually for a region of interest the highest maximum SAR is stated. The International Electrotechnical Commission (IEC) states a 1-gram average $\left(\mathrm{SAR}_{1 \mathrm{~g}}\right)$ of $8 \mathrm{~W} / \mathrm{kg}$ maximum for the head during 5 minutes, or a $3.2 \mathrm{~W} / \mathrm{kg}$ whole head average SAR during 10 minutes. The tissue close to an MR surface coil or close to a retainer wire will for instance have a local SAR much higher than the rest of the head. The 1 gram SAR average is more applicable than a whole head average, since the latter will underestimate the maximum amount of local RF energy absorbed. A local SAR (averaged over milligrams) around an implant however could overestimate the amount of energy absorbed. Since the field around an implant drops rather quickly, this region of high $\mathrm{SAR}_{\text {point }}$ is usually so small that the induced higher temperature is quickly distributed over a larger volume. The power transmitted during a SAR intense head scan is typically $20 \mathrm{~W}$ continuous, or lower. More on RF safety and heating in chapter. 4.

A second point of interest to safety is switching of the gradient coil during sequences, this switching can induce stimulations of the nervous system which is felt by twitching. The susceptibility to this stimulation differs per person. Reduction in slew rate of the gradient switching flanks can resolve the inconvenience. A third point of safety concern is the large attraction force which can be applied to metal objects such as implants. A large number of implants are made of titanium, those are not attracted by the main magnet. Some implants and retainer wires however are build of alloys containing the magnetic material nickel, the effect of the attraction force to the teeth will be noticable by the patient. Measurements of magnetic deflection of a dozen dental retainer wires in the scanner are documented in section 5.1. 


\section{Chapter 3}

\section{Mathematical description of the RF field in MRI}

This chapter describes the electromagnetic fields for the excitation and receiving state in a mathematical way. Lorentz reciprocity theorem is used to describe the interactions between the fields in the different states.

\subsection{Basic electromagnetic equations}

To specify position in the configuration, we employ the coordinates $\left\{x_{1}, x_{2}\right.$,

$\left.x_{3}\right\}$ with respect to a Cartesian reference frame with origin $\mathcal{O}$ and three mutually perpendicular base vectors $\left\{\boldsymbol{i}_{1}, \boldsymbol{i}_{2}, \boldsymbol{i}_{3}\right\}$ of unit length each. In the indicated order, the base vectors form a right-handed system. The time-domain electromagnetic field quantities are transformed with a Fourier transformation to the frequency domain according to

$$
\{\hat{\boldsymbol{E}}(\boldsymbol{x}, j \omega), \hat{\boldsymbol{H}}(\boldsymbol{x}, j \omega)\}=\int_{-\infty}^{\infty}\{\boldsymbol{E}(\boldsymbol{x}, t), \boldsymbol{H}(\boldsymbol{x}, t)\} \exp (-j \omega t) d t
$$

The electromagnetic properties are characterized by their permittivity $\epsilon$, permeability $\mu$ and the conductivity $\sigma$. In a steady-state analysis, all electromagnetic field quantities are taken to depend sinusoidally on time with a common angular frequency $\omega$. To each purely real space-time field quantity $\mathrm{f}(\boldsymbol{x}, \mathrm{t})$ we can associate a complex phasor $\hat{f}(\boldsymbol{x}, \omega)$ and a common time factor $e^{j \omega t}$, which are related through

$$
f(\boldsymbol{x}, t)=\operatorname{Re}\left\{\hat{f}(\boldsymbol{x}, j \omega) e^{j \omega t}\right\}
$$

Since in our analysis all the field quantities are in the frequency domain we will omit the ${ }^{\wedge}$ symbol from now on for convenience.

\subsection{Electromagnetic field equations and constitutive equations}

The electromagnetic field is governed by Maxwell's equations in the frequency domain:

$$
\begin{aligned}
-\nabla \times \boldsymbol{H}+\boldsymbol{J}+j \omega \boldsymbol{D} & =-\boldsymbol{J}^{e}, \\
\nabla \times \boldsymbol{E}+j \omega \boldsymbol{B} & =-\boldsymbol{K}^{e} .
\end{aligned}
$$


The names of the field quantities occurrring in eqs. (3.3) and (3.4) are

- $\boldsymbol{E}=$ electric field strength $\left(\mathrm{Vm}^{-1}\right)$

- $\boldsymbol{H}=$ magnetic field strength $\left(\mathrm{Am}^{-1}\right)$

- $\boldsymbol{D}=$ electric flux density $\left(\mathrm{Cm}^{-2}\right)$

- $\boldsymbol{B}=$ magnetic flux density $(\mathrm{T})$

- $\boldsymbol{J}=$ volume density of induced electric current $\left(\mathrm{Am}^{-2}\right)$

- $\boldsymbol{J}^{e}=$ volume source density of electric current $\left(\mathrm{Am}^{-2}\right)$

- $\boldsymbol{K}^{e}=$ volume source density of magnetic current $\left(\mathrm{Vm}^{-2}\right)$

The electromagnetic constitutive relations constitute the relationship between $\{\boldsymbol{D}, \boldsymbol{J}, \boldsymbol{B}\}$ and the electric and magnetic field strengths $\{\boldsymbol{E}, \boldsymbol{H}\}$. These relations are established by a physical experiment and are representative for the macroscopic electromagnetic properties of the materials present in the configuration. In our configuration we have linear, isotropic, locally reacting and time-invariant media, represented by the following constitutive relations

$$
\begin{aligned}
\boldsymbol{J}(\boldsymbol{x}, j \omega) & =\sigma(\boldsymbol{x}) \boldsymbol{E}(\boldsymbol{x}, j \omega), \\
\boldsymbol{D}(\boldsymbol{x}, j \omega) & =\epsilon(\boldsymbol{x}) \boldsymbol{E}(\boldsymbol{x}, j \omega), \\
\boldsymbol{B}(\boldsymbol{x}, j \omega) & =\overline{\boldsymbol{\mu}}(\boldsymbol{x}) \boldsymbol{H}(\boldsymbol{x}, j \omega),
\end{aligned}
$$

in which

$$
\begin{aligned}
\sigma(\boldsymbol{x}) & =\text { scalar conductivity }\left(\mathrm{Sm}^{-1}\right), \\
\epsilon(\boldsymbol{x}) & =\text { scalar permittivity }\left(\mathrm{Fm}^{-1}\right), \\
\overline{\boldsymbol{\mu}}(\boldsymbol{x}, j \omega) & =\text { tensorial permeability }\left(\mathrm{Hm}^{-1}\right) .
\end{aligned}
$$

For electromagnetic fields present in MRI we obtain according to Ibrahim [24], at the location of induced Magnetic Resonance due to the externally imposed RF field radiating at the Larmor frequency $\omega_{0}$ a tensorial permittivity. This results in the following relation for the magnetic flux density,

$$
\boldsymbol{B}\left(\boldsymbol{x}, j \omega_{0}\right)=\mu\left[\begin{array}{ccc}
1-j \gamma M_{0} T_{2} / 2 & -\gamma M_{0} T_{2} / 2 & 0 \\
\gamma M_{0} T_{2} / 2 & 1-j \gamma M_{0} T_{2} / 2 & 0 \\
0 & 0 & 1
\end{array}\right] \boldsymbol{H}\left(\boldsymbol{x}, j \omega_{0}\right),
$$

in which $M_{0}$ is the equilibrium static magnetization due to the static magnetic field $\boldsymbol{B}_{0}$ and $T_{2}$ is the spin spin relaxation time which relates to the decay of magnetization in the transverse plane after excitation with a RF pulse. The gyromagnetic ratio $\gamma$ of the region of interest relates the field strength $\boldsymbol{B}_{0}$ to the spin precession frequency $\omega_{0}$ (the Larmor frequency). Outside the region of induced Magnetic resonance when $\omega \neq \omega_{0}$ the tensorial permeability $\overline{\boldsymbol{\mu}}$ reduces to the scalar permeability $\mu$ as the matrix in Eq. (3.8) changes into the unit matrix, which yields

$$
\boldsymbol{B}(\boldsymbol{x}, j \omega)=\mu \boldsymbol{H}(\boldsymbol{x}, j \omega), \text { when } \omega \neq \omega_{0} .
$$

\subsection{The boundary conditions}

Across the interfaces where $\sigma, \epsilon$ and/or $\bar{\mu}$ show a jump discontinuity, the tangential components of the electric and magnetic field strengths are to be continuous. Let $\mathcal{S}$ denote the interface 


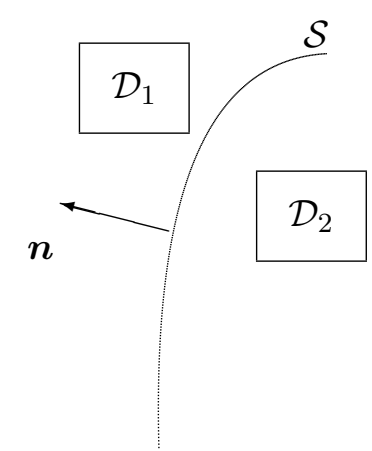

Figure 3.1: Interface between two media with different electromagnetic properties.

and assume that $\mathcal{S}$ has everywhere a unique tangent plane. Let, further, $\boldsymbol{n}$ denote the unit vector along the normal to $\mathcal{S}$ such that upon traversing $\mathcal{S}$ in the direction of $\boldsymbol{n}$, we pass from the domain $\mathcal{D}_{2}$ to the domain $\mathcal{D}_{1}, \mathcal{D}_{1}$ and $\mathcal{D}_{2}$ being located at either side of $\mathcal{S}$ (Fig. 3.1). Then at the boundary $\mathcal{S}$ we have the following boundary conditions

$$
\boldsymbol{n} \times \boldsymbol{E}(\boldsymbol{x}, j \omega) \text { is continuous across } \mathcal{S},
$$

and

$$
\boldsymbol{n} \times \boldsymbol{H}(\boldsymbol{x}, j \omega) \text { is continuous across } \mathcal{S} \text {. }
$$

\subsection{Time Fourier-transform domain field reciprocity theorem}

In this section the time Fourier-transform domain reciprocity theorem or Lorentz's reciprocity theorem [23] is discussed. A reciprocity relation interrelates in a specific manner the field quantities of two non-identical physical states that can occur in one and the same (bounded) domain in space. Let this domain be denoted by $\mathcal{D}$ with an enclosing surface $\partial \mathcal{D}$. The unit vector along the normal to $\partial \mathcal{D}$ is denoted by $\boldsymbol{n}$ and points away from $\mathcal{D}$. The complement of $\mathcal{D} \cup \partial \mathcal{D}$ in $\mathbb{R}^{3}$ is denoted by $\mathcal{D}^{\prime}$ (see Fig. 3.1). The two electromagnetic states that can occur in the domain $\mathcal{D}$ are referred to as states $A$ and $B$ (Table 1). Neither the media nor the sources present in the two states need be the same. State $A$ is characterized by the electromagnetic field

$$
\{\boldsymbol{E}, \boldsymbol{H}\}(\boldsymbol{x}, j \omega)=\left\{\boldsymbol{E}^{A}, \boldsymbol{H}^{A}\right\}(\boldsymbol{x}, j \omega),
$$

together with the constitutive parameters

$$
\{\sigma, \epsilon, \overline{\boldsymbol{\mu}}\}(\boldsymbol{x})=\left\{\sigma^{A}, \epsilon^{A}, \boldsymbol{\mu}^{A}\right\}(\boldsymbol{x}),
$$

and the volume source distributions

$$
\{\boldsymbol{J}, \boldsymbol{K}\}(\boldsymbol{x}, j \omega)=\left\{\boldsymbol{J}^{A}, \boldsymbol{K}^{A}\right\}(\boldsymbol{x}, j \omega) .
$$

The time Fourier-transform domain electromagnetic field of state $A$ satisfies the partial differential equations

$$
\begin{aligned}
-\nabla \times \boldsymbol{H}^{A}+\sigma^{A} \boldsymbol{E}^{A}+j \omega \epsilon^{A} \boldsymbol{E}^{A} & =-\boldsymbol{J}^{A}, \\
\nabla \times \boldsymbol{E}^{A}+j \omega \overline{\boldsymbol{\mu}}^{A} \boldsymbol{H}^{A} & =-\boldsymbol{K}^{A} .
\end{aligned}
$$


Table 3.1: The two electromagnetic states in the domain $\mathcal{D}$ in the time Laplace transform domain reciprocity theorem.

\begin{tabular}{|c|c|c|}
\hline \hline & State $A$ & State $B$ \\
\hline \hline Field state & $\left\{\boldsymbol{E}^{A}, \boldsymbol{H}^{A}\right\}(\boldsymbol{x}, j \omega)$ & $\left\{\boldsymbol{E}^{B}, \boldsymbol{H}^{B}\right\}(\boldsymbol{x}, j \omega)$ \\
Material state & $\left\{\sigma^{A}, \epsilon^{A}, \overline{\boldsymbol{\mu}}^{A}\right\}$ & $\left\{\sigma^{B}, \epsilon^{B}, \overline{\boldsymbol{\mu}}^{B}\right\}$ \\
Source state & $\left\{\boldsymbol{J}^{A}, \boldsymbol{K}^{A}\right\}(\boldsymbol{x}, j \omega)$ & $\left\{\boldsymbol{J}^{B}, \boldsymbol{K}^{B}\right\}(\boldsymbol{x}, j \omega)$ \\
\hline \hline
\end{tabular}

Similarly, state $B$ is characterized by the electromagnetic field

$$
\{\boldsymbol{E}, \boldsymbol{H}\}(\boldsymbol{x}, j \omega)=\left\{\boldsymbol{E}^{B}, \boldsymbol{H}^{B}\right\}(\boldsymbol{x}, j \omega),
$$

together with the constitutive parameters

$$
\{\sigma, \epsilon, \overline{\boldsymbol{\mu}}\}(\boldsymbol{x})=\left\{\sigma^{B}, \epsilon^{B}, \boldsymbol{\mu}^{B}\right\}(\boldsymbol{x}),
$$

and the volume source distributions

$$
\{\boldsymbol{J}, \boldsymbol{K}\}(\boldsymbol{x}, j \omega)=\left\{\boldsymbol{J}^{B}, \boldsymbol{K}^{B}\right\}(\boldsymbol{x}, j \omega) .
$$

The time Fourier-transform domain electromagnetic field of state B satisfies the partial differential equations

$$
\begin{aligned}
-\nabla \times \boldsymbol{H}^{B}+\sigma^{B} \boldsymbol{E}^{B}+j \omega \epsilon^{B} \boldsymbol{E}^{B} & =-\boldsymbol{J}^{B}, \\
\nabla \times \boldsymbol{E}^{B}+j \omega \overline{\boldsymbol{\mu}}^{B} \boldsymbol{H}^{B} & =-\boldsymbol{K}^{B} .
\end{aligned}
$$

The fundamental interaction quantity between the two states to be considered is the divergence of the vectorial quantity $\nabla\left(\boldsymbol{E}^{A} \times \boldsymbol{H}^{B}-\boldsymbol{E}^{B} \times \boldsymbol{H}^{A}\right)$. Which can be written as

$$
\begin{aligned}
\nabla \cdot\left(\boldsymbol{E}^{A} \times \boldsymbol{H}^{B}-\boldsymbol{E}^{B} \times \boldsymbol{H}^{A}\right)= & -\boldsymbol{H}^{B} \cdot\left(\nabla \times \boldsymbol{E}^{A}\right)+\boldsymbol{E}^{A} \cdot\left(\nabla \times \boldsymbol{H}^{B}\right) \\
& +\boldsymbol{H}^{A} \cdot\left(\nabla \times \boldsymbol{E}^{B}\right)-\boldsymbol{E}^{B} \cdot\left(\nabla \times \boldsymbol{H}^{A}\right) .
\end{aligned}
$$

The four terms on the right hand side of Eq. (3.22) can be rewritten using the differential equations (3.15), (3.16), (3.20) and (3.21) for the two different states. By collecting the results, we end up with the local form of the time Fourier-transform domain reciprocity theorem for the fields of State $A$ and State $B$ as

$$
\begin{aligned}
\nabla \cdot\left(\boldsymbol{E}^{A} \times\right. & \left.\boldsymbol{H}^{B}-\boldsymbol{E}^{B} \times \boldsymbol{H}^{A}\right)=j \omega\left(\boldsymbol{H}^{B} \cdot \overline{\boldsymbol{\mu}}^{A} \boldsymbol{H}^{A}-\boldsymbol{H}^{A} \cdot \overline{\boldsymbol{\mu}}^{B} \boldsymbol{H}^{B}\right) \\
& -j \omega\left(\epsilon^{A}-\epsilon^{B}\right) \boldsymbol{E}^{B} \cdot \boldsymbol{E}^{A}-\left(\sigma^{A}-\sigma^{B}\right) \boldsymbol{E}^{B} \cdot \boldsymbol{E}^{A} \\
& +\boldsymbol{E}^{A} \cdot \boldsymbol{J}^{B}-\boldsymbol{E}^{B} \cdot \boldsymbol{J}^{A}-\boldsymbol{H}^{A} \cdot \boldsymbol{K}^{B}+\boldsymbol{H}^{B} \cdot \boldsymbol{K}^{A} .
\end{aligned}
$$


Integration of Eq. (3.23) over the domain $\mathcal{D}$ with boundary $\partial \mathcal{D}$ and the use of Gauss' integral theorem in the resulting left-hand side leads to

$$
\begin{aligned}
& \iint_{\boldsymbol{x} \in \partial \mathcal{D}} \boldsymbol{n} \cdot\left(\boldsymbol{E}^{A} \times \boldsymbol{H}^{B}-\boldsymbol{E}^{B} \times \boldsymbol{H}^{A}\right) d A= \\
& \quad \iiint_{\boldsymbol{x} \in \mathcal{D}}\left[j \omega\left(\boldsymbol{H}^{B} \cdot \overline{\boldsymbol{\mu}}^{A} \boldsymbol{H}^{A}-\boldsymbol{H}^{A} \cdot \overline{\boldsymbol{\mu}}^{B} \boldsymbol{H}^{B}\right)-j \omega\left(\epsilon^{A}-\epsilon^{B}\right) \boldsymbol{E}^{B} \cdot \boldsymbol{E}^{A}\right. \\
& \left.\quad-\left(\sigma^{A}-\sigma^{B}\right) \boldsymbol{E}^{B} \cdot \boldsymbol{E}^{A}\right] d V+\iiint_{\boldsymbol{x} \in \mathcal{D}}\left[\boldsymbol{E}^{A} \cdot \boldsymbol{J}^{B}-\boldsymbol{E}^{B} \cdot \boldsymbol{J}^{A}\right. \\
& \left.\quad-\boldsymbol{H}^{A} \cdot \boldsymbol{K}^{B}+\boldsymbol{H}^{B} \cdot \boldsymbol{K}^{A}\right] d V,
\end{aligned}
$$

which is the global form of the time Fourier-transform domain reciprocity theorem. It is noted that the first three terms on the right-hand side of Eq. (3.23), as well as the first integral on the right-hand side of Eq. (3.24), vanish in case the media in the two states are chosen such that $\sigma^{A}=\sigma^{B}, \epsilon^{A}=\epsilon^{B}$ and $\overline{\boldsymbol{\mu}}^{A}=\left(\overline{\boldsymbol{\mu}}^{B}\right)^{T}$. If in addition the source distributions vanish in some domain, the corresponding local and global interactions are zero in that domain.

\subsection{The electromagnetic RF field in the excitation state}

In this section we consider the electromagnetic field with frequency $\omega_{0}$ that excites the magnetic contrast sources due to the tensorial permeability defined in Eq. (3.8). We will employ Lorentz's reciprocity theorem as defined in Eq (3.24). In order to obtain representations for the electromagnetic field inside the human body when the body is illuminated by an electromagnetic field operating at the Larmor freqeuncy $\omega_{0}$, we distinguish two different states in Lorentz's reciprocity theorem. The excitation state is the state where the generated electromagnetic fields $\left\{\boldsymbol{E}^{e}, \boldsymbol{H}^{e}\right\}$ in the entire domain $\mathbb{R}$ are excited by a current source distribution $\boldsymbol{J}^{e}$ inside a domain $\mathcal{S}$ (i.e. the domain of the exciting RF birdcage antenna) that illuminates the body that has a certain permittivity, conductivity and permeability distribution. While, the other state with electromagnetic fields $\left\{\boldsymbol{E}^{b}, \boldsymbol{H}^{b}\right\}$ is the field that is present in a homogeneous background medium and is generated by an electric current source or an magnetic current source. We can refer to this state as a Green's state. When we use an electric current point source in this Green's state, we will obtain in Lorentz's reciprocity theorem an integral equation representation for the electric field $\boldsymbol{E}^{e}$. On the other hand when we use a magnetic current point source in this Green's state, we will obtain in Lorentz's reciprocity theorem an integral equation representation for the magnetic field $\boldsymbol{H}^{e}$.

Since in the excitation state we want to know the magnetic field at the location of the induced magnetic resonance, we choose for the Green's state an excitation by a unit magnetic current point source located in a homogeneous medium with permittivity $\epsilon_{b}$, conductivity $\sigma_{b}$ and permeability $\mu_{b}$. For the two states in the excitation state we then have in Lorentz's reciprocity theorem (3.24),

$$
\begin{aligned}
\left\{\boldsymbol{E}^{A}, \boldsymbol{H}^{A}\right\} & =\left\{\boldsymbol{E}^{e}, \boldsymbol{H}^{e}\right\} \\
\left\{\boldsymbol{J}^{A}, \boldsymbol{K}^{A}\right\} & =\left\{\boldsymbol{J}^{e}, 0\right\}, \text { when } \boldsymbol{x} \in \mathcal{S} \\
\epsilon^{A} & =\epsilon^{e}(\boldsymbol{x}) \\
\sigma^{A} & =\sigma^{e}(\boldsymbol{x}) \\
\overline{\boldsymbol{\mu}}^{A} & =\overline{\boldsymbol{\mu}}^{e}\left(\boldsymbol{x}, j \omega_{0}\right),
\end{aligned}
$$


in which $\overline{\boldsymbol{\mu}}^{e}\left(\boldsymbol{x}, j \omega_{0}\right)$ is defined by Eq. (3.8), which reduces to a scalar permeability $\mu^{e}(\boldsymbol{x})$ when $\omega \neq \omega_{0}$.

The Green's state for a unit magnetic current point source $\boldsymbol{K}^{B}=\delta\left(\boldsymbol{x}-\boldsymbol{x}^{\prime}\right) \boldsymbol{u}$, where $\delta\left(\boldsymbol{x}-\boldsymbol{x}^{\prime}\right)$ represents the three-dimensional unit impulse (Dirac distribution) operative at $\boldsymbol{x}=\boldsymbol{x}^{\prime}$ and $\boldsymbol{u}$ represents the unit amplitude vector with $u_{1}=u_{2}=u_{3}=1$. The unit magnetic source is located inside the homogeneous domain with permittivity $\epsilon_{b}$, conductivity $\sigma_{b}$ and permeability $\mu_{b}$. Which yields for state $B$

$$
\begin{aligned}
\left\{\boldsymbol{E}^{B}, \boldsymbol{H}^{B}\right\} & =\left\{\boldsymbol{E}^{K ; b}, \boldsymbol{H}^{K ; b}\right\}\left(\boldsymbol{x}, \boldsymbol{x}^{\prime}, j \omega\right) \\
& =\left\{\overline{\boldsymbol{G}}^{E K}, \overline{\boldsymbol{G}}^{H K}\right\}\left(\boldsymbol{x}, \boldsymbol{x}^{\prime}, j \omega\right) \boldsymbol{u} \\
\left\{\boldsymbol{J}^{B}, \boldsymbol{K}^{B}\right\} & =\left\{0, \delta\left(\boldsymbol{x}-\boldsymbol{x}^{\prime}\right) \boldsymbol{u}\right\} \\
\epsilon^{B} & =\epsilon_{b} \\
\sigma^{B} & =\sigma_{b} \\
\overline{\boldsymbol{\mu}}^{B} & =\mu_{b} \overline{\boldsymbol{I}},
\end{aligned}
$$

where $\overline{\boldsymbol{G}}^{E K}$ represents the electric-field/magnetic-current Green's tensor, $\overline{\boldsymbol{G}}^{H K}$ represents the magnetic-field/magnetic-current Green's tensor. Similarly, the Green's tensor $\bar{G}^{E J}$ represents the electric-field/electric-current Green's tensor and $\bar{G}^{H J}$ represents the magnetic-field/electriccurrent Green's tensor.

Now for a homogeneous, isotropic medium with permitttivity $\epsilon_{b}$, conductivity $\sigma_{b}$ and permeability $\mu_{b}$ we obtain for the Green's tensors [11] due to a unit excitation,

$$
\begin{aligned}
\overline{\boldsymbol{G}}^{E J} \boldsymbol{u} & =-j \omega \mu_{b} G\left(\boldsymbol{x}, \boldsymbol{x}^{\prime}\right) \overline{\boldsymbol{I}} \boldsymbol{u}+\left(j \omega \epsilon_{b}+\sigma_{b}\right)^{-1} \nabla\left(\nabla \cdot G\left(\boldsymbol{x}, \boldsymbol{x}^{\prime}\right) \boldsymbol{u}\right), \\
\overline{\boldsymbol{G}}^{E K} \boldsymbol{u} & =-\nabla \times G\left(\boldsymbol{x}, \boldsymbol{x}^{\prime}\right) \boldsymbol{u}, \\
\overline{\boldsymbol{G}}^{H J} \boldsymbol{u} & =\nabla \times G\left(\boldsymbol{x}, \boldsymbol{x}^{\prime}\right) \boldsymbol{u}, \\
\overline{\boldsymbol{G}}^{H K} \boldsymbol{u} & =-\left(j \omega \epsilon_{b}+\sigma_{b}\right) G\left(\boldsymbol{x}, \boldsymbol{x}^{\prime}\right) \overline{\boldsymbol{I}} \boldsymbol{u}+\left(j \omega \mu_{b}\right)^{-1} \nabla\left(\nabla \cdot G\left(\boldsymbol{x}, \boldsymbol{x}^{\prime}\right) \boldsymbol{u}\right),
\end{aligned}
$$

in which

$$
G\left(\boldsymbol{x}, \boldsymbol{x}^{\prime}\right)=\frac{\exp \left(-j k\left|\boldsymbol{x}-\boldsymbol{x}^{\prime}\right|\right.}{4 \pi\left|\boldsymbol{x}-\boldsymbol{x}^{\prime}\right|}, \text { for } \boldsymbol{x} \neq \boldsymbol{x}^{\prime} \text { and } k=\omega\left[\epsilon_{b} \mu_{b}+\sigma_{b} /(j \omega)\right]^{1 / 2}
$$

The above Eqs. (3.35) and (3.37) are both solutions to Maxwell's equations Eq. 3.3 and Eq. 3.4 for an electric current point source $\boldsymbol{u}$ for $\boldsymbol{J}=\delta\left(\boldsymbol{x}-\boldsymbol{x}^{\prime}\right)$ and $\boldsymbol{K}=\mathbf{0}$,

$$
\begin{aligned}
\boldsymbol{E}\left(\boldsymbol{x}, \boldsymbol{x}^{\prime}, j w\right) & =\overline{\boldsymbol{G}}^{E J}\left(\boldsymbol{x}, \boldsymbol{x}^{\prime}, j w\right) \boldsymbol{u} \\
\boldsymbol{H}\left(\boldsymbol{x}, \boldsymbol{x}^{\prime}, j w\right) & =\overline{\boldsymbol{G}}^{H J}\left(\boldsymbol{x}, \boldsymbol{x}^{\prime}, j w\right) \boldsymbol{u}
\end{aligned}
$$

Equally, the above Eqs. (3.36) and (3.38) are both solutions to Maxwell's equations Eq. (3.3) and (3.4) for a magnetic current point source $\boldsymbol{u}$ for $\boldsymbol{K}=\delta\left(\boldsymbol{x}-\boldsymbol{x}^{\prime}\right)$ and $\boldsymbol{J}=\mathbf{0}$,

$$
\begin{aligned}
\boldsymbol{E}\left(\boldsymbol{x}, \boldsymbol{x}^{\prime}, j w\right) & =\overline{\boldsymbol{G}}^{E K}\left(\boldsymbol{x}, \boldsymbol{x}^{\prime}, j w\right) \boldsymbol{u} \\
\boldsymbol{H}\left(\boldsymbol{x}, \boldsymbol{x}^{\prime}, j w\right) & =\overline{\boldsymbol{G}}^{H K}\left(\boldsymbol{x}, \boldsymbol{x}^{\prime}, j w\right) \boldsymbol{u}
\end{aligned}
$$


Finally, $\overline{\boldsymbol{I}}$ represents the unit tensor and all tensors are of rank 3.

Application of Lorentz's reciprocity theorem cf. $\mathrm{Eq}(3.24)$ in which we let $\partial \mathcal{D}$ extend to infinity (unbounded domain) yields,

$$
\begin{aligned}
\iiint_{\boldsymbol{x} \in \mathcal{D}}\left[j \omega \left(\boldsymbol{H}^{B} \cdot \overline{\boldsymbol{\mu}}^{A} \boldsymbol{H}^{A}\right.\right. & \left.-\boldsymbol{H}^{A} \cdot \overline{\boldsymbol{\mu}}^{B} \boldsymbol{H}^{B}\right)-j \omega\left(\epsilon^{A}-\epsilon^{B}\right) \boldsymbol{E}^{B} \cdot \boldsymbol{E}^{A} \\
& -\left(\sigma^{A}-\sigma^{B}\right) \boldsymbol{E}^{B} \cdot \boldsymbol{E}^{A}+\boldsymbol{E}^{A} \cdot \boldsymbol{J}^{B}-\boldsymbol{E}^{B} \cdot \boldsymbol{J}^{A} \\
& \left.-\boldsymbol{H}^{A} \cdot \boldsymbol{K}^{B}+\boldsymbol{H}^{B} \cdot \boldsymbol{K}^{A}\right] d V=0
\end{aligned}
$$

since according to the radiation condition of the electromagnetic fields at infinity, the contribution of the surface integral vanishes.

Substitution of the fields and sources of the corresponding states in Eq. (3.45), using the fact that for a general field/source vector $\boldsymbol{F}$

$$
\overline{\boldsymbol{G}}^{E K}\left(\boldsymbol{x}, \boldsymbol{x}^{\prime}, j \omega\right) \boldsymbol{u} \cdot \boldsymbol{F}=\boldsymbol{u} \cdot\left(\overline{\boldsymbol{G}}^{E K}\right)^{T}\left(\boldsymbol{x}^{\prime}, \boldsymbol{x}, j \omega\right) \boldsymbol{F}=-\boldsymbol{u} \cdot\left(\overline{\boldsymbol{G}}^{H J}\right)^{T}\left(\boldsymbol{x}^{\prime}, \boldsymbol{x}, j \omega\right) \boldsymbol{F},
$$

and

holds, we obtain

$$
\overline{\boldsymbol{G}}^{H K}\left(\boldsymbol{x}, \boldsymbol{x}^{\prime}, j \omega\right) \boldsymbol{u} \cdot \boldsymbol{F}=\boldsymbol{u} \cdot\left(\overline{\boldsymbol{G}}^{H K}\right)^{T}\left(\boldsymbol{x}^{\prime}, \boldsymbol{x}, j \omega\right) \boldsymbol{F},
$$

$$
\begin{aligned}
\boldsymbol{H}^{e}\left(\boldsymbol{x}^{\prime}\right)= & \iiint_{\boldsymbol{x} \in \mathcal{V}}\left[j \omega\left(\overline{\boldsymbol{G}}^{H K}\right)^{T}\left(\boldsymbol{x}^{\prime}, \boldsymbol{x}, j \omega\right)\left(\overline{\boldsymbol{\mu}}^{e}(\boldsymbol{x})-\mu_{b} \overline{\boldsymbol{I}}\right) \boldsymbol{H}^{e}(\boldsymbol{x})\right. \\
& +\left[j \omega\left(\epsilon^{e}(\boldsymbol{x})-\epsilon_{b}\right)+\left(\sigma^{e}(\boldsymbol{x})-\sigma_{b}\right)\right]\left(\overline{\boldsymbol{G}}^{H J}\right)^{T}\left(\boldsymbol{x}^{\prime}, \boldsymbol{x}, j \omega\right) \boldsymbol{E}^{e}(\boldsymbol{x}) d V \\
& +\iiint_{\boldsymbol{x} \in \mathcal{S}}\left(\overline{\boldsymbol{G}}^{H J}\right)^{T}\left(\boldsymbol{x}^{\prime}, \boldsymbol{x}, j \omega\right) \boldsymbol{J}^{e}(\boldsymbol{x}) d V, \text { for } \boldsymbol{x}^{\prime} \in \mathbb{R}^{3}
\end{aligned}
$$

which is the magnetic field in the excitation state in which the domain $\mathcal{V}$ is the domain that contains the illuminated human body and the domain $\mathcal{S}$ is the domain containing the illuminating $\mathrm{RF}$ antenna. The first term of Eq. 3.48 describes the contrast in $\mu$, which only exists in the imaging slice during excitation. The magnetic contrast is compared to the electric contrasts in the body negligibly small. Induced electric fields at the electric contrasts described in the second term do attribute to the magnetic field. Tissue with a high conductivity will absorb part of the electric field, turning it into heat. There is not so much we can do about this, a generated magnetic field will always have an electric field present. However for the retainer wire, we can store the electric fields around the wire, such that the energy can not be absorbed directly around the wire. Electric field storage is possible for a spacer with zero conductivity following the complex poynting theorem 2.18.

The calculated magnetic field $\boldsymbol{H}^{e}\left(\boldsymbol{x}^{\prime}\right)$ in the integral equation, Eq. (3.48) is related to the the electric field through Maxwell's equations Eq. (3.16)

$$
\boldsymbol{H}^{e}\left(\boldsymbol{x}^{\prime}\right)=-\frac{1}{j \omega \mu_{0}} \nabla \times \boldsymbol{E}^{e}\left(\boldsymbol{x}^{\prime}\right)
$$

A change in the magnetic field has a a change in the electric field as well, which couples through the second term of equation Eq. (3.48) back to the magnetic field.

This coupling follows from the second maxwell's equation, Eq. (3.16) for state A, with $\boldsymbol{x}^{\prime}$ in the source-free domain $\left(\boldsymbol{x}^{\prime} \notin \mathcal{S}\right)$ in combination with fields described in Eq. (3.25) and sources described in Eq. (3.26). The antenna is described in the third term of Eq. (3.48) by an electric current source $\boldsymbol{J}^{e}(\boldsymbol{x})$. The magnetic field $\boldsymbol{H}^{e}\left(\boldsymbol{x}^{\prime}\right)$ in Eq. (3.48) is excited by the current $\boldsymbol{J}^{e}(\boldsymbol{x})$. 


\subsection{The electromagnetic $\mathrm{RF}$ field in the receiving state}

In this section we consider the electromagnetic field with frequency $\omega_{0}$ that is received by receiving antennas and is excited by the magnetic contrast sources due to the tensorial permeability defined in Eq. (3.8). We will employ Lorentz's reciprocity theorem as defined in Eq (3.24). In order to obtain representations for the electromagnetic field at the receiving antennas. Again we distinguish two different states in Lorentz's reciprocity theorem. The receiving state is the state where the emitted electromagnetic fields $\left\{\boldsymbol{E}^{e}, \boldsymbol{H}^{e}\right\}$ in the entire domain $\mathbb{R}$ are excited by the magnetic contrast source distribution $\boldsymbol{K}^{r}$ inside a domain $\mathcal{K}$ (i.e. the domain that contains the slice of excited magnetic contrast sources or magnetic moments with larmor frequency $\omega_{0}$ ) obtained from the excitation state in the previous section,

$$
\boldsymbol{K}^{r}(\boldsymbol{x})=j \omega\left(\overline{\boldsymbol{\mu}}^{e}(\boldsymbol{x})-\mu_{b} \overline{\boldsymbol{I}}\right) \boldsymbol{H}^{e}(\boldsymbol{x}), \text { with } \boldsymbol{x} \in \mathcal{K} \text {. }
$$

While, the other state with electromagnetic fields $\left\{\boldsymbol{E}^{b}, \boldsymbol{H}^{b}\right\}$ is the field that is present in a homogeneous background medium and is generated by an electric current source or an magnetic current source. We can refer to this state as a Green's state.

Since in the receiving state we want to know the magnetic field at the location of the receiving antennas $\boldsymbol{H}^{\boldsymbol{r}}\left(\boldsymbol{x}^{\prime}\right)$, we choose for the Green's state an excitation by an unit magnetic current point source located in a homogeneous medium with permittivity $\epsilon_{b}$, conductivity $\sigma_{b}$ and permeability $\mu_{b}$. For the two states in the receiving state we then have in Lorentz's reciprocity theorem $(3.24)$,

$$
\begin{aligned}
\left\{\boldsymbol{E}^{A}, \boldsymbol{H}^{A}\right\} & =\left\{\boldsymbol{E}^{r}, \boldsymbol{H}^{r}\right\} \\
\left\{\boldsymbol{J}^{A}, \boldsymbol{K}^{A}\right\} & =\left\{0, \boldsymbol{K}^{r}\right\}, \text { when } \boldsymbol{x} \in \mathcal{K} \\
\epsilon^{A} & =\epsilon^{r}(\boldsymbol{x}) \\
\sigma^{A} & =\sigma^{r}(\boldsymbol{x}) \\
\overline{\boldsymbol{\mu}}^{A} & =\mu_{b} \overline{\boldsymbol{I}}
\end{aligned}
$$

The Green's state for a unit magnetic current point source $\boldsymbol{K}^{B}=\delta\left(\boldsymbol{x}-\boldsymbol{x}^{\prime}\right) \boldsymbol{u}$, where $\delta\left(\boldsymbol{x}-\boldsymbol{x}^{\prime}\right)$ represents the three-dimensional unit impulse (Dirac distribution) operative at $\boldsymbol{x}=\boldsymbol{x}^{\prime}$ and $\boldsymbol{u}$ represents the unit amplitude vector with $u_{1}=u_{2}=u_{3}=1$. The unit magnetic source is located inside the homogeneous domain with permittivity $\epsilon_{b}$, conductivity $\sigma_{b}$ and permeability $\mu_{b}$, which yields for state $B$

$$
\begin{aligned}
\left\{\boldsymbol{E}^{B}, \boldsymbol{H}^{B}\right\} & =\left\{\boldsymbol{E}^{K ; b}, \boldsymbol{H}^{K ; b}\right\}\left(\boldsymbol{x}, \boldsymbol{x}^{\prime}, j \omega\right) \\
& =\left\{\overline{\boldsymbol{G}}^{E K}, \overline{\boldsymbol{G}}^{H K}\right\}\left(\boldsymbol{x}, \boldsymbol{x}^{\prime}, j \omega\right) \boldsymbol{u} \\
\left\{\boldsymbol{J}^{B}, \boldsymbol{K}^{B}\right\} & =\left\{0, \delta\left(\boldsymbol{x}-\boldsymbol{x}^{\prime}\right) \boldsymbol{u}\right\} \\
\epsilon^{B} & =\epsilon_{b} \\
\sigma^{B} & =\sigma_{b} \\
\overline{\boldsymbol{\mu}}^{B} & =\mu_{b} \overline{\boldsymbol{I}} .
\end{aligned}
$$

Application of Lorentz reciprocity theorem Eq. (3.45) in combination with the definitions for the fields in state A and B in receiving mode Eqs. (3.51) to (3.60) yields the formulation of the 
received magnetic field

$$
\begin{aligned}
\boldsymbol{H}^{r}\left(\boldsymbol{x}^{\prime}\right)= & \iiint_{\boldsymbol{x} \in \mathcal{V}}\left[j \omega\left(\epsilon^{r}(\boldsymbol{x})-\epsilon_{b}\right)+\sigma^{r}(\boldsymbol{x})-\sigma_{b}\right]\left(\overline{\boldsymbol{G}}^{H J}\right)^{T}\left(\boldsymbol{x}, \boldsymbol{x}^{\prime}, j \omega\right) \boldsymbol{E}^{r}(\boldsymbol{x}) d V \\
& +\iiint_{\boldsymbol{x} \in \mathcal{K}}\left(\overline{\boldsymbol{G}}^{H K}\right)^{T}\left(\boldsymbol{x}, \boldsymbol{x}^{\prime}, j \omega\right) \boldsymbol{K}^{r}(\boldsymbol{x}) d V, \text { for } \boldsymbol{x}^{\prime} \in \mathbb{R}^{3}
\end{aligned}
$$

In which $\mathcal{V}$ is the volume containing the body and receiving antenna's. $\mathcal{K}$ is the source domain, containing the volume of excited magnetic moments that are illuminableminated in the excitation state on the Larmor frequency. These excited magnetic moments are represented by the magnetic current source $\boldsymbol{K}^{r}(\boldsymbol{x})$. The first term indicates that the induced electric currents at the electric contrasts also attribute to the received magnetic field at the receiver. The contribution of the $\boldsymbol{K}^{r}$ source to the received magnetic field $\boldsymbol{H}^{r}\left(\boldsymbol{x}^{\prime}\right)$ is described in the second term of Eq. (3.61).

The received magnetic field $\boldsymbol{H}^{r}\left(\boldsymbol{x}^{\prime}\right)$ is related to the electric field as described by Maxwell's second equation, Eq. 3.16, with fields, sources and media for state A defined in Eqs. (3.51) and (3.52). For the region outside the slice containing the magnetic sources $\left(\boldsymbol{x}^{\prime} \notin \mathcal{K}\right)$

$$
\boldsymbol{H}^{r}\left(\boldsymbol{x}^{\prime}\right)=-\frac{1}{j \omega \mu_{0}} \nabla \times \boldsymbol{E}^{r}\left(\boldsymbol{x}^{\prime}\right)
$$

A change in the magnetic field has a a change in the electric field as well, which couples through the second term of equation Eq. (3.61) back to the magnetic field. 


\section{Chapter 4}

\section{Tissue heating around implants}

\subsection{Cause of possible heating around a metal implant}

This research focuses on the electromagnetic problem of a field with known field strength impinging on a wire shaped metallic object within a dielectric body. All dimensions, positions and electromagnetic properties of the object and medium are known, so this is forward problem. The incident field created by an RF coil will induce a conducting current along the wire and in the tissue which has two effects leading to tissue heating.

The first effect is due the lossy metal wire which will dissipate some of the electric current and heat not only itself but also transfer its heat to the directly surrounding tissue depending on thermal conductivity. Secondly, the current in the wire will flow into the surrounding tissue focussing at the wire tips. This conduction current, together with the already induced tissue currents, will (partly) be dissipated with a rise in temperature as a consequence. The amount of energy being dissipated and how this is leading to a temperature rise is described in the following section.

\subsubsection{Temperature rise due to dissipated energy}

Tissue dissipated power leads to a temperature rise which can be described by Penne's bio heat equation. This equation has the dissipated power and metabolic heat as a source for heating, while the body is cooled through perfusion, heat conduction and convection. The rate of temperature change $(\mathrm{d} T / \mathrm{d} t)$ is tissue specific, governed by its density $(\rho)$ and heat capacity (c) according to

$$
c \rho \frac{\mathrm{d} T}{\mathrm{~d} t}=P_{\text {diss }}-P_{\text {cooling }}+P_{\text {metabolic }}
$$

This is Penne's bioheat equation, in which $c$ is the heat capacity of tissue in $\frac{J}{k g}{ }^{\circ} K, \rho$ the tissue specific density in $\frac{\mathrm{kg}}{\mathrm{m}^{3}}$ and $\frac{\mathrm{d} T}{\mathrm{~d} t}$ the temperature change per second. The body is actively cooling the tissue through blood perfusion and passively through heat conduction and air convection while the metabolic process generates heat. Simulations on the temperature rise around the retainer wire described in Section 5.3.2 by the electromagnetic and thermal solver CST are based on the bioheat equation (4.1). 


\subsubsection{Electromagnetic resonators}

The increased frequency used by the latest MR systems up to Ultra High Field MRI pushes the wavelength down as seen in Table 2.1. A large range of implants at $7 \mathrm{~T}$ can be categorized as resonating structure. As a rule of thumb, the resonating length is between $\lambda / 3$ and $\lambda / 2$, where $\lambda$ is the wavelength in the surrounding medium. The wavelength in a medium is defined as the distance traveled by a time harmonic planar electromagnetic wave, in one period $(2 \pi)$ of frequency $f(f=\omega / 2 \pi)$ of the wave. The speed and amplitude of a traveling planar wave can be described by a complex propagation constant $\gamma_{\text {prop }}$, [12]. The following relation describes the electric field for a planar wave propagating away over the z-direction.

$$
\begin{aligned}
\boldsymbol{E}(z) & =\boldsymbol{E}_{0} e^{-\gamma_{\text {propagation }} z} \\
& =\boldsymbol{E}_{0} e^{-(\alpha+j \beta) z}
\end{aligned}
$$

With $\boldsymbol{E}_{0}$, the initial value of the electric field at $\mathrm{z}=0$ and $\gamma_{\text {prop }}$ given by,

$$
\begin{aligned}
& \gamma_{\text {prop }}=\sqrt{j \omega \mu(\sigma+j \omega \epsilon)} \\
& \gamma_{\text {prop }}=\alpha+j \beta
\end{aligned}
$$

The imaginary part of $\gamma_{\text {prop }}$ represents the phase speed, $\beta$, of the wave in radials per meter. The real part of $\gamma_{\text {prop }}$ represents the attenuation of the wave while traveling over $\mathrm{z}$. The phase constant $\beta$ is calculated from the imaginary part of $\sqrt{j \omega \mu(\sigma+j \omega \epsilon)}$ which is equal to

$$
\beta=\omega \sqrt{\mu \epsilon}\left\{\frac{1}{2}\left[\sqrt{1+\left(\frac{\sigma}{\omega \epsilon}\right)^{2}}+1\right]\right\}^{\frac{1}{2}} \quad[\mathrm{rad} / \mathrm{m}]
$$

The wavelength $\lambda$ in meters is defined as the distance that a wave has traveled for one radial, equalling $2 \pi / \beta$. By using the fact that the speed of light in vacuum $c_{0}$ is equal to $\sqrt{\epsilon_{0} \mu_{0}}$ and $\omega=2 \pi f$, we write the wavelength $\lambda$ as

$$
\lambda=\frac{c_{0}}{\sqrt{\epsilon_{r} \mu_{r}} f}\left\{\frac{1}{2}\left[\sqrt{1+\left(\frac{\sigma}{2 \pi f \epsilon}\right)^{2}}+1\right]\right\}^{-\frac{1}{2}}[\mathrm{~m}]
$$

The resonant length for a wire, which as a rule of thumb is the half wavelength, is given by half the wavelength. The governing parameters of the wavelength are $f$ the RF frequency of the EM field which at 7 tesla is $298.2 \mathrm{MHz}$ together with the relative permittivity $\left(\epsilon_{r}\right)$, permeability $\left(\mu_{r}\right)$ and conductivity of the medium. For example, the half wavelength in muscle, which has a relative permittivity of 58.1, relative permeability of 1 and conductivity $0.7 \mathrm{~S} / \mathrm{m}$ at $298 \mathrm{MHz}$ (Table 4.2 ) is equal to $6.1 \mathrm{~cm}$.

Calculating the half wavelength in an heterogeneous medium requires a more sophisticated model. A retainer wire is located in an heterogeneous medium comprising of teeth, muscle, skin, air, saliva and dental glue. The current through the wire and its related fields interacts to a certain extend with each of these materials, making an analytical calculation of the wavelength very cumbersome. Attempts have been made to model implanted wires as a single wire transmission line, as described in the literature (following section). Most of these models still assume a homogeneous medium, making this form of wavelength prediction not suitable for the retainer wire. 
In this study we model the retainer wire in a homogeneous medium through a numerical EM solver to check whether the resonant length is in accordance to the rule of thumb. For each of the previously described surrounding materials (air, teeth, skin, muscle, saliva) the resonant length is calculated

Table 4.1: Wavelengths of materials surrounding the retainer wire at $298.2 \mathrm{MHz}$

\begin{tabular}{l|lll} 
& Conductivity $[\mathrm{S} / \mathrm{m}]$ & Relative permittivity & Half wavelength $[\mathrm{mm}]$ \\
\hline Air & 0 & 1 & 501 \\
Teeth & 0.083 & 14 & 132 \\
Skin (wet) & 0.63 & 51.9 & 65.8 \\
Tongue (muscle) & 0.7 & 58.2 & 61.6 \\
Saliva & 0.34 & 80 & 55.6
\end{tabular}

The half wavelengths are presented in Table 4.1 for a homogeneous medium only. However, in practice the wavelength will be a combination of lengths, based on a proximity weighted function. Since the teeth and the tongue will be closest to the wire, most likely the effective wavelength will be in the order of 61 to $132 \mathrm{~mm}$. The worst case scenario in terms of resonance length is found for Saliva, which has the shortest wavelength at $55.6 \mathrm{~mm}$. The longest length of retainer wire is $55 \mathrm{~mm}$, so in theory a fully resonant wire is possible. The next step is to determine to what extend the resonating effect boosts the SAR around the wire. We investigate this issue by carrying out simulations and measurements on wires of different lengths. In case the SAR values are (too) high we investigate how to reduce the absorbed power around in Section 4.2.1.

\subsubsection{Previous studies on wire heating}

Practical heating studies for MRI of dental retainer wires have not been reported at this point. Previous research on wire heating has been performed specifically for interventional MRI [5], [6], [7] and patients with implanted devices [17], [20], [30]. The majority of practical studies are focussed on $1.5 \mathrm{~T}$ and $3 \mathrm{~T}$.

A study on wires partly being submerged in a dielectric material and partly being in air as is happening for guide wires for interventional MRI [5] showed an increase of $48{ }^{\circ} \mathrm{C}$ at the wire. The measurement were performed in a $1.5 \mathrm{~T}$ scanner, on a straight $150 \mathrm{~cm}$ guide wire in $\mathrm{z}$ direction. Part of the line was submerged in a saline solution, while a second part of the line was suspended in air to simulate an endovascular intervention. A measured temperature increase was registered as large as $48{ }^{\circ} \mathrm{C}$ after 30 seconds of scanning. Touching the tip of the wire with a finger induced a high temperature in the skin.

A safety index that characterizes the increase in SAR in the tissue around a wire has been proposed by Yeung et al [7]. This theoretical and practical study reported on the influence of a change in the wire dimensions - diameter, length, insulation thickness, insulation permittivity and tissue conductivity to the SAR at $1.5 \mathrm{~T}$. The parameters of largest influence on SAR reduction found in the study are wire insulation thickness and tissue conductivity. The suggestion in the paper to design safer wires by applying insulation around the wire is further investigated in Section 4.2.1. An increase in tissue conductivity showed a decrease in SAR .

The results of the paper by Yeung provide a good prediction of what a change in the wire parameters will have as an effect, however the surrounding tissue in that study is limited to 
homogeneous material. Predicting whether a wire will be resonating is more difficult in a heterogenous volume. The body and especially the volume around the retainer wire are highly heterogeneous. A recent study has described a single wire as a modified transmission line in an attempt to predict resonance effects in a heterogeneous environment [14].

\subsection{SAR monitoring}

There are a variety of ways to monitor or predict the SAR in a patient. As described before, attempts have been made to the SAR around wires by analytical methods. A second possibility is that if a voxel model of the patient is available then electromagnetic simulations of the human model with a transmit coil can give an indication for the location of SAR hotspots and their strength. An other way is based on MR methods which have been developed to deduce the electric field from $\mathrm{MR} \mathrm{B}_{1}^{+}$measurements, although it has also been shown that for similar $\mathrm{B}_{1}^{+}$fields in the body different SAR distributions are found. This is due to the heterogeneous nature of the tissue, for which every tissue boundary introduces a contrast source.

An upcoming technique in MRI is electrical properties tomography (EPT) [18] in which an MR system is used to map the electrical properties of the patient into a voxel model. The model can thereafter be used for patient specific SAR prediction in an EM simulation. When this technique is fully mature, this could set out to be a good predictor of personalized local SAR and temperature distributions. A temperature measurement based technique is the SAR calorimetry method, in which the SAR is calculated from a temperature rise during a SAR intensive MR sequence. This method is relatively easy to setup using only a few temperature probes and is used in this study on a phantom model with a retainer wire.

\subsubsection{SAR calorimetry}

SAR around an implant can be calculated from the temperature rise measured by temperature probes. These two quantities are related through Penne's bioheat equation. A simplified version of Penne's full bioheat equation (Eq. (4.1)) relates temperature change linearly to the SAR . This simplified version assumes no thermal transport due to thermal conduction, perfusion and convection and is given by

$$
\frac{\mathrm{d} T}{\mathrm{~d} t}=\frac{P_{\text {diss_volume }}}{c \rho}=\frac{\mathrm{SAR}}{c}\left[{ }^{0} \mathrm{C} / \mathrm{s}\right],
$$

With,

$$
\mathrm{SAR}=c \frac{\mathrm{d} T}{\mathrm{~d} t} \quad[\mathrm{~W} / \mathrm{kg}] .
$$

An approximation for linearity in the bioheat equation is valid for in vivo tissue in the first few seconds of an EM heating experiment. The cooling mechanisms - convection, heat conduction to colder tissue and perfusion with colder fluids- start to play a role only after the temperature difference between heated tissue and surrounding tissue is significant. The use of the linear approximation underestimates the calculated SAR when employed beyond its linear region so only the first few seconds of a measurement deliver valid SAR values.

The heating during such a temperature measurement should be high enough to be measurable by lab temperature sensors and for human in-vivo testing, staying below the IEC limit of $38^{\circ} \mathrm{C}$ 
for the head. These contradicting terms make in-vivo SAR measurements impractical. However, the larger temperature limits of a phantom for heating experiments can be appreciated. The SAR distribution around a retainer wire in tissue will in this study be investigated using the SAR calorimetry method. Temperature measurements on a phantom can be a source of absolute SAR values, which can be linked to transmitted power for the EM simulations and to the temperature simulations of a phantom.

\subsection{Spacer around the retainer wire for SAR reduction}

In the literature on interventional MRI safety, electrically insulated [7] wires have been experimentally investigated. Wires with electrical insulation over the entire length are reported to have a lower SAR at the insulated tips compared to bare wires. This idea can be applied to the retainer wire if its presence is causing a breach in safety regulations for MRI. Full insulation around the retainer wire is practically not feasible unfortunately. Instead, the sensitive tissue (read: tongue) can be refrained from touching the wire which as described in literature is cause of potential local heating [5]. As is motivated in the next section, the spacer material has to be a low conductivity material and biologically safe such as dental wax or a silicone sports-mouthguard. Electrical properties of potential spacer materials are listed in Table 4.2.

Table 4.2: Materials surrounding a retainer wire, including potential SAR lowering spacer materials, marked *

\begin{tabular}{l|ll} 
& Conductivity $\mathrm{S} / \mathrm{m}$ & Relative permittivity \\
\hline Air & 0 & 1 \\
${ }^{*}$ Silicone rubber & $10^{-9}$ & 3 to 9 \\
${ }^{*}$ Bee Wax & $10^{-13}$ & 2 \\
${ }^{*}$ Paraffin wax & $10^{-13}$ & 7 \\
Teeth & 0.083 & 14 \\
Tongue (muscle) & 0.7 & 58.2 \\
Saliva & 0.34 & 80
\end{tabular}

The listed spacer materials [29] need to be of low conductivity such that induced currents cannot flow from the tissue into the wire and vise versa. A wire with a single bare tip is possibly more dangerous than no separation at all, as described in [19]. The use of dental wax, which could come off during a scan is therefore not advisable. The mouthguard together with the air surrounding the wire can form a fully enclosing low conductivity isolator.

\subsection{SAR reduction around the wire}

In EM simulations and measurements of wires embedded in an homogeneous medium it has been shown previously in literature that the SAR is largest around the tips [6],[7]. This means that most of the electric field energy around the wire is concentrated around the tips in the conductive tissue. In the following paragraphs, the cause for SAR localization at the tips is discussed together with reasoning on how SAR is reduced when a non conductive spacer is applied. 
In our experiment we consider: a metal wire, with a conductivity of $5 \cdot 10^{5} \mathrm{~S} / \mathrm{m}$ and a diameter $d$ $\ll \lambda$ and length $l \approx \lambda / 2$. In scenario 1 , a bare wire without any isolation is considered while in scenario 2 , the wire is being surrounded by a thin layer of non-conducting material and a low relative permittivity between 1 and 9 such as air, wax or silicone. The wires in both scenarios are submerged inside an homogeneous medium. This medium is taken as a phantom filled with a gel based on Hydroxy Ethyl Cellulose (HEC), water and Natrium Chloride as is used in the ASTM RF safety standard [21]. The medium properties are the following: the phantom gel has a relative permittivity and conductivity are 81 and $0.47 \mathrm{~S} / \mathrm{m}$ at $298.2 \mathrm{MHz}$. The source is taken as a wave traveling over the xy plane in direction of the wire which itself is pointing in the $\mathrm{x}$-direction. We let the origin of the source be rotating with the same frequency $298 \mathrm{MHz}$ over the xy-plane around the wire, like the fields created by a birdcage. The wire and its surrounding spacer can best be described in cylindrical coordinates $\left(\boldsymbol{i}_{r}, \boldsymbol{i}_{\theta}, \boldsymbol{i}_{z}^{\prime}\right)$ in which the wire is pointing in z' direction.

\subsubsection{Bare wire in tissue}

In the first scenario as described above an antenna is transmitting EM waves towards a bare wire in gel. The incident electric fields will induce a conduction current $\boldsymbol{J}^{c}$ in both wire and gel

$$
\boldsymbol{J}_{\text {wire }}^{c}=\sigma_{\text {wire }} \boldsymbol{E}_{\text {wire }}
$$

The electric fields reaching the highly conductive wire will vanish and generate a conduction current on the surface of the wire. Currents induced in the gel around the wire will add to the current over the wire. Both contributions flow along the direction of the wire in which the wire channels the currents of the surrounding tissue towards the wire tips. Since the tissue has a non-zero conductivity, part of the channeled current Eq. (4.11) will flow straight into the tissue in which a certain amount is dissipated according to Eq. (2.22). An SAR and temperature hotspot is formed at these locations.

\subsubsection{Wire surrounded by spacer}

In the second scenario in which a spacer is surrounding the wire, the SAR will be lower in adjacent tissue for two reasons. When we consider a non conductive spacer material, the conduction currents running from gel to spacer will be non existent in the spacer following

$$
\boldsymbol{J}_{\text {spacer }}^{c}=\sigma_{\text {spacer }} \boldsymbol{E}_{\text {spacer }} .
$$

The conduction current in the spacer is zero for a non-conducting material. Now, because the conduction current from the tissue towards the wire is blocked, this leaves the wire with less power to channel towards the tips. The electric fields that do reach the wire will form a conduction current as in the first scenario in the direction over the wire. However, the spacer material at the wire tip has zero conductivity preventing the current from flowing into the gel at the tips. As a result, the energy will be contained in the wire spacer system, storing charge on the wire similar to a capacitor. Overall the SAR will be lower around the wire tips compared to the bare wire scenario. 


\subsubsection{Wire partially surrounded by spacer}

A scenario in which the retainer wire produces a high SAR level is the case of a 'leaking' spacer. The worst case scenario describes an opening in the spacer at both wire tips through which conductive material connects wire and tissue. Conduction currents of surrounding tissue channel will flow in and out of these wire tips. This channel will lead to very localized intense heating as described by Nyenhuis [19] for an insulated wire with bare tips. This leaky spacer is worse in terms of SAR compared to a bare wire, since part of the currents over the bare wire will flow into the tissue before reaching the tips.

\subsubsection{High E field in the spacer}

An effect to be expected due to the low conductivity and low permittivity of the spacer is a higher electric field in the spacer compared to the surrounding tissue. In simulations this can appear to look like a simulation error. The higher E field is not harmful since it only resides in the spacer. The effect can be described through the boundary conditions of the tissue-spacer boundary, in cylindrical coordinates $\left(\boldsymbol{i}_{r}, \boldsymbol{i}_{\theta}, \boldsymbol{i}_{z}^{\prime}\right)$. We consider the cylindrical wire to be extending to infinity and having radius $r_{\text {wire }}$, the wire is surrounded by a spacer equally extending to infinity having outer radius $r_{\text {spacer }}$. The spacer is surrounded by tissue, which is extending to infinity in all directions.

The normal components of the electric flux density to the spacer-tissue interface are continuous when traversing this boundary. In cylindrical coordinates $\left(\boldsymbol{i}_{r}, \boldsymbol{i}_{\theta}, \boldsymbol{i}_{z}\right)$, the normal component to the the spacer-tissue boundary is the radial component. The two media have different electric properties, the normal component of the electric field will therefore have a jump over the boundary. The tangential components of the electric field are continuous over the interface and do not exhibit a jump in the electric field strength. The tangential components in cylindrical coordinates are the components in $\theta$ and $z$-direction. Hence, for the radial component of the electric flux density across the spacer interface we have

$$
\begin{aligned}
J_{\text {radial }} & =\sigma E_{\text {radial }}+j \omega \epsilon E_{\text {radial }} \\
\lim _{r \uparrow r_{\text {spacer }}} J_{\text {radial }}^{(\text {spacer })} & =\lim _{r \downarrow r_{\text {spacer }}} J_{\text {radial }}^{(\text {tissue })}
\end{aligned}
$$

The $\mathrm{E}$ field at one side of the boundary can quantitatively be described in terms of the $\mathrm{E}$ field at the other side of the boundary together with the conductivity and permittivity of both media:

$$
\lim _{r \uparrow_{\text {spacer }}} E_{\text {radial }}^{(\text {spacer })}=\frac{\sigma_{(\text {tissue })}+j \omega \epsilon_{(\text {tissue })}}{\sigma_{(\text {spacer })}+j \omega \epsilon_{(\text {spacer })}} \lim _{r \downarrow r_{\text {spacer }}} E_{\text {radial }}^{(\text {tissue })}
$$

For the previously described zero conductivity spacer $\sigma_{\text {spacer }}=0$ Eq. (4.13) reduces to

$$
\begin{gathered}
\lim _{r \uparrow r_{\text {spacer }}} E_{\text {radial }}^{(\text {spacer })}=\left[-j \frac{\sigma_{(\text {tissue })}}{\omega \epsilon_{\text {spacer }}}+\frac{\epsilon_{(\text {tissue })}}{\epsilon_{(\text {spacer })}}\right] \lim _{r \uparrow r_{\text {spacer }}} E_{\text {radial }}^{(\text {tissue })} \\
\lim _{r \uparrow r_{\text {spacer }}}\left|E_{\text {radial }}^{(\text {spacer })}\right|=\left|-j \frac{\sigma_{(\text {tissue })}}{\omega \epsilon_{\text {spacer }}}+\frac{\epsilon_{(\text {tissue })}}{\epsilon_{(\text {spacer })}}\right| \lim _{r \uparrow r_{\text {spacer }}}\left|E_{\text {radial }}^{(\text {tissue })}\right|
\end{gathered}
$$

In which the described E field components are complex valued quantities. When the values for the phantom simulation are filled in for this equation, the ratio between the radial components 
of both electric fields can be shown to be 206 .

$$
\lim _{r \uparrow r_{\text {spacer }}}\left|E_{\text {radial }}^{(\text {spacer })}\right|=206 \lim _{r \uparrow r_{\text {spacer }}}\left|E_{\text {radial }}^{(\text {tissue })}\right|
$$

This is however no cause for heating, since the spacer is not conductive. All of this electric energy is stored and not dissipated in the spacer as described in Eq. 2.18 by the second and third term. The behavior of the $\boldsymbol{E}$ fields in the spacer is illustrated in Section 6.2, Fig. 6.5b.

\subsection{Concluding remarks on tissue heating around a wire}

Any bare metal wire inside tissue will have most of its SAR concentrated at the wire tips. A wire with length around 0.5 times the wavelength is expected to have the highest SAR since the currents over the wire will be in resonance. The increase in SAR is to be verified in simulations and temperature measurements.

In case the SAR limits are exceeded for the bare wire, we propose the use of a spacer around the wire. The spacer not only thermally shields the tissue from the wire, the conducting currents channeled at the wire tips potentially flowing between the tissue and wire are reduced or even canceled. This effect takes down the source to the tissue heating problem. The energy of the electric fields will due to a low conductivity spacer be largely stored in the wire-spacer system without dissipating the energy as described in the compex Poynting theorem in Eq. (2.18). This spacer will have a lower SAR as a result, simulations will have to quantify the order of SAR reduction due to a spacer around the wire.

Measurement and simulation results of a wire with spacer, in this case a heat shrink tube, are provided in chapter 5. Additional simulation results for a set of wire spacers with different thicknesses in a phantom are described in chapter 6 . 


\section{Chapter 5}

\section{Temperature simulations and measurements}

A magnetic deflection test is performed on the available retainer wires to assess the attraction force by the main static magnetic field $\left(\boldsymbol{B}_{0}\right)$. The MR magnets attraction force to the wire is defined in terms of greater than or smaller than the force applied to the wire by the Earths magnetic field.

Thermal measurements are performed to validate electromagnetic and thermal simulations of the phantom setup. Temperature measurements are performed using a set of three fluor optic measurement probes (Opsens technology) on a set of dental retainer wires (nickel free stainless steel) in a phantom at 7 tesla during a SAR intensive scan. The wire lengths used are $20 \mathrm{~mm}$ to $70 \mathrm{~mm}$. The measurements also include a wire with spacer to verify the SAR reduction as described in the previous chapter. The simulations are scaled to the MRI power settings for comparison. The method used to link both powers is described in Section 5.2.

\subsection{Magnetic deflection test}

A dozen retainer wires from several vendors have been subjected to a magnetic deflection test. The test, performed according to the ASTM F2052 standard, [22], assesses the maximum magnetically induced displacement force applied to the wire for a patient going into the scanner. The outcome of the test indicates that the force to the wire will either be larger or smaller than the force applied to the wire by the Earth magnetic field. For comparison, the gravitational field applies a force of about $0.01 \mathrm{~N}$ to a typical stainless steel $50 \mathrm{~mm}$. The range of wire materials includes stainless steel, some are nickel free while others contain nickel which is highly magnetic and a single one is made of titanium.

\subsubsection{Magnetic deflection test setup}

The test apparatus consists of a nonmagnetic structure that is strong enough to withstand the deflection forces during the test and holds a protractor. The end of a string is fixed to the device under test (DUT) and the other end is fixed to the center of the protractor, such that the string angle is indicating 0 degrees when placed outside the MR room. The string is chosen as light as possible not to influence the measurement. 
The setup is positioned at the entrance of the MR bore at the location of maximum flux density $\boldsymbol{B}_{\mathbf{0}}$. The length of the string is chosen such that the DUT is suspended halfway the height of the bore. The force applied to the retainer wire by the $\boldsymbol{B}_{\mathbf{0}}$ field is purely horizontal, the earth gravitational field is purely vertical. The combined force of the MR magnet and the earth gravitational field will have the wire pointing at an angle $\theta$. An angle $\theta$ smaller than $45^{\circ}$ will indicate an MR force to the wire smaller than the earths magnetic field, an angle larger than $45^{\circ}$ indicates a larger force.

\subsubsection{Magnetic deflection test results and discussion}

Wires with ID \# 3, 8, 10 and 12 have a measurable force to them which is between 0 and 90 degrees. Wire \#11 is not attracted, while all the others exceed the maximally quantifiable force e.g. measure an angle equal to or greater than 90 degrees. The latter does not directly mean they are not safe since retainer wires are sturdily glued to the teeth. However, a person with such wire could feel some attraction of the wire, for wires measuring more than $45^{\circ}$ indicate that the magnetic attraction force is larger than the force of the earths magnetic field to that wire. All magnetic deflection results are summarized below

Table 5.1: Magnetic wire deflection test results

\begin{tabular}{|c|c|c|c|c|}
\hline ID & Wire name & Length mm & weight $g$ & angle \\
\hline 1 & Quadcat $016 \times 22$ & 110 & 0.15 & $>90^{\circ}$ \\
\hline 2 & Dentaflex 020 & 100 & 0.12 & $>90^{\circ}$ \\
\hline 3 & Solid wire 016 x 22 & 105 & 0.21 & $52^{\circ}$ \\
\hline 4 & Pentacat 0215 & 93 & 0.14 & $>90^{\circ}$ \\
\hline 5 & Forestaflex 016 x 0016 F200-2240 & 60 & 0.06 & $>90^{\circ}$ \\
\hline 6 & Penta One. 0215 Ma49982 & 60 & 0.09 & $>90^{\circ}$ \\
\hline 7 & Pentacat.0215 gac $03-215-28$ & 63 & 0.09 & $>90^{\circ}$ \\
\hline 8 & Titan .020 (nickel free) D528-000-00 & 50 & 0.03 & $5^{\circ}$ \\
\hline 9 & Romanium .018 Dentaurum D535-045-0 & 64 & 0.0 & $>90^{\circ}$ \\
\hline 10 & Noninium (nickel free) 0.018 Dentaurum D764-003-00 & 66 & 0.09 & $19^{\circ}$ \\
\hline 11 & Ortho Flex Tech Dental retention system Ref\#0FT2 & 24 & 0.08 & $0^{\circ}$ \\
\hline 12 & Wildcat & 70 & 0.07 & $20^{\circ}$ \\
\hline
\end{tabular}

The wire used in the temperature measurements is \#12 in Table 5.1. The wire did not show any bending due the magnetic fields attraction force in the temperature measurement setup, which could influence the temperature registration.

\subsection{MRI simulations and measurements}

\subsubsection{Temperature measurements methods and test setup}

The main goal of the temperature measurements is to validate the EM simulations and thermal simulations of the phantom setup. The temperature measurements are as far as possible performed in accordance to the ASTM standard test method, ASTM F2182-11a for measurement of radio frequency induced heating on or near passive implants during magnetic resonance imaging. A set of wires with different lengths in the range of 0 to $70 \mathrm{~mm}$ has been tested. From which we obtain a SAR level for each wire length. Together with the simulations in the CST microwave 
studio environment as described in Section 5.2.2 we can make a validation of the simulation results.

The measurement setup is build up around a cylindrical phantom which has dimensions of 176 $\mathrm{mm}$ in diameter and $220 \mathrm{~mm}$ in length, filled with a saline Hydroxy Ethyl Cellulose gel mixture according to the ASTM standard. The electrical properties of the gel are a relative permittivity of 81 and conductivity at $298.2 \mathrm{MHz}$ of $0.47 \mathrm{~S} / \mathrm{m}$. The retainer wire under testing is positioned half way into the phantom and at a distance $15 \mathrm{~mm}$ from the cylindrical wall as can be seen in the simulation model of Fig. 5.1a and in the image of the real phantom in Fig. 5.1b. The retainer wire used in the experiments is the 'Wildcat' stainless steel three strand twisted wire measuring $0.55 \mathrm{~mm}$ in diameter. Five lengths are tested, $20 \mathrm{~mm}, 35 \mathrm{~mm}, 47.5 \mathrm{~mm}, 55 \mathrm{~mm}$ and $70 \mathrm{~mm}$. In addition two tests are performed, one with a $47.5 \mathrm{~mm}$ wire with spacer surrounding the full wire and the second with the phantom without a wire as a baseline for the the other measurements. All tests are performed three times.

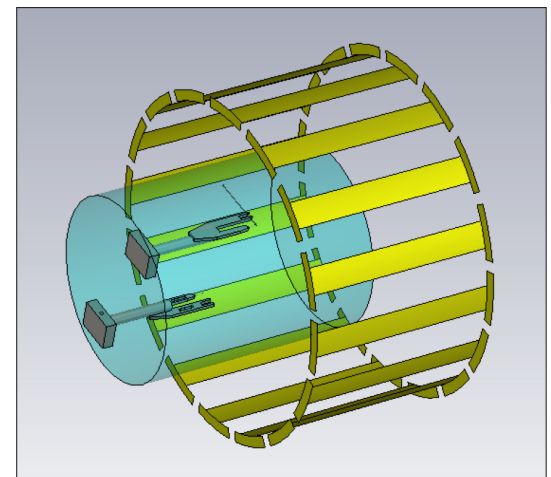

(a) Simulation model setup

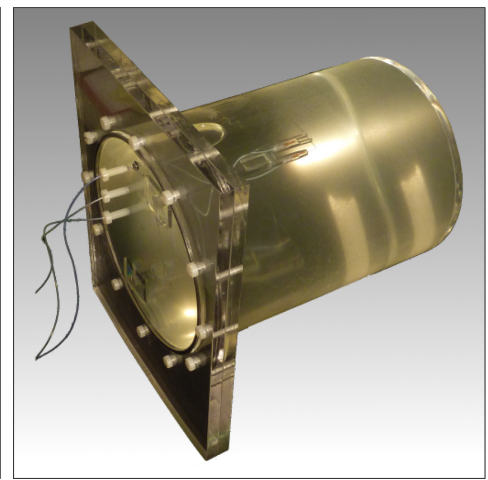

(b) MRI phantom

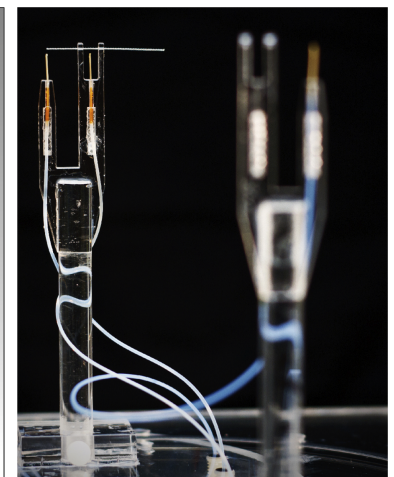

(c) Measurement platform with straight wire

Figure 5.1: Measurement setup

The temperature measurements in the phantom are performed using three fluor optic temperature probes with accuracy: $0.3{ }^{\circ} \mathrm{C}$ and resolution $0.1{ }^{\circ} \mathrm{C}$ and logged using the vendor supplied software and hardware. The placement of the sensors is at $1.5 \mathrm{~mm}$ from wire tip, $1.5 \mathrm{~mm}$ from the wire center and one as reference on opposite side of the phantom symmetrical to the tip sensor. The heating MR sequence takes 15 minutes in according to the ASTM standard, during which the temperature change is constantly monitored at a sample interval of 2.8 seconds. Three consecutive measurement-sets are performed for each wire setup and at least 15 minutes were allowed between successive sets to allow re-establishment of thermal equilibrium at the sensors.

A spacer around the wire as described in the simulations Section 6.2.1 is made using a heatshrink tube having almost identical electrical properties compared to air. Heat shrink conductivity at $298 \mathrm{MHz}$ is about $10^{-14} \mathrm{~S} / \mathrm{m}$ and its relative permittivity is 2, compared to air $0 \mathrm{~S} / \mathrm{m}$ and relative permittivity of 1 . Measurements on a wire with the spacer are performed on a single wire length of $47.5 \mathrm{~mm}$.

A multi slice turbo spin-echo imaging sequence was used with the scanner SAR safety limit over-ridden in software. Transmit power levels between 31 and 83 watts continuous were used for a power sweep on the $56 \mathrm{~mm}$ wire, while the full set of wire lengths has been scanned at a fixed power level of $70 \mathrm{~W}$. The parameters of the multislice turbo spin echo (MS TSE) sequence are chosen such that the 'imaging time' is 15 minutes by setting the number of signal averages 
(NSA) to 19 in combination with a repetition time (TR) of $657 \mathrm{~ms}$. The other scan parameters are: an echo time (TE) is set at $26 \mathrm{~ms}$, the acquiring matrix is $312 \times 304$ with a field of view (FOV) of $250 \times 250 \times 52$.

\subsubsection{Electromagnetic simulation methods}

The electromagnetic simulations are performed at the $7 \mathrm{~T}$ frequency of $298.2 \mathrm{MHz}$, using the finite difference time domain (FDTD) solver CST microwave studio. An FDTD solver is selected for it is able to handle voxelized models and the combination of metal and dielectrics well. A 16-leg high pass birdcage head coil with length $180 \mathrm{~mm}$, diameter $300 \mathrm{~mm}$, is used driven by 32 discrete sources as depicted in Fig. 5.2

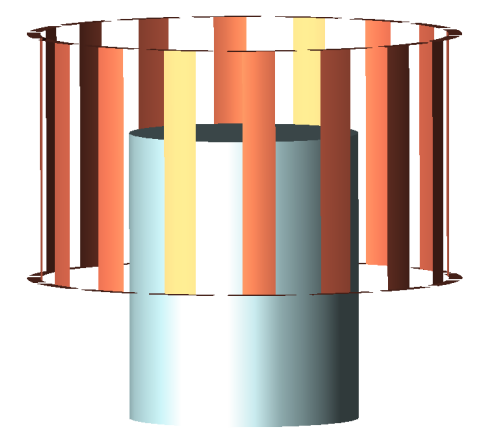

Figure 5.2: Homogeneous cylindrical phantom in $7 \mathrm{~T}$ birdcage head coil.

In the figure we see the 16 vertical birdcage legs ended on both sides with an end ring. The sources are feeding in between every segment on the end-rings, up to a total of 32 feeds. These are driven simultaneously on $298.2 \mathrm{MHz}$ with a phase difference of $360 / 16=22.5^{\circ}$ between consecutive sources on the rings to create a rotating $\boldsymbol{B}_{1}$ field. During simulations the $\boldsymbol{E}$ field and related voxel-based local SAR and SAR $1 g$ values are acquired together with the $\boldsymbol{H}$ fields and related $\mathrm{B}_{1}^{+}$and $\mathrm{B}_{1}^{-}$. The average power going into the head coil is set equal to the power going into the headcoil of the real MR setup as described in the following section.

\subsubsection{Linking MR transmitted power to simulated transmitted power}

For the investigation of the accuracy of the SAR simulations we want to match the power going into the simulated birdcage coil to the power going into the coil of the MR scanner. The amount of power going into the simulated coil is provided in the simulations. However, the power going into the MR coil is not directly measured by the scanner, which demands for a work-around. 


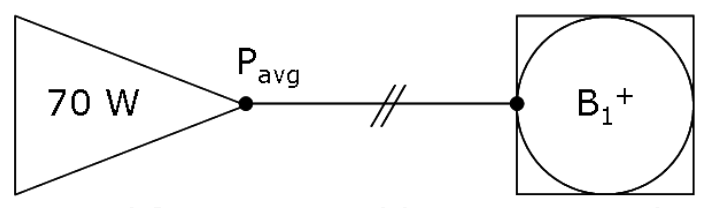

Amplifier
Cable

(a) MR transmitted power, $P_{a v g}$

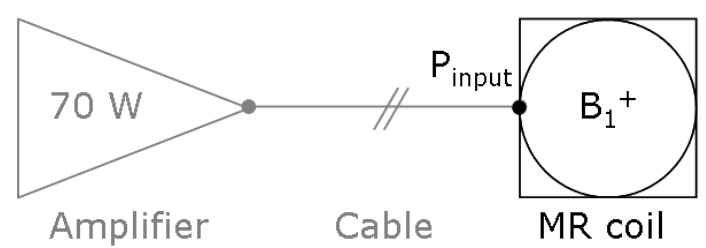

(b) Simulated input power, $P_{\text {input }}$

Figure 5.3: Linking the average transmit power, going into the coil of the MR scanner, and going into the simulated coil. The power going into the MR coil is not directly measurable

The figures show schematically at which locations the transmit powers are known for the simulation and scanner. The MR scanner power is measured at the amplifier output, not at the transmit coil itself, while the transmitted power in the simulations is defined at the feed points of the transmit coil. The difference between both powers is about $50 \%$ due to losses in the cable connecting the amplifier to the birdcage coil. What we need in order to compare the input powers of the MR coil and simulation coil is a separate parameter which is available in both measurement and simulation.

A common ground to link both setups is the $\mathrm{B}_{1}^{+}$, which is measureable in 3 dimensions in the scanner and is provided in the simulations. We assume that both coil/phantom setups behave the same and therefore generate equal $\mathrm{B}_{1}^{+}$distributions in the phantom of the simulation and in the real setup. A transmit efficiency, relating $\mathrm{B}_{1}^{+}$to average input power, has been defined for the purpose of linking MR simulations and MR measurements and is used by Teeuwisse. et al. [26].

$$
\text { Transmit efficiency }=\frac{\boldsymbol{B}_{1}^{+}}{\sqrt{P_{\text {transmitted }}}}(\mu T / \sqrt{W})
$$

The MR scanner provides the time-averaged transmitted power to the MR coil, minus the reflected power, at its RF amplifiers which is used for the transmit efficiency. The input power to the simulations can now be related to the MR transmit power through the ratio of both transmit efficiencies.

$$
P_{\text {input simulation }}=\left[\frac{\text { Transmit efficiency MR scanner }}{\text { Transmit efficiency simulation }}\right]^{2} P_{\text {scanner average }}
$$

This relation provides means to compare the SAR in the simulations to the SAR during a heating sequence. In which the SAR in the heating sequence is calculated through SAR calorimetry as described in Section 4.2.1.

\subsection{4 $\quad \mathrm{B}_{1}^{+}$Measurement and transmit efficiency}

Measurement of the $\mathrm{B}_{1}^{+}$in the phantom due to excitation of the MR coil is possible through a 3D flip-angle measurement technique, by Yarnykh [25]. The acquired flip angles at each position in the phantom can henceforth be recalculated to $\mathrm{B}_{1}^{+}$using Eq. (2.11). The flip-angle sequence is based on the principle in which the MR signal is measured at two different repetition times after an equal excitation pulse. The flip angle can through the flip-angle sequence be calculated from the ratio of both received signals.

The transmit efficiency is calculated from the obtained $\mathrm{B}_{1}^{+}$value, for a homogeneous region of maximum strength in the phantom, in combination with the average transmit power at the 
MR amplifier. The calculated efficiency of this phantom setup is valid for all MR sequences. This includes the SAR intensive 'heating sequence', which is played out during the temperature measurements. The results of the $B_{1}$ mapping, together with the actual transmit power define a transmit efficiency of $0.58 \mu \mathrm{T} / \sqrt{W}$.

The efficiency of the coil in the simulation in terms of $\mathrm{B}_{1}^{+}$over square root of power is obtained for a simulation at $298.2 \mathrm{MHz}$. The maximum efficiency is $0.78 \mu \mathrm{T} / \sqrt{W}$ in the same region as in the MR measurement.

The input power to the simulated coil is equal to the ratio, Eq (5.2), of both transmit efficiencies squared times the average transmitted MR power, which is $0.553 \cdot \mathrm{P}_{M R}$. This means that a time average input power of $70 \mathrm{~W}$ for the $\mathrm{MR}$ scan is equal to $38.7 \mathrm{~W}$ in the simulation. As summarized in the following table

Table 5.2: Power linking

\begin{tabular}{l|l|l} 
& $\mathrm{B}_{1}^{+} / \sqrt{P}$ & Power for heating sequence \\
\hline \hline MR scanner & $0.58 \mu \mathrm{T} / \sqrt{(\mathrm{W})}$ & $70 \mathrm{~W}$ \\
Simulation CST & $0.78 \mu \mathrm{T} / \sqrt{(\mathrm{W})}$ & $38.7 \mathrm{~W}$
\end{tabular}

These values are used as the basis for the simulations and MR heating experiments.

\subsection{Measurements and simulation results}

\subsubsection{Measured and simulated temperature rise: one wire}

Temperature measurements are performed on the phantom to verify simulation methods and results on the phantom and on a human head model. CST's electromagnetic solver, "microwave studio", is used to provide a 3D map of dissipated power in the phantom, showing the location of maximum SAR . The time-average power going into the coil, $38.7 \mathrm{~W}$, is matched to the $70 \mathrm{~W}$ average power going into the real MR coil during the wire heating experiments in the MR scanner. From the simulation results of the dissipated power we calculate the consecutive temperature rise in the phantom using CST's multiphysics studio software. Three temperature probes, as described in the measurement method Section 5.2.1, are positioned at the same locations in the simulated and real phantom. A typical retainer wire is $35 \mathrm{~mm}$ in length. For which the simulated and measured temperature data of the three sensors during the 15 minutes of heating are depicted below for comparison. This is data from only one measurement, the data in later sections is averaged over three measurements. 


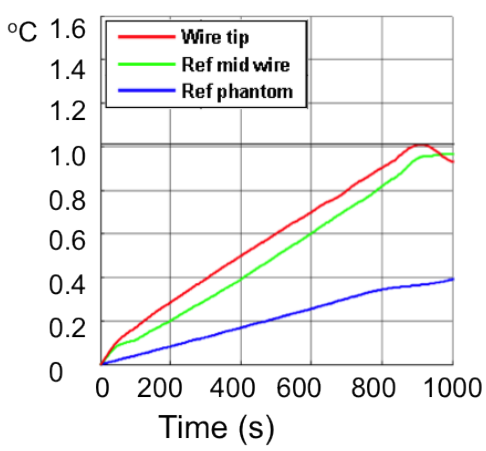

(a) Measured temperature rise

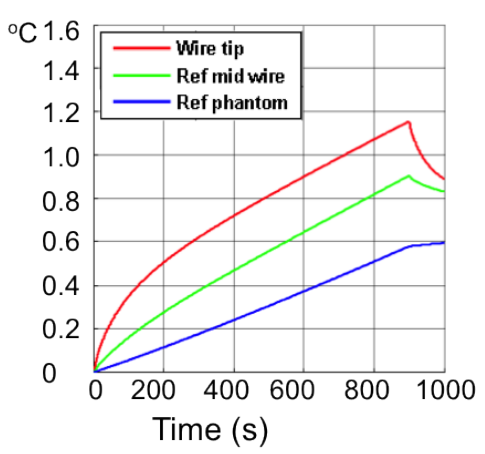

(b) Simulated temperature rise

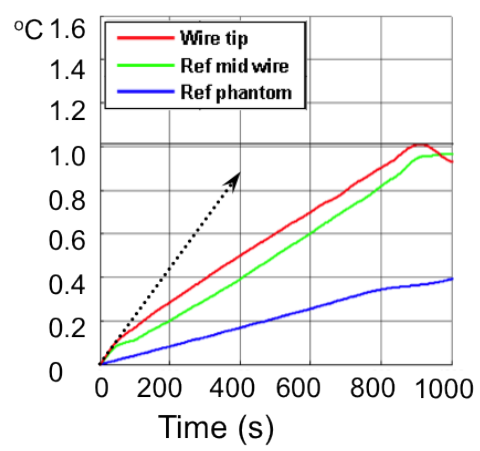

(c) Measured temperature: SAR calculation from temperature slope

Figure 5.4: Measured and simulated temperature rise around $35 \mathrm{~mm}$ wire. During $900 \mathrm{sec}$ heating sequence at $70 \mathrm{~W}$ average MRI power

The three curves in each graph represent the temperature increase measured at, in ascending order of magnitude, the reference sensor, mid-wire sensor and wire-tip sensor. The temperature rise in both figures is the highest for the tip, out of the three sensor locations. The maximum measured temperature rise for the $35 \mathrm{~mm}$ wire in this single measurement is $1.0^{\circ} \mathrm{C}$. The $\mathrm{RF}$ source is switched off at $\mathrm{t}=900 \mathrm{~s}$ (15 minutes) after which we see a steep cooling effect at the wire tip, making its way back to thermal equilibrium. Furthermore it is noted that the increase in tip temperature is more rapid in the first 20 seconds compared to the later stages. The thermal inertia is causing a delay in heat diffusion throughout the surrounding tissue at the early stage of heating. The temperature slope in the initial phase as depicted in Fig. 5.4c is used for the SAR calculations. The curve of the simulated temperature rise is slightly more rapid in its growth, yet has its maximum temperature of $1.1^{\circ} \mathrm{C}$ fairly close the measurement maximum, with a difference of $0.1^{\circ} \mathrm{C}$.

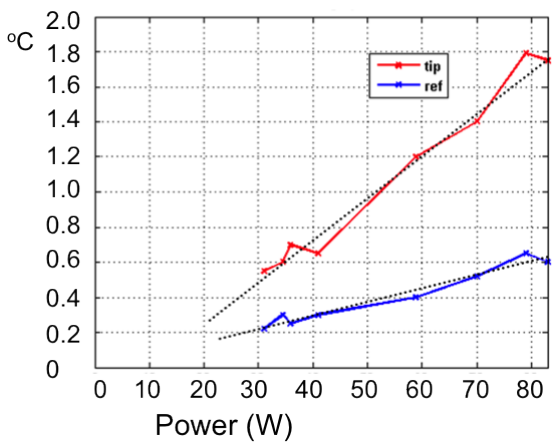

Figure 5.5: Measured temperature rise after $900 \mathrm{sec}$ heating. For various input powers. 47.5 $\mathrm{mm}$ wire

The measured temperature rise after 900 seconds of heating, increases linearly with input power for the measured power range between $35 \mathrm{~W}$ and $83 \mathrm{~W}$. 


\subsubsection{Measured and simulated temperature: wires 0 to $70 \mathrm{~mm}$}

Resonance of the current over the wire can boost the SAR and temperature rise in the surrounding tissue. As a rule of thumb resonance takes place at the half wave length, as discussed in section 4.1. For the phantom as described at the beginning of this chapter, having a relative permittivity 81 , conductivity $0.47 \mathrm{~S} / \mathrm{m}$ and relative permeability of 1 , the resulting half wavelength is $55 \mathrm{~mm}$ following Eq. (4.7). Wires in the range of 0 to $70 \mathrm{~mm}$ are assessed in phantom simulations and temperature measurements in the scanner. This will provide the resonant wire length and its corresponding increase in both SAR and temperature rise. The results of the wire length sweep will also indicate how narrow or wide the resonance peak is.
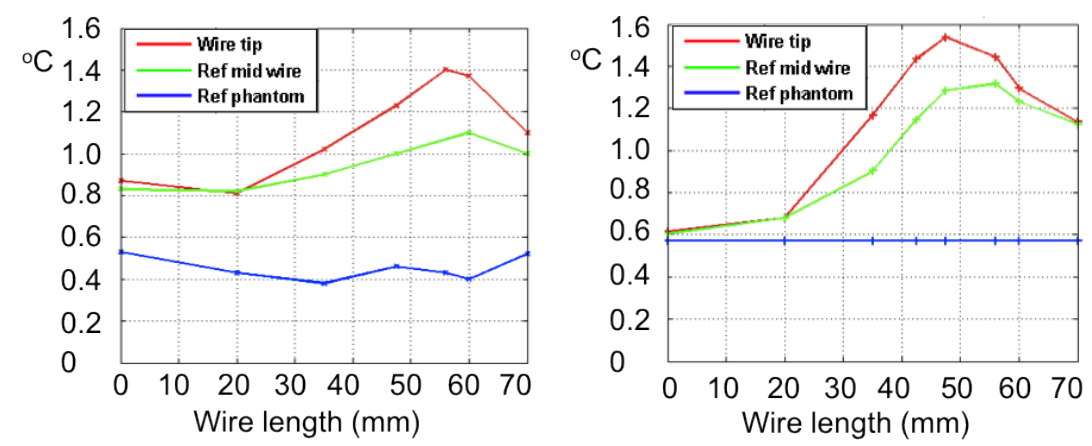

(a) Measured temperature rise after 15(b) Simulated temperature rise after 15 minute scan. $70 \mathrm{~W}$ average power minutes

Figure 5.6: Measured and simulated temperature rise at the wire tip, mid wire and at reference probe after 15 minute $\mathrm{RF}$ excitation for $\mathrm{P}_{\mathrm{MR}}=70 \mathrm{~W}$. For wires of $0 \mathrm{~mm}$ to $70 \mathrm{~mm}$ in length

The maximal temperature rise after 15 minutes is recorded for all wires in the range of 0 to 70 $\mathrm{mm}$. The top figure is showing the wirelength vs. temperature rise in ${ }^{\circ} \mathrm{C}$ for both measurements and simulations. The measurements are depicted in the bottom left figure and the simulations in the bottom right figure. The measured temperatures, in the graph on the left, show for the wire-tip the highest temperature rise with a maximum of $1.4^{\circ} \mathrm{C}$. The tip sensor for the experiment without a wire ('0 mm'), shows a rise of $0.8^{\circ} \mathrm{C}$. This means that the baseline SAR in the phantom is already high enough to heat the gel, also in absence of a metal implant. If we compare the ' $0 \mathrm{~mm}$ ' tip-sensor to the reference sensor, which is on the other side of the phantom, one would expect the same rise in temperature since both sensors are at equal distance from the cylinder wall and therefore at equal distance from the coil. Their difference, $0.5^{\circ} \mathrm{C}$ (reference) and $0.8^{\circ} \mathrm{C}$ (tip sensor) is however not very large, so it appears to be an error in the measurement, either due to the sensor accuracy $\left(0.3^{\circ} \mathrm{C}\right)$, sensor placement accuracy or residual heat diffusion after the previous measurement. The temperature rise measured by the reference sensor does not show a constant behaviour over the range of wires from 0 to $70 \mathrm{~mm}$ as would ideally be expected. The deviation is however only $0.1{ }^{\circ} \mathrm{C}$.

The maximum simulated temperature rise after 15 minutes of $\mathrm{RF}$ excitation is $1.4^{\circ} \mathrm{C}$ at a wire length of $47.5 \mathrm{~mm}$. Which is above the IEC temperature limit of $1^{\circ} \mathrm{C}$, however, since the average power going into the scanner exceeds SAR safety settings by a factor of 5 , this would be no problem in terms of temperature rise when the lower normal power setting is used. We have seen that temperature rise increases linearly with power for a limited range of input powers. This relation is illustrated in Fig. 5.5 of measurements on the MR scanner for average powers 
in the range of $35 \mathrm{~W}$ to $83 \mathrm{~W}$. If the power setting of 70 watts is reduced to 35 watts then the resulting temperature rise at the tip is only a $0.6^{\circ} \mathrm{Cwhich}$ is below the temperature limit.

The short wire of $20 \mathrm{~mm}\left(0.6^{\circ} \mathrm{C}\right)$ has only a minimal gain in temperature rise compared to the 'no wire' case $\left(0.55^{\circ} \mathrm{C}\right)$. Wires longer than the resonant length still show a relatively high temperature rise. Wires longer than $70 \mathrm{~mm}$ are not taken into account, since they are not used as a retainer wire.

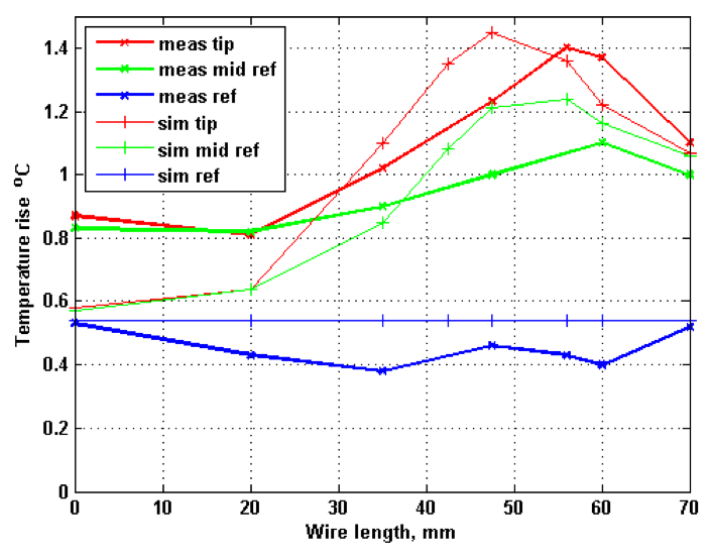

Figure 5.7: Combined measured and simulated temperature rise for wires 0 to $70 \mathrm{~mm}$

If we compare the measurement results to the simulations, we see that the maximum temperature rise is almost equal with only a $0.05{ }^{\circ} \mathrm{C}$ difference. Although the resonance length is shifted slightly, $56 \mathrm{~mm}$ for the measurement and $47.5 \mathrm{~mm}$ in simulations. Overall, the temperature measurements agree well with the simulated temperatures after the 15 minutes of heating. SAR calculation and comparison is the following step to assess the wires for RF safety compliance.

\subsubsection{Measured and simulated SAR: wires 0 to $70 \mathrm{~mm}$}

SAR calculation is performed for both simulations and temperature measurements at the same location at the wire tip for equal comparison. The calculation is performed as described in the SAR calorimetry section (4.2.1) and illustrated in Fig. 5.4c. The method uses the difference in temperature during a short interval at the beginning of excitation to calculate the dissipated power. The temperature curves of the measurements and simulations of the wires of 0 to $70 \mathrm{~mm}$ are all converted to a local SAR calculated value. The calculated SAR in the simulations appeared to be close to the 1 gram SAR, although we will use the $\mathrm{SAR}_{\text {calculated values only to compare }}$ the results of the measurements to the simulations. 


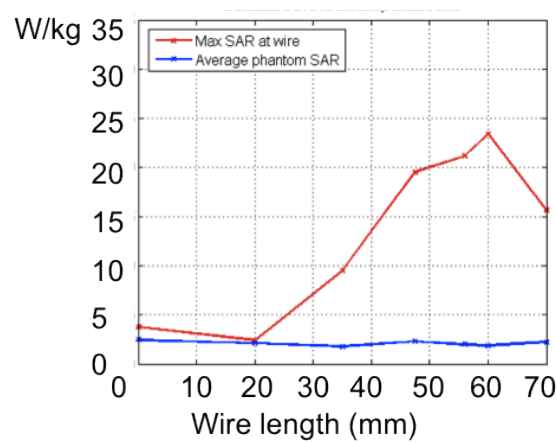

(a) Calculated SAR, temperature measurements

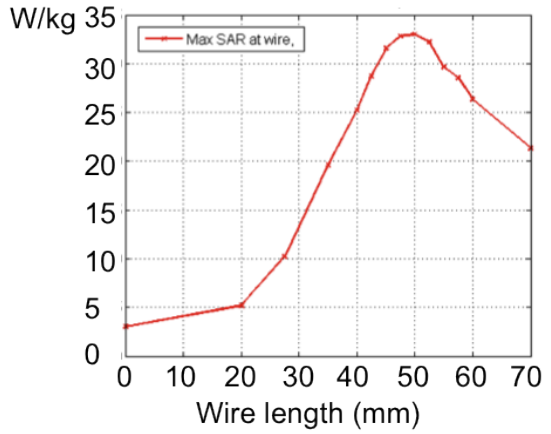

(b) Calculated SAR from simulation

Figure 5.8: Calculated SAR around the wire from temperature measurements and simulations

From the simulated temperature rise results we calculate the SAR in the simulations, using the same method as for the thermal measurements for fair comparison between both sets. The SAR results for the range of wires, in Fig. 5.8b, presents a maximum SAR of $32 \mathrm{~W} / \mathrm{kg}$ which is 10 times higher than having no wire at all. The wire with maximum SAR is found to be at $50 \mathrm{~mm}$ which is reasonably close to the theoretical half wavelength. The SAR in terms of wire length is lowest for short wires, and peaks at resonance. The SAR calculated from the temperature measurements has its peak of $24 \mathrm{~W} / \mathrm{kg}$ at $60 \mathrm{~mm}$. The noise in the temperature measurements make a good registration of the start and end point of the time interval difficult to use in the calculations. A low pass filter is applied over the measured data for SAR calculation, which resulted in a smoother curve and more realistic SAR results. Data extraction still remained slightly ambiguous, which explains why the SAR graph looks less smooth compared to the simulated figure.

An overview of the calculated SAR values from the graphs of Fig. 5.8 are given for ease of comparison between maxima, minima and to having no wire at all.

Table 5.3: Wire with maximum and minimum $\mathrm{SAR}_{\text {calculated, }}$ measured and simulated.

\begin{tabular}{l|l|l|l|l}
$\begin{array}{l}\mathrm{SAR}_{\text {calculated }} \\
{[\mathrm{W} / \mathrm{kg}]}\end{array}$ & no wire & $\begin{array}{l}\min \mathrm{SAR} \\
22 \mathrm{~mm}\end{array}$ & $\begin{array}{l}\max \mathrm{SAR} \\
50 \mathrm{~mm}\end{array}$ & $\begin{array}{l}\max \mathrm{SAR} \\
60 \mathrm{~mm}\end{array}$ \\
\hline Measured & 4 & 3 & 20 & $\mathbf{2 4}$ \\
Simulated & 3.2 & 5.0 & $\mathbf{3 2}$ & 26
\end{tabular}

When we compare the measurement-SAR to the simulated-SAR over the range of wire lengths, the most obvious difference is the maximum SAR value. We compare the maximum SAR values for the measurements and the simulations. The maxima in both sets are $24 \mathrm{~W} / \mathrm{kg}$ versus $32 \mathrm{~W} / \mathrm{kg}$ respectively for measurements and simulations. An explanation for their difference could be the noise over the measured temperature data. The input powers have been scaled by a factor of 5 . A normal scan would have $1 / 5$ th of the power, which for the simulated maximum is $6.4 \mathrm{~W} / \mathrm{kg}$. Which is just below the IEC SAR $1 g$ norm of $8 \mathrm{~W} / \mathrm{kg}$. We note that, while the $\mathrm{SAR}_{\text {calculated }}$ at the resonant wire is 10 times higher than the background $\operatorname{SAR}_{\text {calculated }}(3.2 \mathrm{~W} / \mathrm{kg})$, smaller wires up to $65 \%$ of the resonant length have only half the maximum $\mathrm{SAR}_{\text {calculated }}$ or less. 


\subsubsection{Wire with spacer}

Temperature simulations and measurements are performed on a single $47.5 \mathrm{~mm}$ wire with spacer. The simulated spacer properties are chosen equal to air, so we have $\epsilon=\epsilon_{0}, \mu=\mu_{0}$ and conductivity $\sigma=0$. The wire enclosing layer is $0.38 \mathrm{~mm}$, such that in combination with $0.55 \mathrm{~mm}$ wire diameter we have a total diameter of $1.3 \mathrm{~mm}$. The spacer extends $1 \mathrm{~mm}$ at both ends over the wire, surrounding it completely without any gaps. The result after a 15 minute heating session in terms of temperature rise is: $0.9{ }^{\circ} \mathrm{C}$ with a SAR of $4 \mathrm{~W} / \mathrm{kg}$. Which is close to having no wire at all, and having SAR lower than the $20 \mathrm{~mm}$ wire.

A single wire with spacer is tested in the temperature measurement as well setup. The spacer length and thickness $(0.5 \mathrm{~mm})$ is equal to the spacer described in the previous simulation section. The spacer material of choice is a heat-shrink tube, having permittivity $\epsilon=2, \mu=\mu_{0}$ and conductivity $\sigma=10^{-8} \mathrm{~S} / \mathrm{m}$. The ends of the tube are sealed with hot glue, having similar electrical properties as the tube, such that there is not connection between the saline gel and the metal wire. The temperature measurement sensor at the tip, at the same distance from the metal wire as before, measured a maximal increase in temperature of $0.75^{\circ} \mathrm{C}$. This is after a 15 minute heating scan, repeated three times with enough time in between to re-establish thermal equilibrium at the measurement sensors. The temperature results for the phantom without wire provided $0.8^{\circ} \mathrm{C}$. Concluding, this spacer reduces the SAR around the wire to an extend that surrounding tissue is not heated by the wire at all.

Table 5.4: Wire with spacer, Measured and simulated. Calculated $\mathrm{SAR}_{1 \mathrm{~g}}$ at wire tip. $70 \mathrm{~W}$ MRI average power

\begin{tabular}{l|l|l|l} 
& no wire $[\mathrm{W} / \mathrm{kg}]$ & with spacer $[\mathrm{W} / \mathrm{kg}]$ & Bare wire $[\mathrm{W} / \mathrm{kg}]$ \\
\hline Measured & 3.7 & 3.3 & 19.2 \\
Simulated & 3.8 & 3.6 & 32
\end{tabular}

\subsubsection{Discussion on simulated and measured SAR and temperature rise}

This study aims to find the highest SAR configurations of a retainer wire. The practical retainer wire would fit behind the teeth and has a realistic location in the head. Wires which will generally fit are of length between $0 \mathrm{~mm}$ to $70 \mathrm{~mm}$. We investigated the wire in a homogeneous phantom with electromagnetic simulations and with thermal measurements on a real phantom. Resonance behavior of induced currents in the wire is difficult to visualize in the head model since the wire surroundings are highly heterogeneous. The SAR reducing effect of a wire spacer is also difficult to visualize in a consistent manner in the head model. For these reasons and due to the need for validation of the simulations we have constructed an homogeneous cylindrical phantom.

The choice of material in the phantom is chosen to be the worst case SAR scenario, it has the highest permittivity (relative $\epsilon=81$ ) of all tissues in the mouth with a wavelength of 55 $\mathrm{mm}$. This permittivity allows the full resonance length to be in the range of practical retainer wires.

The position and direction of the wire in the phantom influences the magnitude of the SAR hotspots around the wire. The direction of the wire in z-direction provides the highest conduction currents since the birdcage generates the major part of the $\boldsymbol{E}$ field is in this direction. However, the electric field around the two endrings of the birdcage has large components in xy direction compared to the fields in the rest of the birdcage. The wire direction in the phantom is chosen 
to be in the same orientation as it is for volunteers/patients, which is in the xy plane. This means that the xy-oriented wire will have a larger induced current when positioned near the end rings, which is a realistic situation and this could be a worst case positioning scenario.

We can conclude that the phantom simulations result versus the measurement results provide a slightly higher calculated $1 \mathrm{~g}$ SAR (maxima $33 \mathrm{~W} / \mathrm{kg}$ vs. $24 \mathrm{~W} / \mathrm{kg}$ ) but have similar temperature rise (maximum rise $1.4{ }^{\circ} \mathrm{C}$ after 15 minute scan) for the tested range of wires of 0 to $70 \mathrm{~mm}$. The difference in simulated and measured SAR could be explained by a number of factors. Probe positioning has been attempted to be as stable as possible, since reports on a small shift from the wire could lead to a large drop in measured temperature as noted by Bassen et al. [30]. It is a possibility that the real sensors are slightly further from the wire than they are in the simulations. It could also be that the accuracy of the sensor, or its thermal inertia is worse than the ideal sensor in simulations. However, a slight overestimation of the SAR in the simulations can in the case of safety prediction only be a positive remark. The input power to the measurements and simulations is 5 times higher than the scanner-indicated safety level for a normal head-scan. The maximum simulated $\mathrm{SAR}_{1 \mathrm{~g}}$ (calculated) of $32 \mathrm{~W} / \mathrm{kg}$ is for a normal scan $6.2 \mathrm{~W} / \mathrm{kg}$ which is just below the 5 minute $8 \mathrm{~W} / \mathrm{kg}$ IEC $1 \mathrm{~g}$ SAR RF safety limit.

The temperature measurements are performed to validate the simulations. We can conclude that both sets agree well and that the wire simulations therefore can be relied on. Additional simulations on the effectiveness of the spacer are performed to assess the influence of the spacer thickness. The final goal is assessing the safety of the wire in the head. To achieve this, we continue with simulations of wires bended along the curvature of the teeth, instead of straight wires and concluding with a head model simulation set in chapter 6 . 


\section{Chapter 6}

\section{EM simulations of phantom and head model in MRI birdcage}

The SAR in the tissue of a head model in a 7T head coil and the SAR in a cylindrical phantom are simulated and compared in this chapter using numerical electromagnetic analysis. A temperature measurement setup with the cylindrical phantom equal to the simulation setup has been used to compare and validate the phantom simulations, as described in the previous chapter. These simulations are proven to be valid, in this chapter we continue with this simulation setup. The investigation of a spacer around the wire, as briefly assessed in the previous chapter, is extended by determining the influence of spacer thickness, electrical conductivity and permittivity to SAR reduction.

\subsection{Electromagnetic simulation methods}

All of the described electromagnetic simulations are performed at $298.2 \mathrm{MHz}$, using either of the two finite-difference time-domain (FDTD) solvers Remcom xFDTD or CST microwave studio. An FDTD solver is selected for it is able to handle voxelized models and the combination of metal and dielectrics well. A 16-leg high pass birdcage head coil with length $180 \mathrm{~mm}$, diameter $300 \mathrm{~mm}$, is used driven by 32 discrete sources as depicted in Fig. 6.1
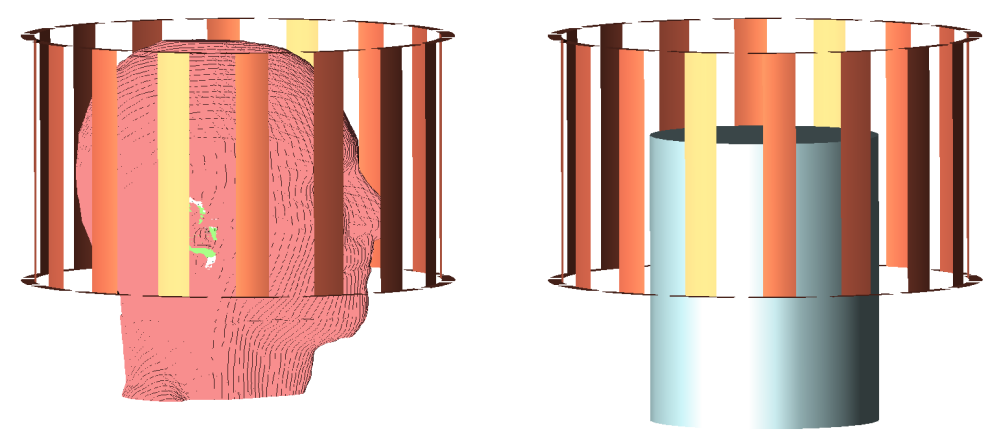

Figure 6.1: Head model of Duke[8] and the homogeneous cylindrical phantom. Both in a 7T birdcage head coil. 
In the figure we see the 16 vertical birdcage legs ended on both sides with an end ring. The sources are feeding in between every segment on the endrings, up to a total of 32 feeds. These are driven simultaneously on $298.2 \mathrm{MHz}$ with a phase difference of $360 / 16=22.5^{\circ}$ between consecutive sources on the rings to create a rotating $\boldsymbol{B}_{1}$ field. During simulations the $\boldsymbol{E}$ field and related voxel-based local SAR and SAR $1 g$ values are acquired together with the $\boldsymbol{H}$ fields and related $\mathrm{B}_{1}^{+}$and $\mathrm{B}_{1}^{-}$.

The described simulation setup enables the investigation of the RF safety of the retainer wire behind the teeth. To which the following section is intended as a guide to the steps taken in the additional simulation study. In short the following SAR simulations are performed, which are documented in Section 6.2

- Phantom: straight wire, thickness sweep of spacer

- Cylinder: straight wire, spacer $\sigma$ and $\epsilon$ sweep

- Phantom: bended wire vs straight wire with/without spacer

- Head model: bended wire with/without spacer

A curved stainless steel $0.55 \mathrm{~mm}$ diameter wire bended along the inner curvature of the teeth is chosen as standard wire in the head model. Two wires shapes are tested in the cylindrical phantom, the bended wire and the straight wire which has been verified in previous measurements. The SAR around both wires is compared with and without a spacer. When the straight and bended wire have a similar SAR distribution than the conclusions on resonance and on the spacer can be extended to the bended wire for the head model.

A $0.5 \mathrm{~mm}$ resolution head model of Duke in Fig. 6.6 is provided through the ITIS Virtual Family [8] is used for SAR simulation, Fig. 6.7. The head model is slightly modified such that the tongue is touching the wire in one case and not touching in the other case. Similar to the air gap, in Fig. 6.5a, around the wire in the phantom simulations. 


\subsection{Phantom simulation results}

\subsubsection{SAR reduction and spacer thickness}

The spacer around the wire has been proven to reduce SAR around a wire to background SAR (same as having no wire in place). That particular setup consisted of a $47.5 \mathrm{~mm}$ wire with a $0.5 \mathrm{~mm}$ layer of heat shrink, extending over the tips. In this section we investigate the relation between spacer thickness and SAR reduction. The layer of spacing around the wire is chosen in the range of 0 to $0.36 \mathrm{~mm}$. As a side note, these simulations require a very fine mesh to accommodate for the thin layers of air. Meshing the total simulation volume would result in a simulation time of weeks on a GPU accelerated workstation. Therefore the meshing at 0.2 $\mathrm{mm}$ is performed at a rectangular box only around the wire. The SAR results are shown next, including the phantom without a wire for comparison to what an effective spacing layer should achieve.
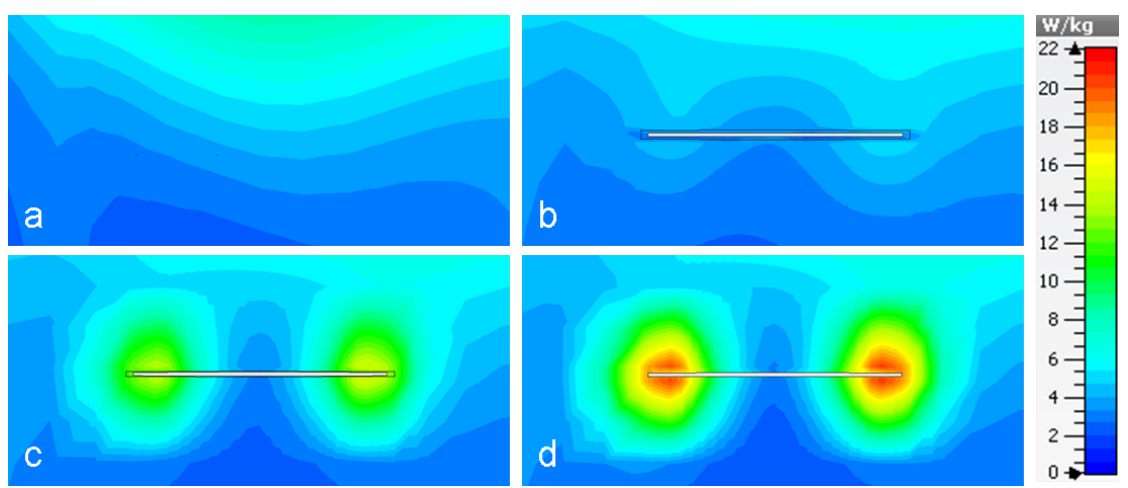

Figure 6.2: $1 \mathrm{~g}$ SAR for No wire(a), Wire with $0.36 \mathrm{~mm}$ separation layer (b), Wire with $0.16 \mathrm{~mm}$ separation layer (c) and wire no separation layer(d).

The spacer of $0.36 \mathrm{~mm}$ air around the wire reduces the bare wire $\mathrm{SAR}_{1 g}$ at the wire tip by a factor of 3.5, which is almost equal to the $\mathrm{SAR}_{1 g}$ at the same position without a wire. It appears that only a thin layer of non conductive spacer is needed to remove the added SAR contribution of the wire.

Table 6.1: E fields and SAR $1 \mathrm{~g}$ straight wire with spacer thickness sweep at location of maximum SAR for input power equal to whole phantom SAR $3.2 \mathrm{~W} / \mathrm{kg}$

\begin{tabular}{l|l|l} 
& \% of maximum local SAR & SAR $_{1 g}[\mathrm{~W} / \mathrm{kg}]$ \\
\hline \hline Only wire & $100 \%$ & 13 \\
No wire & $21.3 \%$ & 2.77 \\
\hline Air layer $0.13 \mathrm{~mm}$ & $64 \%$ & 8 \\
Air layer $0.36 \mathrm{~mm}$ & $29 \%$ & 3.7
\end{tabular}

The air layer is effective in not only blocking the conduction currents from flowing from tissue towards the wire and vice versa, but also in retaining the fields within the spacer volume as described in section 4.4. The normal component to the wire of the electric flux density $\boldsymbol{D}$ is continuous over the tissue-spacer boundary. From this boundary condition we calculate, as in Eq. (4.16), that the ratio of the normal components of the electric field at both sides of the 
boundary are in the order of 200 . The y-component of the electric field is normal to the tissuespacer boundary, as depicted in Fig. 6.3 and shows a much larger electric field in the spacer than the field in the tissue.

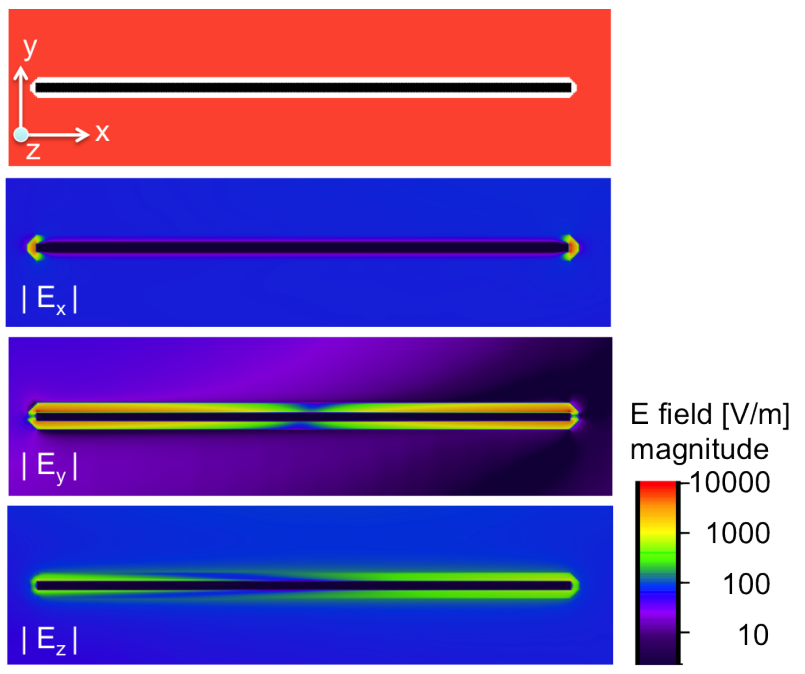

Figure 6.3: Wire with spacer. Electric field separated in $\mathrm{x}, \mathrm{y}$ and $\mathrm{z}$ components. xy-plane

The total electric field in the spacer is due to the normal components much higher than the measured field in surrounding tissue. The electric fields are stored in the volume of the spacer, rather than dissipated in the tissue. No dissipation or heating will take place in the spacer, since the spacer conductivity is zero.

\subsubsection{Spacer material selection}

If the proposed spacer is to be used in reality, a suitable material has to be selected. Since most of the practical materials that are biologically safe for use in the mouth are of low conductivity rather than non-conductive, we investigate the effectiveness of a set of practical low-conductivity materials. Several practical spacer materials, all $1 \mathrm{~mm}$ in diameter have been simulated of which the resulting E field in the tissue at $2 \mathrm{~mm}$ from the spacer tip is collected. The selected low conductivity materials are bee wax and air while for comparison teeth, saliva and muscle are added as 'spacer materials'. The material properties are outlined in table 4.2. Further more a sweep of permittivity and conductivity of the spacer material is performed to check their relation to the SAR. The phantom cylinder used in this particular simulation setup is chosen to have the electrical properties of muscle, which has a slightly larger wavelength than the HEC-gel. 


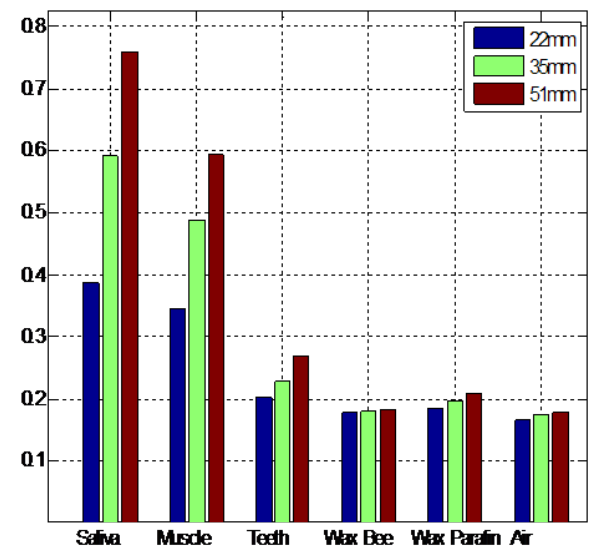

(a) several practical spacers, wire lengths $22 \mathrm{~mm}$, $35 \mathrm{~mm}$ and $51 \mathrm{~mm}$

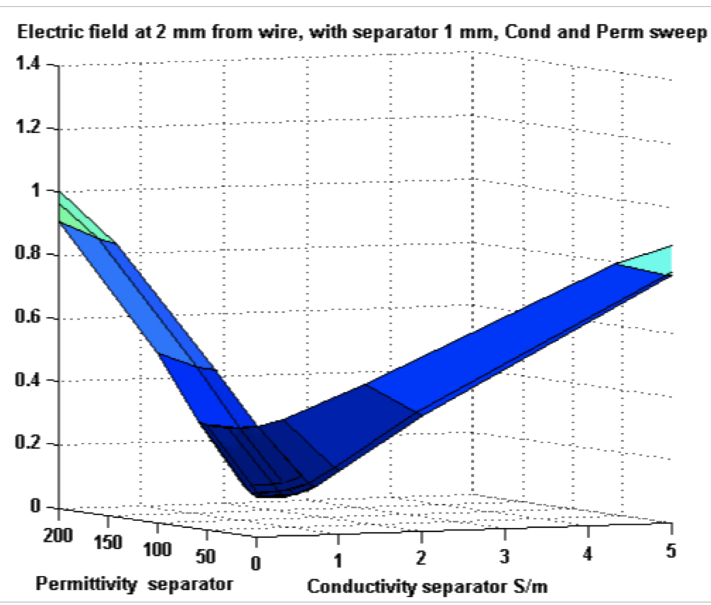

(b) Spacer conductivity and permittivity sweep

Figure 6.4: Electric fields $2 \mathrm{~mm}$ from the $1 \mathrm{~mm}$ spacing, at the wire tip

The E fields are found to be lowest for a non conductive spacer, such as air. A minimally conductive spacer for example Bee wax $\left(\sigma\right.$ is $\left.10^{-12} \mathrm{~S} / \mathrm{m}\right)$ performs as well as air. Both of these spacers fully reduced the increase in SAR as if the wire is not present. The higher conductivity materials muscle $(\sigma$ is $0.77 \mathrm{~S} / \mathrm{m})$ and Saliva $(\sigma$ is $0.34 \mathrm{~S} / \mathrm{m})$ show the same SAR as the bare wire case or even worse. The exact electric field results for the practical spacer materials are given in the following table

Table 6.2: E fields at $2 \mathrm{~mm}$ axial distance from the spacer tip, relative input power: the ratio between $\mathrm{E}$ field measurements is the important quantitiy here, not the field strength itself. For $35 \mathrm{~mm}$ wire straight wire with spacer material variety

\begin{tabular}{l|ll} 
& \% of maximum Efield & Efield $[\mathrm{V} / \mathrm{m}]$ \\
\hline \hline Only wire & $\mathbf{1 0 0} \%$ & 0.48 \\
No wire & $37.5 \%$ & 0.18 \\
\hline Saliva & $123 \%$ & 0.59 \\
Tongue (muscle) & $100 \%$ & 0.48 \\
Teeth & $45.8 \%$ & 0.22 \\
Paraffin wax & $42.0 \%$ & 0.20 \\
Bee Wax & $37.5 \%$ & 0.18 \\
Air & $37.5 \%$ & 0.18
\end{tabular}

The first data column in the table allows for comparison of the field magnitude for different spacers around the wire in muscle. The saliva spacer makes the electric fields worse compared to having the wire in muscle and with it the SAR . Similar data is found in the parameter sweeps of Fig. 6.4b, in which the simulated electric field rises with increasing conductivity or permittivity. A conductive spacer allows conduction currents to flow in and out of the wire straight in the tissue especially at the tips, creating a higher SAR . This effect is discussed earlier in Section 4.4.2. Bee wax, silicone rubber, or an equivalent low conductivity and low permittivity material would be very suitable for use as spacer. These materials for $1 \mathrm{~mm}$ thickness all reduce the SAR to the level of having no wire at all. Teeth as a spacer are not to bad either, since a hypothetical 
$1 \mathrm{~mm}$ layer of teeth reduces the electric fields to $45 \%$ of the bare wire maximum, and therefore the SAR to $20 \%$ of the bare wire maximum.

\subsubsection{Phantom simulations bended wire vs straight wire}

In this section the difference in SAR of the previously assessed straight wire and the bended wire for the head model is investigated. The influence of a spacer around both wires is also simulated to compare its effectiveness to a spacing around the wire in the head model.

A comparison of the difference in SAR around both wires (bended and straight) can be made by thinking of the retainer wire and straight wire as a transmission lines. Hypothetical distributed sources along the wire lengths are fed by the incident field [14]. Changing the shape will alter the phase distribution of the incident field over the wire and as a result change the magnitude of the current over the transmission line.

The phase front in the cylinder is fairly flat due to the symmetrical shape of both cylinder and bird cage coil. Moreover since the change in shape between bended and straight wire is small compared to the wavelength, we expect the phases of the hypothetical distributed sources to be the same between bended and straight wire. The difference in induced current is therefore small which in turn results in a small change in SAR. The results of the simulations of local SAR are illustrated in the following figures. Additionally, the bare wires are in a second setup surrounded by a non-conductive (air) spacer.
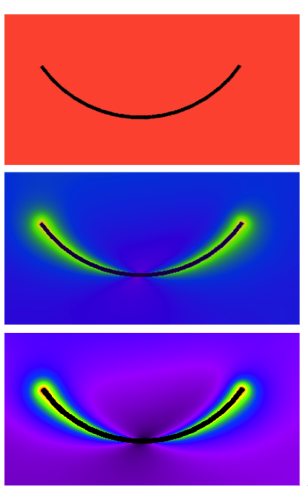

(a) Bended: bare wire(left) and wire with spacer (right)
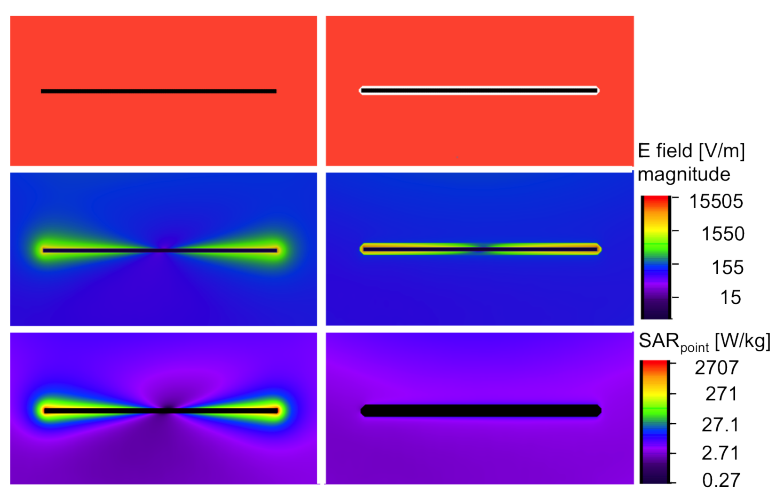

(b) Straight: bare wire(left) and wire with spacer (right)

Figure 6.5: Bended and straight wires in a homogeneous phantom, with and without $3 \mathrm{~mm}$ spacer. Electric field and SAR results

The simulation results of the bended wire and straight wire (Fig. 6.5a and Fig. 6.5b) provide local SAR values of almost the same distribution over the length of the wire. The SAR values at the position of maximum SAR around the wire are given in table 6.3. As a result of the SAR values of the straight and bended wires being almost identical we can use the bended wire for further investigations in the head model. 
Table 6.3: Local SAR and 1 gram SAR for a $32 \mathrm{~mm}$ bended wire and a straight wire in phantom. Measured at the location with maximum SAR, Input power: $38.7 \mathrm{~W}$

\begin{tabular}{l|l|l|l} 
& $\begin{array}{l}\text { \% of maximum } \\
\mathrm{SAR}_{1 \mathrm{~g}}\end{array}$ & local SAR $[\mathrm{W} / \mathrm{kg}]$ & $\mathrm{SAR}_{1 \mathrm{~g}}[\mathrm{~W} / \mathrm{kg}]$ \\
\hline \hline Bended wire & $91.7 \%$ & 2100 & 7.8 \\
Straight wire & $100 \%$ & 2707 & 8.5 \\
No wire & $61.2 \%$ & 9 & 5.2 \\
\hline Bended wire spacer & $61.2 \%$ & 9 & 5.2 \\
Straight wire spacer & $61.2 \%$ & 9 & 5.2
\end{tabular}

The local SAR for the wire with spacer is equal to having no wire at all. This is a good result for RF safety; the spacer fully neutralizes the addition of any SAR by the wire. The 1-gram SAR is showing a more moderate behavior compared to the very high local (mg) SAR, explainable because the high levels of energy absorption is only taking place in a small volume around the wire. Comparing the two wire shapes: The wires both introduce a 1-gram SAR about twice as high as the background SAR (phantom without a wire). Their maximum values differ only 0.7 $\mathrm{W} / \mathrm{kg}(9 \%)$, for which the bended wire has slightly lower SAR. We can summarize that the wires behave practically the same in terms of SAR which enables us to apply the the conclusions of the previous straight wire study to the bended wire of same length.

\subsection{Head model simulations}

The head model of Duke in Fig. 6.6 is provided through the ITIS Virtual Family [8], with a high resolution of $0.5 \mathrm{~mm}$ and is used for SAR simulation in $\mathrm{xFDTD}$.
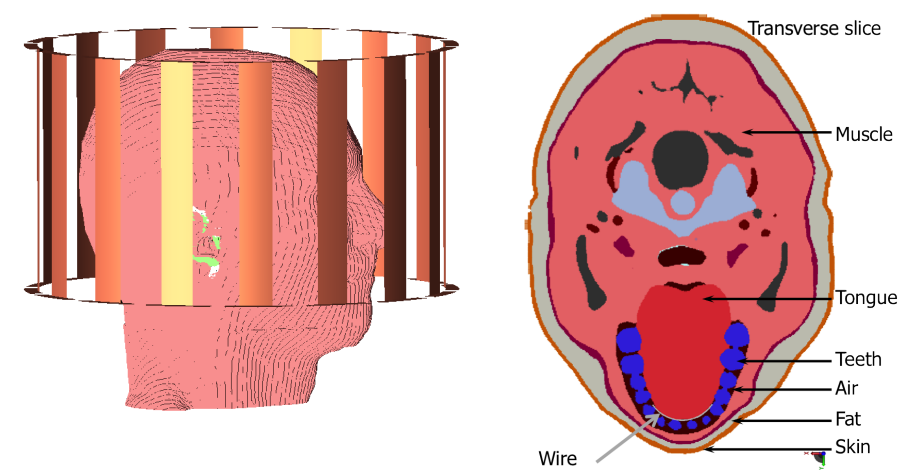

Figure 6.6: Head model Duke in birdcage with wire. Transverse slice of the head with the curved wire

The retainer wire used in the head model setup is $32 \mathrm{~mm}$ in length, has an electrical conductivity of $5 \cdot 10^{5} \mathrm{~S} / \mathrm{m}$ and follows the curve of the upper set of teeth. Two closeup views of the wire section in the mouth are depicted in figure 6.7. For the first scenario, the tongue is in contact with one side of the wire over the full length, which is termed the 'embedded case'. The other side of the wire is mainly in contact with air and at points with the teeth. In the second scenario, in which the wire and tissue are separated, the segment of tongue close to the wire is replaced with air up to a distance of $3 \mathrm{~mm}$ from the wire. This is approximately the same thickness as a 
for a sports mouthguard. The wire is now only in contact with air and the teeth. The following simulation results are summarized in Tables 6.4 and 6.5
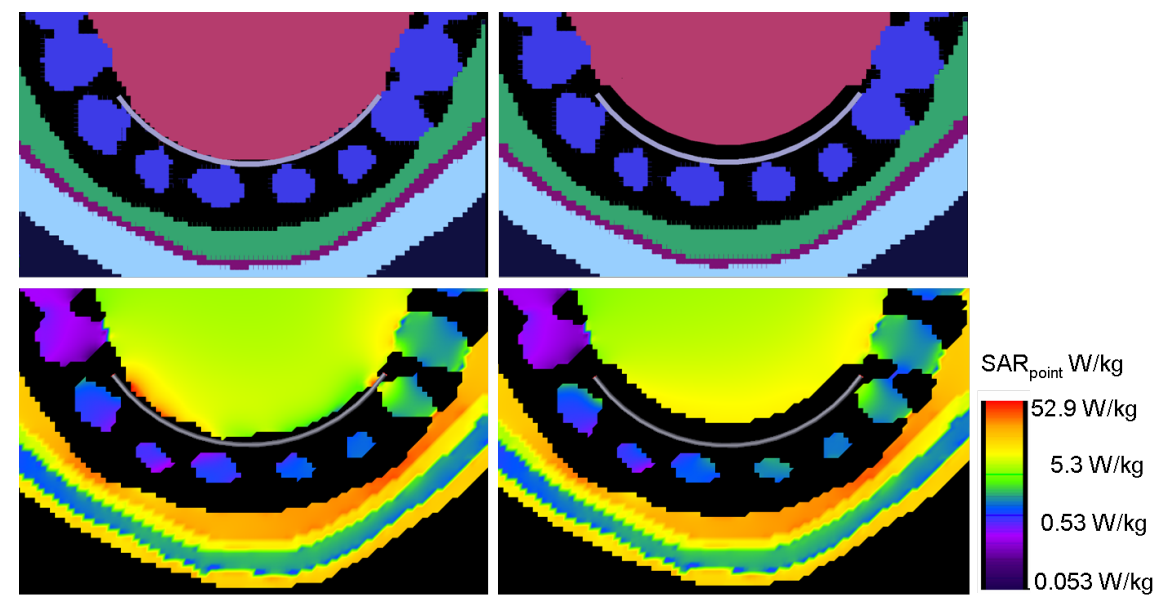

Figure 6.7: Head model, simulated local (mg) SAR around the $35 \mathrm{~mm}$ wire. Bare wire against the tongue (left) and without contact (right)

The local $0 \mathrm{~g}$ SAR, 1 gram SAR and 10 gram SAR maxima in the head and around the wire are given in table 6.4. The SAR values at the maximum local SAR spot next to the wire are given in table 6.5. The local SAR is highest around the wire in the transverse head-slice at the position of the wire. The 1 gram and 10 gram SAR maxima however, are located somewhere else in this slice and are not influenced by the presence of the wire. The overall head model maximum 1-gram SAR is located at the top of the head. This region is close the the top end-ring of the birdcage coil which introduces a higher dissipated power compared to regions further away from the end rings.

Table 6.4: Head model with $32 \mathrm{~mm}$ bended wire for simulation $\mathrm{P}_{\text {input }}=38.7 \mathrm{~W}$. Local SAR, 1-gram and 10-gram SAR averages

\begin{tabular}{l|l|l|l} 
Location & local SAR $[\mathrm{W} / \mathrm{kg}]$ & $\mathrm{SAR}_{1 \mathrm{~g}}[\mathrm{~W} / \mathrm{kg}]$ & $\mathrm{SAR}_{10 \mathrm{~g}}$ \\
\hline Head maximum SAR & 253 & 64.2 & 21.9 \\
Wire slice maximum SAR & 52.9 & 17.7 & 14.1 \\
Wire slice no-wire maximum SAR & 36.37 & 17.7 & 14.1
\end{tabular}

Table 6.5: Head model with $32 \mathrm{~mm}$ bended wire for simulation $\mathrm{P}_{\text {input }}=38.7 \mathrm{~W}$. Local SAR, 1-gram and 10-gram SAR averages

\begin{tabular}{l|l|l|l} 
Location of $\max \mathrm{SAR}_{0 \mathrm{~g}}$ around wire & local SAR $[\mathrm{W} / \mathrm{kg}]$ & $\mathrm{SAR}_{1 \mathrm{~g}}[\mathrm{~W} / \mathrm{kg}]$ & $\mathrm{SAR}_{10 \mathrm{~g}}$ \\
\hline No wire & 3.39 & 5.62 & 5.77 \\
With wire & 52.9 & 6.39 & 5.83
\end{tabular}

The addition of the $3 \mathrm{~mm}$ spacer shows a reduction in the 1 gram SAR when the tongue is not touching the wire, reducing it to the level of having no wire at all. These head-model simulations show that a reduction in SAR around the wire in the head is possible. Although for this typical length of wire any reduction does not appear to be necessary since the maximum 1 gram SAR is not found around the wire, but elsewhere in the top volume of the head. 
A general remark on the SAR distributions of the head simulations. The head simulations show an elevated SAR level on the left side of the head, in the tongue as well as in the lip, compared to the right side. This difference is not observed in the cylindrical phantom as described in the following sections, which displays a concentric distribution of SAR . Interaction of the non cylindrical shape of the head with the sources of the birdcage which are creating a clockwise circularly polarized field is most probably the cause for the uneven SAR distribution. A reversed direction of the field rotation, into counter clockwise, reveals a mirrored SAR distribution in the slice.

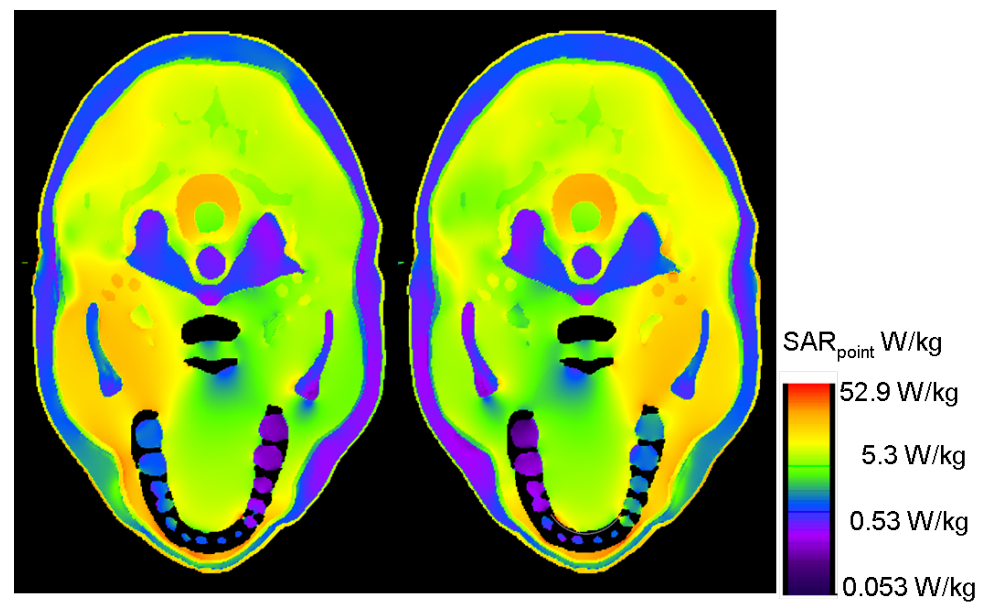

Figure 6.8: Head model $1 \mathrm{~mm}$ voxel resolution, local SAR, clockwise and counter clockwise RF excitation

The same head model simulation setup with reversed circular polarity of the rotating field shows mirrored SAR hotspots on the sides of the head.

\subsection{Simulation data discussion and preliminary conclusions}

The wire in the head model is positioned behind the teeth of the upper jaw and has a typical retainer wire length of $32 \mathrm{~mm}$. The head model of Section 6.3 is positioned in the birdcage head coil as is done in a clinical setup, in which the wire is now closely located to the lowest end ring of the birdcage. The areas around the end rings are known to have high E fields and high SAR in the surrounding tissue. The phantom simulations are performed to investigate the difference in SAR due to the presence of a spacer and to compare the bended wire of the head model to a similar wire with a straight shape. The z-position of the wire in the phantom is at equal to the head model, such that it is at the same position with respect to the birdcage end ring.

The local SAR as defined in a very small volume of one voxel in the simulation provides a good measure to pinpoint possible temperature hotspots. However due to spatial averaging behavior of a SAR hotspot in terms of resulting temperature distribution, the choice for local SAR is not the best measure for safety assessment, since a small hotspot will average out over a larger region. Common in safety regulations is the 1 gram average or 10 gram average SAR. The head model simulations show a definite increase in local $(\mathrm{mg})$ SAR $(52.9 \mathrm{~W} / \mathrm{kg}$ vs. $3.4 \mathrm{~W} / \mathrm{kg})$ when the wire is added to the teeth, however comparing to $1 \mathrm{~g}$ averaged values, this increase is far less significant - if seen at all. The 10 gram average around the wire does not show any increase 
around the wire at all. The phantom simulations on the other hand, do indicate an increase in $1 \mathrm{~g}$ SAR around the wire. This could be explained by the resonance behavior. The wavelength in the phantom is always smaller or equal to the wavelength in the head, therefore, every wire in range of 0 to $55 \mathrm{~mm}$ will therefore be closer to resonance in the phantom and have a higher SAR .

As it appears from the head simulations, the $32 \mathrm{~mm}$ wire does not have the largest contribution to the 1 gram or 10 gram averaged SAR in the head. It could be argued that due to this fact, this wire can be rated MR -compatible. However worst case scenario's are difficult to predict for the head model. Moreover it is noted that during the related phantom simulations, we do see an increase in the $1 \mathrm{~g}$ SAR averages leading to a temperature rise.

Since the SAR behavior in the head model is not completely predictable and could have a SAR higher than the regulations prescribe, we investigated the use of a spacer which is lowering the SAR around the wire. A spacer layer of $3 \mathrm{~mm}$ around the wire effectively canceled the increase in local SAR in the head model, as seen in Section 6.3. The most effective spacer is the non-conductive, low permittivity spacer, from Section 6.2.2. The spacer effectively isolates the conduction currents from flowing in and out of the wire. The phantom simulations showed that wire with a $0.38 \mathrm{~mm}$ layer of non conductive material has an increase in $1 \mathrm{~g}$ SAR compared to the background $1 \mathrm{~g}$ SAR by a factor of 1.4, while the same wire -bare- has a 3.5 fold increase in SAR, Section 6.2.1. A wire with a spacer of $1 \mathrm{~mm}$ layer thickness had no increase in SAR at all as seen in Section 6.2.2.

Concluding We have seen in the previous chapters that a bare wire at maximum resonance in a tissue mimicking phantom can have a 10 fold gain in SAR as measured by temperature sensors an reproduced in simulations. Shorter wires up to $65 \%$ of the resonance length have a 1 gram SAR in the range of background $\mathrm{SAR}_{1 \mathrm{~g}}$ up to a 5 fold increase which can be used in SAR safety assessments. The typical retainer wire length of $32 \mathrm{~mm}$ did not impose a 1 gram $\mathrm{SAR}$ level higher than the maximum $\mathrm{SAR}_{1 \mathrm{~g}}$ throughout the whole head. This wire length is therefore MR RF compatible. Longer wires are closer to resonance length and should to be safe be surrounded by a spacer.

When a thin spacing layer of $1 \mathrm{~mm}$ thickness fully surrounds the wire, the 1 gram SAR is dropped to background levels as is measured in the phantom setup, during simulations and measurements. If the insulated wire is directly connecting to the tissue through a leak at the wire tips, this could have local SAR even higher than the bare wire. As such, a spacer applied around retainer wire should be sturdy enough to keep the tongue, which is the most likely conductive tissue to touch the wire, at distance. A sports mouthguard would probably work well as is simulated for the head model in Section 6.3. The SAR in the teeth is considerably lower than the SAR present in the tongue. A spacer on the teeth side of the wire is therefore not necessary. 


\section{Chapter 7}

\section{Discussion and conclusions}

The safety of scanning a person with a dental retainer wire at a 7 tesla MR scanner is investigated. Metal wires can induce a high specific absorption rate (SAR) in tissue around them when being scanned in an MR machine as reported in literature [5], [6], [7]. These metal wires are commonly made of stainless steel or titanium and have a length between $20 \mathrm{~mm}$ and $70 \mathrm{~mm}$. Worst case scenario's could not be assessed only with a head model and practical validation on a head was not permissible. For these reasons we carried out simulations and temperature measurements on a phantom with tissue mimicking electrical properties in the 7 tesla scanner for validation purposes. The investigation is carried out with temperature measurements and simulations on the set of wires of $20 \mathrm{~mm}$ to $70 \mathrm{~mm}$ in a phantom. In which an FDTD numerical electromagnetic solver provided quantitative SAR results around the wires in the phantom. The measurements are in good agreement with the simulations. The head model, Duke, for electromagnetic simulations is provided by the ITIS foundation [8] in which we modeled a 32 $\mathrm{mm}$ retainer wire.

Tissue heating and SAR around a wire due to radio frequent (RF) electromagnetic fields are based on energy dissipation. Induced conduction currents by the incident field in tissue around the wire will channel towards the wire since this has a higher conductivity. A wire inside the electromagnetic RF field of a transmitting MR head coil will induce an electric current over the length of the wire. These conduction currents will flow in and out the wire, mainly via the the wire tip, creating a hotspot at that location. If the wire length is equal to approximately 0.5 times the wavelength of the incident field, then a resonating effect will take place. The current in resonance will increase and with it also the in and outflowing currents to the tissue. Since tissue is a conductive material, this current will partly be dissipated following the complex Poynting theorem of Eq. 2.18 and will heat the tissue to a certain extend. The IEC states that the temperature of tissue should not rise more than $1^{\circ} \mathrm{C}$.

\subsection{Discussion}

On the power settings. Temperature measurements on the tissue mimicking phantom were carried out in the head coil of the 7 tesla MR scanner initially at full permissible power. That is, the amount of power which is maximally allowed for a clinical head scan. This did not result in any significant heating around the wire, with a maximum temperature rise of only $0.3^{\circ} \mathrm{C}$ after 10 minutes continuously scanning. In order to be able to verify temperature rises around the scanner we had to overrule the SAR safety settings by a factor five $\left(\mathrm{P}_{\mathrm{avg}}=70 \mathrm{~W}\right)$, such that 
the SAR amplifying effect of the wire becomes measurable as a temperature rise. The power scaling between measurements and simulations could not be done one-to-one, since the power is not known directly at the feed points of the MR coil. Linking the $\mathrm{B}_{1}^{+}$transmit efficiency of the scanner to the transmit efficiency of the simulated head coil made the scaling of power possible as is described also in [26].

Resonance. A large influence on the SAR magnitude is the resonance length of the wire. A fully resonating wire has the highest SAR as well as the highest temperature rise. Our simulation and measurement results provide similar values for the temperature rise over a range of wires of 0 to $70 \mathrm{~mm}$, over a time period of 15 minute excitation which is the recommended time following the ASTM RF safety assement for passive implants [21]. The input power for the measurements and simulations is $70 \mathrm{~W}$ MR average power. The maximum increase is measured at $1.4^{\circ} \mathrm{C}$ for a resonant wire of $55 \mathrm{~mm}$ wire. Simulations of the same phantom and head coil at the same power have a maximum temperature increase of $1.5^{\circ} \mathrm{C}$ for a $47.5 \mathrm{~mm}$ wire. The temperature rise for the measurement without a wire was $0.8^{\circ} \mathrm{C}$ and for the simulation $0.6^{\circ} \mathrm{C}$. As such, the measurement and simulation results are in good accordance. It was noted that the measured and simulated values for the maximum $1 \mathrm{~g}$ SAR are slightly different for the resonant wires: The simulated $1 \mathrm{~g}$ SAR maximum for a $47.5 \mathrm{~mm}$ wire is $33 \mathrm{~W} / \mathrm{kg}$ vs. $24 \mathrm{~W} / \mathrm{kg}$ for a $55 \mathrm{~mm}$ wire in the measurement. The $1 \mathrm{~g}$ SAR without a wire measured and simulated are both $4 \mathrm{~W} / \mathrm{kg}$. The input power to the measurements and simulations is 5 times higher than the scanner-indicated safety level for a normal head-scan. The maximum simulated $\mathrm{SAR}_{1 \mathrm{~g}}$ value is $32 \mathrm{~W} / \mathrm{kg}$, which for a scan at normal powers equal to $6.2 \mathrm{~W} / \mathrm{kg}$. The 'normal scan' $\mathrm{SAR}_{1 \mathrm{~g}}$ value is just below the 5 minute $8 \mathrm{~W} / \mathrm{kg}$ IEC $1 \mathrm{~g}$ SAR RF safety limit. Tissues in the mouth close to the retainer wire all have lower or equal permittivity than the phantom material. As such in terms of resonance the mentioned temperatures are the worst case since the resonant wavelength of the other materials in the mouth is longer, leaving most common retainer wires in the safer region below resonance length. The measurements showed that wires shorter than $65 \%$ of the resonance length have an $1 \mathrm{~g}$-SAR of half the maximum SAR or less.

Separator Electrically isolating the wire from conduction currents in the tissue will have the greatest reduction on tissue heating as described in Sections [4.4] and [6.3]. A spacer is found most effective for a non-conductive spacer such as air, bee-wax or silicone. The use of a spacer has been investigated in the experimental setup as well in Section 5.3.1. A bare $47.5 \mathrm{~mm}$ wire registered a $\mathrm{SAR}_{1 \mathrm{~g}}$ of $33 \mathrm{~W} / \mathrm{kg}$ for $70 \mathrm{~W}$ MR average power. When a spacer with $0.38 \mathrm{~mm}$ layer thickness was applied as a result the $\mathrm{SAR}_{1 \mathrm{~g}}$ was reduced to $4 \mathrm{~W} / \mathrm{kg}$ with a maximum temperature rise of $0.9^{\circ} \mathrm{C}$ after 15 minutes of scanning. The reduced values are almost the same as the background SAR of $3.2 \mathrm{~W} / \mathrm{kg}$ and temperature rise of $0.8^{\circ} \mathrm{C}$ after 15 minutes. The $1 \mathrm{~g}$ SAR around the wire was reduced by a factor of 8 . Additional simulations of a $1 \mathrm{~mm}$ air spacer show complete neutralization of the SAR around the wire, even for the resonant wires having the highest SAR. It has been noted in literature that an isolated wire with a bare metal tip, such as a guide wire in interventional MRI can quickly become very hot [19] at the bare tip. Since conduction currents can only flow in and out at that point, this potentially dangerous behavior can occur. The spacer around the wire should for this reason keep any conductive tissue, such as the tongue, away from the wire. In addition, the teeth have a natural layer of insulation to the conduction currents and will not show an increased SAR when in contact with a metal wire. 
Head simulations. Simulations on the head with retainer wire, with and without separation are performed. A $52.9 \mathrm{~W} / \mathrm{kg}$ increase in local $(\mathrm{mg}) \mathrm{SAR}$ is seen in the head model simulations for a typical retainer wire of $32 \mathrm{~mm}$, however the 1 gram averaged SAR does not show an increase in SAR when the wire is inserted. The local $(\mathrm{mg}) \mathrm{SAR}$ is of no significance if only a small volume has high SAR, [30]. A very high mg SAR only occurs at the tip edge of the wire, where the field is high but drops off quickly in the tissue. Since a temperature rise is averaged out over a larger area, a small volume of high SAR will have no impact on the safety of the tissue. As it appears from the head simulations, the $32 \mathrm{~mm}$ wire is not creating the highest $1 \mathrm{~g}$ or $10 \mathrm{~g}$ averaged SAR in the head, the maximum is created in the top part of the head. Due to the fact that the wire is not creating the highest SAR, this wire can be rated MR -compatible. Patients or volunteers with such a wire of this length or shorter should be able to safely have an head scan in terms of SAR . For longer wires closer to resonance we have investigated a spacer in the head model. The maximum local (mg) SAR around the $32 \mathrm{~mm}$ bare retainer wire in head model Duke [8] was registered at $52.9 \mathrm{~W} / \mathrm{kg}$ during simulations and is reduced to the level of not having a wire at all $(4 \mathrm{~W} / \mathrm{kg}$ ) when a $3 \mathrm{~mm}$ spacer was applied. Confidence for this spacer thickness is given since even the local (mg) SAR was reduced to background values. Longer wires with lengths closer to resonance will also be safe to use when such spacer is applied.

\subsection{Concluding}

The safety of a dental retainer wire in terms of SAR in tissue surrounding the wire has been investigated. The SAR limits by the IEC for any 1 gram of tissue in the head due to RF excitation is $8 \mathrm{~W} / \mathrm{kg}$ during a period of 5 minutes and secondly the whole head averaged SAR should be below $3.2 \mathrm{~W} / \mathrm{kg}$. This study provided results to electromagnetic head model simulations, at 298.2 MHz (7T), in which a retainer wire of common wire length $(32 \mathrm{~mm})$ did not increase the maximum 1 gram average SAR compared to not having a wire. Due to this fact, we can say that people with one wire up to $32 \mathrm{~mm}$ can be admitted for a 7 tesla head scan.

The power dissipation due to longer wires up to resonance length could pose a risk for conductive tissue touching the retainer wire. Measurements on wires in the range of 0 to $70 \mathrm{~mm}$ in a saline gel phantom showed maximally a ten fold increase in $1 \mathrm{~g}$ SAR for a resonant wire. This particular resonant wire of $56 \mathrm{~mm}$ in length had a $1 \mathrm{~g} \mathrm{SAR}$ of $6.2 \mathrm{~W} / \mathrm{kg}$ during a normal head scan at $14 \mathrm{~W}$ average power, these values are scaled from the actual scan at $70 \mathrm{~W}$ scan resulting in a calculated 1 gram SAR of $32 \mathrm{~W} / \mathrm{kg}$. A wire is as a rule of thumb at resonance when the RF wavelength of the excitation field is equal to half the wire length. At 7 tesla the resonance length of a wire is due to the surrounding media theoretically $55 \mathrm{~mm}$ in saliva (equal to the phantom wavelength), $61 \mathrm{~mm}$ in muscle, $132 \mathrm{~mm}$ in teeth and $500 \mathrm{~mm}$ in air. The wavelength of the field around the retainer wire is in practice a weighted average of the aforementioned lengths. Wires up to $65 \%$ of the resonant length maximally have maximally a five fold $1 \mathrm{~g}$ SAR increase, as was measured and simulated in the phantom study. Wires between the shortest resonance condition of $55 \mathrm{~mm}$ and the maximum retainer length of $70 \mathrm{~mm}$ will most likely not become fully resonant in the head due to the predominant combination of teeth and air and could possibly be issued MR compatible for that reason. However resonant, not safe, scenarios could occur in the head for longer wires and for this reason we investigated a SAR reducing spacer.

We investigated the use of an electrically non-conductive spacer around the wire to lower the SAR with the goal of enhancing the RF safety. Such a spacer blocks conduction currents which are induced in the tissue and spacer by the incident field of the MR head coil. The conduction currents flow from the tissue through the wire and vice versa and cause an SAR hotspot around 
the wire tips. A spacer layer of $0.38 \mathrm{~mm}$ thickness reduced the 1 gram SAR of a resonant wire by a factor of 8 , as was simulated and validated through phantom temperature measurements. The head model with a $3 \mathrm{~mm}$ spacing between tongue and wire even had no local SAR increase compared to the head model without wire. A practical solution would be to use a silicone sports mouthguard. Such a spacer, which will have to fully cover the wire together with the teeth, makes scanning of people with a longer retainer wire RF safe as well.

The conclusion of this study for the safety of retainer wires is summarized in two statements. Short wires up to $32 \mathrm{~mm}$ can be considered RF safe for a 7 tesla MR head scan. Longer wires are not always RF safe. A solution to longer wires is to fully cover the wire with a nonconductive spacer such as a silicone sports mouthguard to ensure RF safety. As a consequence of these outcomes, people with a retainer wire have the possibility of going into the 7 tesla MR scanner.

Recommendations In this investigation on the safety of retainer wires we have looked at single wires in the range of 0 to $70 \mathrm{~mm}$ in length. Further research on retainer wires could be the following two topologies. A retainer wire snapped in two, who's halves are reconnected by tissue, is thought of being a hazard. It is possible that a safe wire as a whole is not resonating, but that the change through the cut would make this wire resonant and unsafe. A second topology which is not uncommon involves having two retainer wires behind the teeth. The interaction of the fields with these wires might lead to resonance on a larger scale. These two topics were however not the scope of this thesis. The time needed to investigate multiple two-wire scenarios, using simulations and experiments would be too long for one thesis. 


\section{Bibliography}

[1] Schuller AA, Poorterman JHG, Kempen CPF van, Dusseldorp EML, Dommelen P van, Verrips GHW. Kies voor Tanden. Een onderzoek naar mondgezondheid en preventief tandheelkundig gedrag van jeugdigen. TNO, Leiden, 2011

[2] MRI hardware setup. Kristen Coyne MRI: A Guided Tour. National High Magnetic Field Laboratory. Web. http://www.magnet.fsu.edu/education/tutorials/java/mri/index.html

[3] Image of head scan. Yorkshire center for health informatics - Leeds (YCHI Leeds) www.ychi.leeds.ac.uk/radinfo/MRI/MRIHead/tabid/59/Default.aspx 26062008

[4] Neil V. Budko, Electromagnetic Radiation, Scattering and Imaging. Lecture notes 2004

[5] Konings MK, Bartels LW, Smits HFM, Bakker CJG, Heating around intra vascular guide wires by resonating RF waves. J Magn Reson Imaging 2000;12:79.

[6] Nitz WG, Oppelt A, Renz W, Manke C, Lenhart M, Link J, On the heating of linear conductive structures as guidewires and catheters in interventional MRI. J Magn Reson Imaging 2001.

[7] Yeung CJ, Susil RC, Atalar E, RF Safety of wires in interventional MRI. Using a safety index J Magn Reson Imaging 2002;47;187-193

[8] ITIS Foundation, Virtual family, Zurich

[9] Gabriel et al. University Florence, body tissue dielectric properties for a wide range of frequencies

[10] M. Haacke, R. Brown, M. Thompson, R. Venkatesan, Magnetic Resonance Imaging, Physical Principles and Sequence Design.

[11] Chew WC, Waves and fields in inhomogeneous media, IEEE Press Series on Electromagnetic Wave Theory. February 1999

[12] Balanis, Advanced Engineering Electromagnetics, John Wiley and Sons, 1989

[13] Verweij, M.D., van den Berg, P.M., Blok, H. Electromagnetic waves, an introductory course Published on behalf of the vssd 2001

[14] Acikel V, Atalar E, Modeling of radio-frequency induced currents on lead wires during MR imaging using a modified transmission line method. Med. Phys 38(12), December 2011

[15] R.C. Weast, Handbook of Chemistry and Physics. Edition 53 Cleveland, Ohio, 1972.

[16] R. de Graaf, In Vivo NMRSpectroscopy: Principles and techniques John Wiley \& Sons, Chichester, UK, 1999 
[17] Baker, K. B., Tkach, J. A., Phillips, M., Rezai, A. R., Variation in RF-induced Heating of a Deep Brain Stimulation Implant Across MR Systems Journal of Magnetic Resonance Imaging, Vol 24, 2006, p. 1236.

[18] Lier van, A., Brunner, D. O., Pruessmann, K. P., Klomp, D.W.J., Luijten, P.R., Lagendijk, J.J.W, and Berg van den, C.A.T. $B_{1}^{+}$Phase mapping at $7 T$ and its application for in vivo electrical conductivity mapping Magn. Reson. Med 2012

[19] Nyenhuis ISMRM 2008 syllabus implants

[20] Nyenhuis, J.A., Park, S.M., Kamondetdacha, R. Amjad, A., Shellock, F.G., Rezai, A.R. MRI and Implanted Medical Devices: Basic Interactions With an Emphasis on Heating IEEE transactions on device and materials reliability. Vol.5, No.3, 2005

[21] ASTM F2182-02a. Standard test method for Measurement of radio frequency induced heating near passive implants during magnetic resonance imaging

[22] ASTM F2052 - 06. Standard test method for Measurement of magnetically induced displacement force on medical devices in the magnetic resonance environment

[23] Lorentz, H.A. The theorem of Poynting concerning the energy in the electromagnetic field and two general propositions concerning the propagation of light Versl. K. Akad. Wet. Amsterdam. Wis. en nat. afd., 41896

[24] Ibrahim TS. Analytical approach to the MR signal Magn. Reson med. 2005

[25] Yarnykh. V.L. Actual Flip-Angle imaging in the pulsed steady state: A method for rapid three-dimensional mapping of the transmitted Radiofrequency field Magn. Res. in Med. 2007

[26] Teeuwisse W.M., Brink. W.M, Webb A.G. Quantiative assessment of the effects of high permittivity pads in 7 tesla MRI of the brain Magn. Res. in Med. 2011

[27] Instructive introductory MRI course material www.imaios.com/en/e-Courses/e-MRI

[28] Epstein, C.L., Introduction to the mathematics of medical imaging

[29] Material specifications from: Gabriels list Italian national research council, Wolfram alfa, silicone.jp, asiinstr.com.

[30] Bassen, H., Kainz, W., Mendoza, G., Kellom, T, MRI-induced heating of selected thin wire metallic implants -laboratory and computational studies - findings and new questions raised Minimally Invsive Therapy. 15;2; 76-84; 2006

[31] Shellock F.G., Woods, T.O., Crues J.V., MR labeling information for implants and devices: explanation of terminology. Radiology 2009; 253(1):26-30 